\title{
Directed migration of microscale swimmers by an array of shaped obstacles: modeling and shape optimization
}

\author{
Jiajun Tong*1 and Michael J. Shelley ${ }^{1,2}$ \\ ${ }^{1}$ Applied Mathematics Laboratory, Courant Institute, New York University, USA \\ ${ }^{2}$ Flatiron Institute, Simons Foundation, USA
}

\begin{abstract}
Achieving macroscopic directed migration of microscale swimmers in a fluid is an important step towards utilizing their autonomous motion. It has been experimentally shown that directed motion can be induced, without any external fields, by certain geometrically asymmetric obstacles due to interaction between their boundaries and the swimmers. In this paper, we propose a kinetic-type model to study swimming and directional migration of microscale bimetallic rods in a periodic array of posts with non-circular cross-sections. Both rod position and orientation are taken into account; rod trapping and release on the post boundaries are modeled by empirically characterizing curvature and orientational dependence of the boundary absorption and desorption. Intensity of the directed rod migration, which we call the normalized net flux, is then defined and computed given the geometry of the post array. We numerically study the effect of post spacings on the flux; we also apply shape optimization to find better post shapes that can induce stronger flux. Inspired by preliminary numerical results on two candidate posts, we perform an approximate analysis on a simplified model to show the key geometric features a good post should have. Based on that, three new candidate shapes are proposed which give rise to large fluxes. This approach provides an effective tool and guidance for experimentally designing new devices that induce strong directed migration of microscale swimmers.
\end{abstract}

\section{Introduction}

Microscale swimmers, such as bacteria or chemically active colloids, move autonomously in a fluid by converting energy in the local environment into mechanical work [1, 2]. Possible applications of synthetic microswimmers include drug delivery [3, 4], cargo transport [5], and environmental remediation [6]. One important task in manipulating microswimmers is to achieve their directed macroscopic motion, as opposed to their long-time isotropic motion, which results from a combination of ballistic swimming and angular diffusion [7, 8. Directed migration can be easily induced by externally imposed fields, such as chemical gradient [9, 10, or electromagnetic fields [11, 12, 13, 14. A different approach is to place obstacles in the environment. It has been demonstrated that obstacles can dramatically change the motion of microswimmers. Due to hydrodynamic [15] or steric [16] interactions of swimmers with obstacles, swimmers can aggregate [17, 18, 19, slide [13, 15, 20, hover [21], or even reverse swimming direction [22. There is a developing body of works investigating boundaries of obstacles guiding microswimmers, using flat walls [13, v-shaped funnels 23, 24], spherical obstacles [15, 20, 25], or teardrop-shaped posts 26.

In a series of recent works, microscale bimetallic segmented rods composed of gold and platinum (Au-Pt) have been experimentally studied as a prototype of artificial microswimmers [20, 27. These

*E-mail: jiajun@cims.nyu.edu 


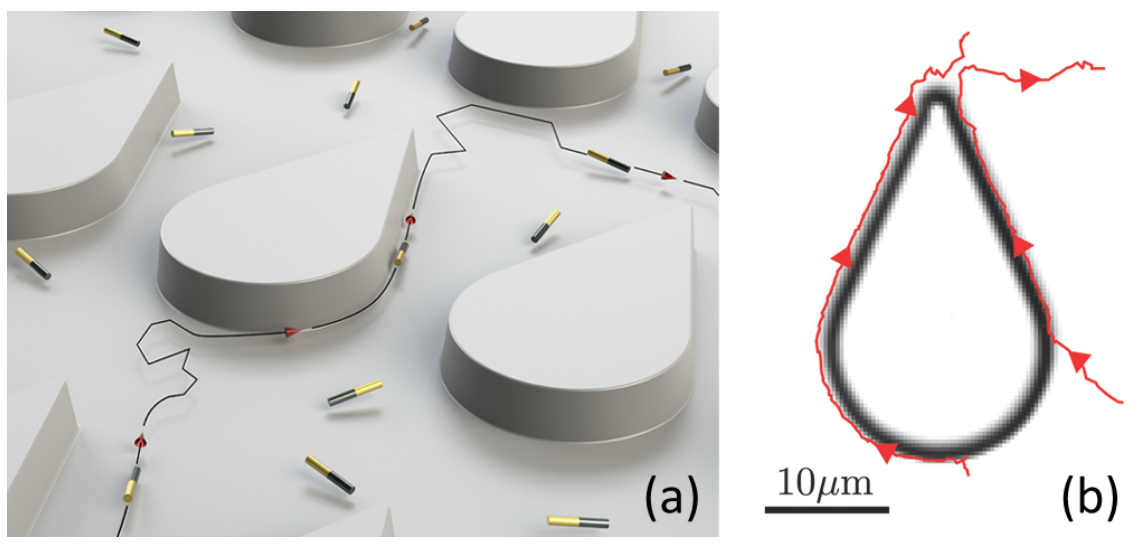

Figure 1: (a) Sketch of bimetallic microscale Au-Pt rods swimming and interacting with an array of teardrop-shaped posts. These rods swim, due to self-electrophoresis, primarily along the bottom or the posts. They move along their axes with the Pt-end leading; their positions and orientations are subject to random fluctuations. After encountering a post, swimming rods tend to travel along the post boundary and preferentially depart from its tip. (b) Experimental observation of two typical trajectories of swimming rods when interacting with a teardrop shaped post. The pictures are adapted from our recent experimental paper [26].

rods, typically $2 \mu \mathrm{m}$ in length and $300 \mathrm{~nm}$ in diameter, move autonomously in aqueous solutions of hydrogen peroxide $\left(\mathrm{H}_{2} \mathrm{O}_{2}\right)$, with the $\mathrm{Pt}$ end leading, due to self-electrophoresis which generates a slip flow along the rod surface [28, 29]. They move with a constant speed along their axes while their positions and orientations are subject to random fluctuations. As they are much denser than water, the Au-Pt rods swim primarily along the microscope coverslip or the obstacles. It is demonstrated in our paper [20] that, these swimming rods can get captured by solid spheres resting on a horizontal plane, and orbit closely around them with little change in their speed, until they are released due to angular diffusion. An uneven spatial distribution of the rods near the spheres and statistics of trapping time are obtained. In a more recent study [26], we show that when the rods swim in a periodic array of teardrop-shaped posts, they interact with the vertical walls of the posts in a similar way; yet the rods preferentially leave the posts at the post's sharp tips due to large boundary curvature there, rendering a statistically biased swimming over long times. See Figure 1 for a sketch of the rods swimming and interacting with an array of teardrop-shaped posts, as well as a picture from the experiment showing typical motion of rods when they encounter a post [26]. It has been experimentally confirmed that the rods are most likely to migrate through the array in the direction pointed by the tips of the teardrop-shaped posts [26].

In this paper, we shall present a kinetic-type model of $\mathrm{Au}-\mathrm{Pt}$ rods swimming in a periodic array of posts with non-circular cross-sections, such as teardrop-shaped posts, and thus generating directed migration over long time. Position and orientation of the rods are both taken into account, as well as the effect of thermal fluctuation. Trapping and release of the rods on the post boundary are modeled via empirically defined rate functions and angular distributions, accounting for curvature and orientational dependence of the boundary absorption and desorption, respectively. Distributions of the rods in the free-space and on the post boundary are found through numerical simulations; the intensity of the directed migration is then defined and calculated.

The degree of directed migration crucially relies on many features of the array, such as the spacings and shapes of the posts in it. We study the effect of post spacing by numerical simulation. We also perform numerical shape optimization to investigate how to choose the shape of posts judiciously so that stronger directed migration of the swimming rods is achieved. General theory of 
shape optimization is well-established from the analysis point of view [30, 31, 32, while numerous applications can be found in many areas of applied mathematics and physics, such as problems involving swimming [33, 34] and fluid motion [35, 36]. In our study, a mathematical derivation of the shape optimization is presented in the Supplementary Materials, together with an iterative optimization method based on an explicitly preconditioned steepest ascent method. We apply shape optimization to two candidate post shapes, and observe a significant increase in directed migration. To better understand the optimization results, we propose a simplified model that well explains the geometric features arising in the optimized shape. This enables us to empirically determine the key geometric ingredients of designing posts. We conclude by giving three new post shapes designed on these ingredients, which does give rise to a significantly stronger directed migration than naive choices.

The rest of the paper is organized as follows. In Section 2.1, we present the model for $\mathrm{Au}-\mathrm{Pt}$ rods swimming in a periodic rectangular array of posts and define the quantity that measures the intensity of the directed migration. The numerical method to compute the ensemble distributions of rod positions and orientations as well as the intensity of the directed migration appear in the Supplementary Materials. In Section 2.2, we formulate the optimization problem seeking better designs of the array so that it induces stronger directed migration. A formal overview of the shape optimization theory, a full derivation of equations involved, and numerical methods for solving these equations and performing shape optimization, are also left to the Supplementary Materials. Dimensionless parameters and rates are specified in Section 3 , while numerical results are presented in Section 4 to study the effects of post spacings and optimization of post shape. To understand key geometric features a good post should have, we perform an approximate analysis to a simplified model in Section 5 . Based on that, three new post designs inducing strong directed migration are proposed in Section 6. We conclude the paper with a brief discussion in Section 7 .

\section{Theory}

\subsection{Modeling microscale swimming rods in a periodic array of posts}

With the typical swimming pattern of the rod described in Section 1 and sketched in Figure 1 . we shall build a kinetic-type model for rods swimming in a periodic array of posts. The model will be presented in a dimensionless manner; the non-dimensionalization will be left to Section 3 . We start from modeling the environment in which the rods are swimming.

Consider a rectangular periodic array of posts printed on the microscope coverslip. The posts are solid cylinders which neither fluid nor the rods can penetrate [26]. A dilute suspension of the $\mathrm{Au}-\mathrm{Pt}$ rods in the aqueous hydrogen peroxide solution is then placed on the coverslip, so that the rods can autonomously swim in the complex landscape. Note that since the rods always swim in a quasi-two-dimensional fashion along the bottom, it suffices to consider the system in two dimensions. Assume the 2-D unit cell of the periodic array of the posts has dimensionless size $a$ and $b$ in $x_{1}$ - and $x_{2}$-directions respectively. We denote the unit cell to be $Y=\left[-\frac{a}{2}, \frac{a}{2}\right] \times\left[-\frac{b}{2}, \frac{b}{2}\right]$. See Figure 2 . The following discussion also applies to unit cells in other shapes with minor modification. For example, for a staggered periodic array, hexagonal unit cells can be more convenient choices than rectangular ones.

In the sequel, for convenience, we will interchangeably use the notion of a post and its crosssection. Let $O$ denote the domain occupied by the post inside $Y$ and let $\omega=Y \backslash O$ be the domain filled with fluid, in which the rods can swim freely. The interior and exterior boundaries of $\omega$ are denoted by $\gamma=\partial O$ and $\partial Y$ respectively; see Figure 2(b).

To study the statistical behavior of the swimming rods, we look at the spatial and orientational distribution of an ensemble of rods in the bulk and on the boundary. We assume that the rod 


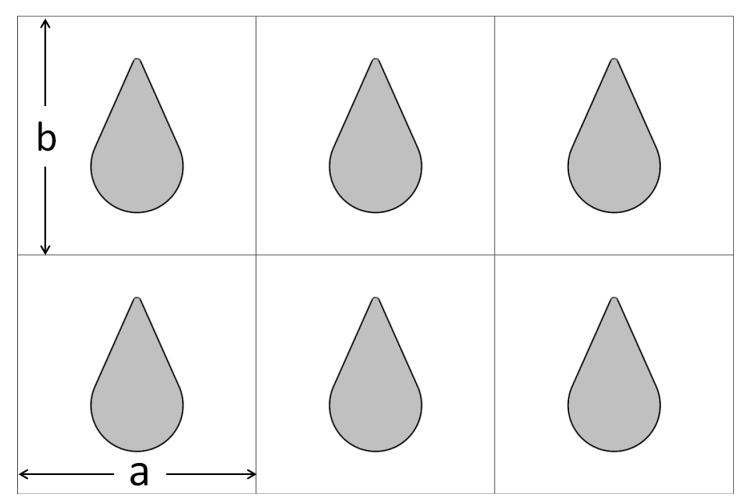

(a)

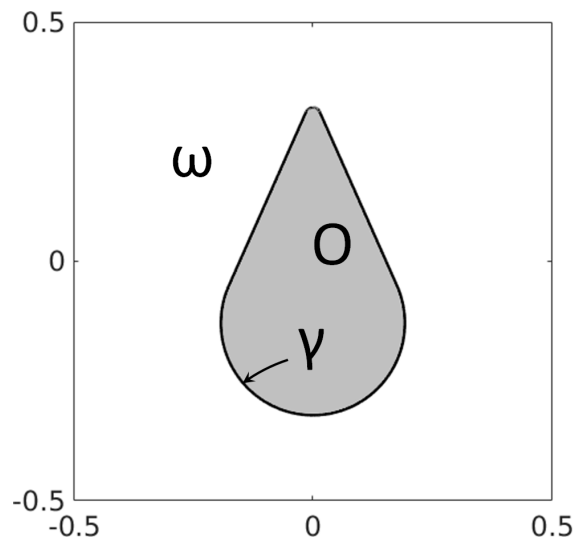

(b)

Figure 2: (a) A periodic array of teardrop-shaped posts. Its unit cells, separated by solid lines, have width $a$ and height $b$; here $a=b=1$. Domains occupied by the posts are marked as grey. (b) An enlarged view of one unit cell in (a). $O$ denotes the grey domain occupied by the post; $\omega$ denotes the exterior domain filled with fluid; $\gamma$ denotes the boundary of $\omega$ between them.

concentration is so small that their interactions are negligible. We also treat the rods as points with orientation but no size.

The motion of the rods is modeled as swimming in the bulk $\omega$, and swimming along the boundary $\gamma$. For a single rod swimming in the bulk, we use $X_{t} \in \omega$ and $2 \pi \Theta_{t}$ to denote its position and orientation, respectively. The latter is the angle between the swimming direction of the rod and the positive $x_{1}$-axis; see Figure 3 . Here $X_{t}$ is defined up to a natural periodicity on the exterior boundary of $\omega$, while $\Theta_{t} \in[0,1)$ defined in the modulus of 1 . We assume that the rod deterministically swim in its axial direction with velocity $v_{0}$, while both its position and orientation are subject to random fluctuations. The stochastic dynamics of $\left(X_{t}, \Theta_{t}\right)$ is then written as follows

$$
\begin{aligned}
& d X_{t}=v_{0}\left(\cos \left(2 \pi \Theta_{t}\right), \sin \left(2 \pi \Theta_{t}\right)\right)^{T}+\sqrt{2 D_{t}} d W_{t}^{(2)}, \\
& d \Theta_{t}=\sqrt{2 D_{r}} d W_{t}^{(1)} .
\end{aligned}
$$

Here $D_{t}$ and $D_{r}$ are scalar (dimensionless) translational and rotational diffusion coefficients, respectively; they are assumed to be constant throughout the state space $\Omega \triangleq \omega \times[0,1] . W_{t}^{(1)}$ and $W_{t}^{(2)}$ are the standard Brownian motions in one and two dimensions, respectively; they are independent with each other. Let $p(x, \theta, t) \geq 0$ be the distribution of rods in $\Omega$, where $x \in \omega$ and $2 \pi \theta \in[0,2 \pi]$. The evolution of $p$ is then governed by the following Fokker-Planck equation associated with 2.1

$$
\partial_{t} p(x, \theta, t)=D_{t} \Delta_{x} p(x, \theta, t)+D_{r} \Delta_{\theta} p(x, \theta, t)-v_{0}(\cos 2 \pi \theta, \sin 2 \pi \theta)^{T} \cdot \nabla_{x} p(x, \theta, t), \quad(x, \theta) \in \Omega .
$$

Here $\Delta_{x}=\partial_{x_{1} x_{1}}+\partial_{x_{2} x_{2}}$, and $\Delta_{\theta}=\partial_{\theta \theta} ; \nabla_{x}=\left(\partial_{x_{1}}, \partial_{x_{2}}\right)^{T}$ is the gradient operator in spatial components only. The term $-v_{0}(\cos 2 \pi \theta, \sin 2 \pi \theta)^{T} \cdot \nabla_{x} p$ represents convection in $\Omega$ due to the directed swimming in the axial direction.

For rods moving along the boundary $\gamma$, we assume they always swim tangentially to $\gamma$; thus only the rod position along $\gamma$ needs to be considered. We also assume that rods swim at the constant speed $v_{0}$ [20] and can never switch swimming direction before it leaves the boundary. This assumption is suitable for bimetallic swimming rods, but might not be true for some biological swimmers [22. For $x \in \gamma$, let $p_{B}^{+}(x, t), p_{B}^{-}(x, t) \geq 0$ be the boundary distributions of rods that swim counterclockwise and clockwise, respectively. The evolution of $p_{B}^{ \pm}$'s is given by

$$
\partial_{t} p_{B}^{ \pm}(x, t)=D_{t} \Delta_{\gamma} p_{B}^{ \pm}(x, t) \mp v_{0} \partial_{\gamma} p_{B}^{ \pm}(x, t)+F_{\text {in }}^{ \pm}(x, t)-F_{\text {out }}^{ \pm}(x, t), \quad x \in \gamma,
$$


where $\Delta_{\gamma}$ and $\partial_{\gamma}$ are the Laplace operator and the derivative along $\gamma$ with respect to its arclength. Here $\gamma$ is parameterized counterclockwise. On the right hand side of (2.3), $D_{t} \Delta_{\gamma} p_{B}^{ \pm}$is the spatial diffusion along $\gamma$; for simplicity, we assume the same diffusion coefficient $D_{t}$ as in the free space. The term $\mp v_{0} \partial_{\gamma} p_{B}^{ \pm}(x, t)$ comes from the deterministic swimming along $\gamma . F_{\mathrm{in}}^{ \pm}(x, t)$ and $F_{\text {out }}^{ \pm}(x, t)$ are rod absorption and desorption fluxes at $x$ on and off the boundary $\gamma$, respectively. They depend on the local geometry of $\gamma$ and how rods hit or leave the boundary.

To model this pair of fluxes, we need some notation. Let $\kappa(x)$ be the curvature of $\gamma$ at $x$, and $\alpha(x)$ be the orientation of the outer normal of $\gamma$ at $x$ with respect to $\omega$, i.e., the normal vector is given by $(\cos \alpha(x), \sin \alpha(x))^{T}$. When a rod appears at a boundary point $x \in \gamma$ with orientational angle $2 \pi \theta$, we define its relative angle with respect to $\gamma$ to be $\beta=2 \pi \theta-\alpha(x)(\bmod 2 \pi)$; see Figure 3 . To this end, we introduce empirical rate functions $r_{\text {in }}(\kappa)$ and $r_{\text {in }}(\kappa)$, and angular functions $\rho_{ \pm}(\beta)$ and $\tau_{ \pm}(\beta)$, to be explained later, and write

$$
\begin{gathered}
F_{\text {in }}^{ \pm}(x, t)=r_{\text {in }}(\kappa(x)) \int_{0}^{1} p(x, \theta, t) \rho_{ \pm}(2 \pi \theta-\alpha(x)) d \theta, \quad x \in \gamma, \\
F_{\text {out }}^{ \pm}(x, t)=r_{\text {out }}(\kappa(x)) p_{B}^{ \pm}(x, t) \int_{0}^{1} 2 \pi \tau_{ \pm}(2 \pi \theta-\alpha(x)) d \theta, \quad x \in \gamma .
\end{gathered}
$$

Here $r_{\text {in }}(\kappa)$ and $r_{\text {out }}(\kappa)$ are called absorption and desorption (Poisson) rates, respectively, which are assumed to be functions of curvature only. In fact, experiments have shown that at least the desorption rate also depends on the rod speed [26]. However, our assumption is valid since the rod speed is fixed to be $v_{0}$. The functions $r_{\text {in }}(\kappa(x))$ and $r_{\text {out }}(\kappa(x))$ then characterize how fast the boundary $\gamma$ can absorb and desorb rods at $x \in \gamma$, respectively. Their precise characterization will be clear after we choose the characteristic scales and do non-dimensionalization in Section 3. The functions $\rho_{ \pm}(\beta)$ account for the orientation dependence in the absorption. We assume that rods hitting $\gamma$ with relative angle $\beta$ will have probabilities $\rho_{+}(\beta)$ and $\rho_{-}(\beta)$ of subsequently swimming counter-clockwise and clockwise along $\gamma$, respectively; see Figure 4(a). To make sense of (2.4), we note that rods hitting $x \in \gamma$ can come from the bulk in all directions; the function $r_{\text {in }}(\kappa(x)) p(x, \theta, t) \rho_{+}(2 \pi \theta-\alpha(x))$ is the amount of rods getting absorbed at $x$ with angle $2 \pi \theta$, and sliding counter-closewise along $\gamma$ afterwards. The function $r_{\text {in }}(\kappa(x)) p(x, \theta, t) \rho_{-}(2 \pi \theta-\alpha(x))$ can be interpreted similarly. If we take an integral over all possible orientations of the incoming rods, we obtain the absorption fluxes.

Similarly, for desorption, rods that are leaving $\gamma$ are assumed to leave at a random angle, with its probability distribution characterized by $\tau_{ \pm}(\beta)$, where $\beta$ is the relative angle defined before. The subscripts \pm indicate the rods originally move counter-clockwise or clockwise before leaving $\gamma$; see Figure 4(b)] We argue as before to obtain 2.5). Via $\beta$ and the tangent direction of $\gamma$, the initial orientational angle of the rod when it returns to the bulk can be determined. This will be useful in deriving the boundary condition of $p$ below.

To summarize, the assumptions on $r_{\text {in }}, r_{\text {out }}, \rho_{ \pm}$and $\tau_{ \pm}$are as follows:

1. $r_{\text {in }}(\kappa), r_{\text {out }}(\kappa) \geq 0$;

2. $\rho_{ \pm}(\beta) \geq 0$ and $\rho_{+}(\beta)+\rho_{-}(\beta) \leq 1$;

3. $\tau_{ \pm}(\beta) \geq 0$ and $\int_{-\pi}^{\pi} \tau_{ \pm}(\beta) \mathrm{d} \beta=1$;

4. $\rho_{+}(\beta)=\rho_{-}(-\beta)$ and $\tau_{+}(\beta)=\tau_{-}(-\beta)$, by symmetry.

Since $\tau_{ \pm}$'s are normalized, Eq. 2.5) reduces to $F_{\text {out }}^{ \pm}=r_{\text {out }} p_{B}^{ \pm}$.

Lastly, the boundary condition of $p$ on $\Gamma \triangleq \gamma \times[0,1]$, the inner curved part of $\partial \Omega$, is derived 


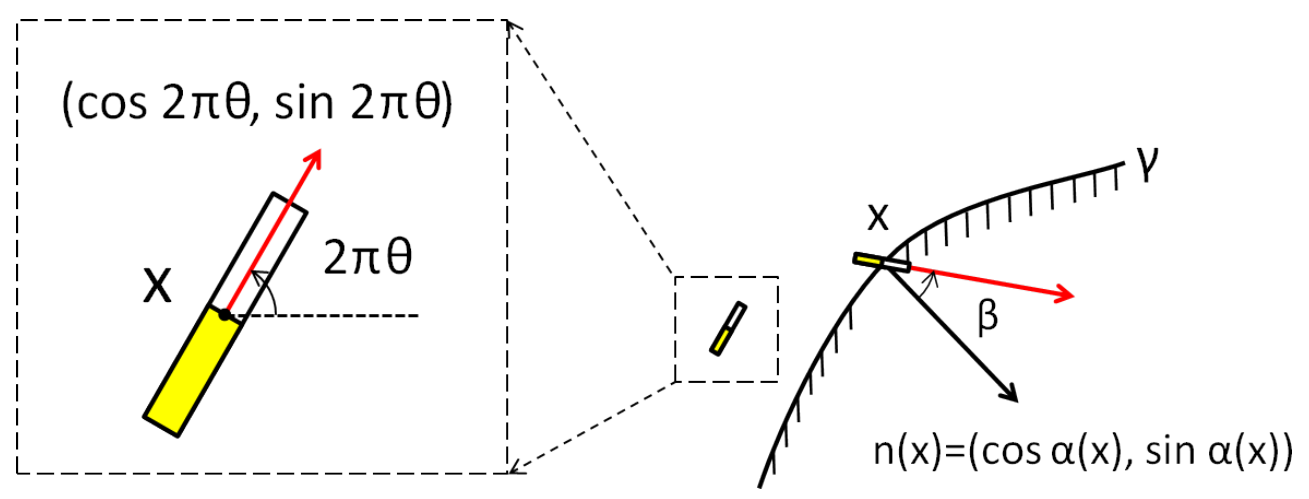

Figure 3: We use $x \in \omega$ and $2 \pi \theta$, with $\theta \in[0,1)$, to represent position and orientational angle of a swimming Au-Pt rod, respectively. The enlarged picture in the big dashed box shows the state $(x, \theta)$ of the rod in the small dashed box, which is swimming away from the post. The red arrow represents its orientation, given by the direction of its $\mathrm{Pt}$ end (white block); the dashed line is the positive $x_{1}$-axis. Note that rods are modeled as points with orientations but no size, although we have drawn a white-yellow rod in the picture for the sake of clarity. In the right half of the figure, with abuse of notations, a rod hitting the post boundary $\gamma$ at $x$ with relative angle $\beta$ is shown. The shaded side of $\gamma$ is occupied by the post. The black arrow is the normal vector of $\gamma$ at $x$, denoted by $n(x)=(\cos \alpha(x), \sin \alpha(x))$, while the red arrow again represents the rod orientation. The (signed) angle between them is the relative angle $\beta$.

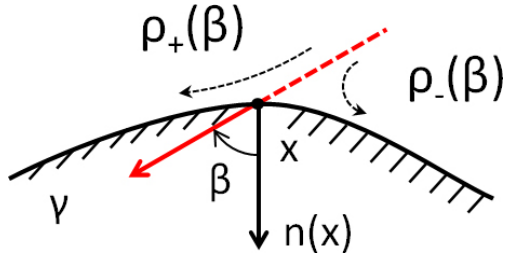

(a)

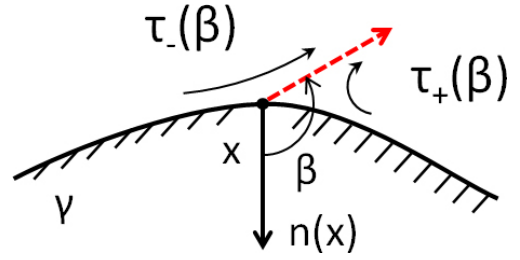

(b)

Figure 4: $\rho_{ \pm}(\beta)$ and $\tau_{ \pm}(\beta)$. The black curve represents $\gamma$ and the shaded side is occupied by the post. The black arrow is the normal vector $n(x)$ of $\gamma$ at $x$, while the red arrow represents the direction in which the rod hits $\gamma$, or the potential direction in which the rod is going to leave $\gamma$. (a) When the rod hits $\gamma$ with relative angle $\beta$, it will have probabilities $\rho_{+}(\beta)$ and $\rho_{-}(\beta)$, respectively, of swimming counter-clockwise and clockwise afterwards along $\gamma$. (b) When a rod is going to leave $\gamma$, its relative angle $\beta$ with respect to $\gamma$ is determined via the distributions $\tau_{ \pm}(\beta)$. The subscripts indicate its original swimming direction before leaving $\gamma$. 
from the conservation law, which gives

$$
\begin{aligned}
D_{t} \frac{\partial p}{\partial n_{\Gamma}}(x, \theta, t)- & v_{0} p(x, \theta, t) \cdot(\cos 2 \pi \theta, \sin 2 \pi \theta, 0) \cdot n_{\Gamma}(x) \\
= & r_{\text {out }}(\kappa(x))\left[p_{B}^{+}(x, t) \cdot 2 \pi \tau_{+}(2 \pi \theta-\alpha(x))+p_{B}^{-}(x, t) \cdot 2 \pi \tau_{-}(2 \pi \theta-\alpha(x))\right] \\
& \quad-r_{\text {in }}(\kappa(x)) p(x, \theta, t)\left[\rho_{+}(2 \pi \theta-\alpha(x))+\rho_{-}(2 \pi \theta-\alpha(x))\right], \quad(x, \theta) \in \Gamma .
\end{aligned}
$$

Here $n_{\Gamma}(x)=(\cos \alpha(x), \sin \alpha(x), 0)^{T}$ is the unit outer normal vector of $\Gamma$ with respect to $\Omega$. The left hand side of 2.6 represents the boundary flux at $(x, \theta) \in \Gamma$ generated by the spatial diffusion and the swimming; it is balanced by the flux coming into the bulk due to desorption and absorption on the right hand side. Using the formula for $n_{\Gamma}, 2.6$ is simplified to be

$$
\begin{aligned}
D_{t} \frac{\partial p}{\partial n_{\Gamma}}(x, \theta, t)-v_{0} p(x, \theta, t) \cos \beta= & 2 \pi r_{\text {out }}(\kappa(x))\left[p_{B}^{+}(x, t) \tau_{+}(\beta)+p_{B}^{-}(x, t) \tau_{-}(\beta)\right] \\
& -r_{\text {in }}(\kappa(x)) p(x, \theta, t)\left[\rho_{+}(\beta)+\rho_{-}(\beta)\right], \quad(x, \theta) \in \Gamma,
\end{aligned}
$$

with $\beta=2 \pi \theta-\alpha(x)$. For the outer flat surfaces of $\partial \Omega$, i.e. $\partial \Omega \backslash \Gamma$, we assign periodic boundary conditions for $p$.

In this paper, we only consider the steady-state solution. Omitting the $t$-dependence in 2.2 and 2.3 , the equations become

$$
\begin{aligned}
& D_{t} \Delta_{x} p(x, \theta)+D_{r} \Delta_{\theta} p(x, \theta)-v_{0}(\cos 2 \pi \theta, \sin 2 \pi \theta)^{T} \cdot \nabla_{x} p(x, \theta)=0 \quad(x, \theta) \in \Omega, \\
& -D_{t} \Delta_{\gamma} p_{B}^{ \pm}(x) \pm v_{0} \partial_{\gamma} p_{B}^{ \pm}(x)=\int_{0}^{1} f_{ \pm}(x, \theta) \mathrm{d} \theta, \quad x \in \gamma, \\
& D_{t} \frac{\partial p}{\partial n_{\Gamma}}(x, \theta)-v_{0} p(x, \theta) \cos \beta+f_{+}(x, \theta)+f_{-}(x, \theta)=0, \quad(x, \theta) \in \Gamma, \\
& p \text { satisfies periodic boundary condition on } \partial \Omega \backslash \Gamma,
\end{aligned}
$$

where $\beta=2 \pi \theta-\alpha(x)$ and

$$
f_{ \pm}(x, \theta)=r_{\mathrm{in}}(x) \rho_{ \pm}(\beta) p(x, \theta)-r_{\text {out }}(x) \cdot 2 \pi \tau_{ \pm}(\beta) p_{B}^{ \pm}(x), \quad(x, \theta) \in \Gamma .
$$

As we are describing the probability distribution of rods, the following normalization condition is needed

$$
N(\Omega) \triangleq \int_{\Omega} p(x, \theta) \mathrm{d} x \mathrm{~d} \theta+\int_{\gamma}\left[p_{B}^{+}(x)+p_{B}^{-}(x)\right] \mathrm{d} \gamma=1 .
$$

Note that if 2.12) is not assumed, that $\left(p, p_{B}^{+}, p_{B}^{-}\right)$is a solution of the equations 2.7)-2.11 implies that $\left(\lambda p, \lambda p_{B}^{+}, \lambda p_{B}^{-}\right)$is also a solution for $\forall \lambda>0$.

Suppose we have obtained a nontrivial solution $\left(p, p_{B}^{+}, p_{B}^{-}\right)$to 2.7)-2.11 (obviously $(0,0,0)$ is a trivial solution which is not interesting), without necessarily satisfying 2.12. We wish to characterize the intensity of the spontaneous directed migration of the rods induced by the post in some particular direction, say the positive $x_{2}$-direction. In our model, it is exactly the probability flux crossing the part of $\partial \Omega$ where $x_{2}=b / 2$. The unnormalized net flux is defined to be

$$
F(\Omega)=\int_{\partial \Omega \cap\left\{x_{2}=b / 2\right\}}-D_{t} \frac{\partial p}{\partial x_{2}}+v_{0} p \sin 2 \pi \theta d A .
$$

The first term comes from the spatial diffusion of rods, while the second is due to the directed swimming, where the rod orientation plays a role. Since this flux is generated by an amount of rods given by $N(\Omega)$, the normalized net flux is thus defined to be $E(\Omega) \triangleq F(\Omega) / N(\Omega)$. $E(\Omega)$ will be the key quantity in the rest of the paper.

The numerical method for solving the coupled system 2.7 - 2.11) and computing $E(\Omega)$ will be given in the Supplementary Materials. 


\subsection{Seeking stronger directed migration of the rods}

It is clear that the intensity of the directed migration of the rods is governed by many geometric features of the array, such as the sizes of gaps between neighboring posts, and the shapes of posts. An interesting and practical question to ask is how to make the directed migration stronger by cleverly designing the array and post shapes. In our model, this could be formulated as the following optimization problem: given the functions $r_{\text {in }}(\kappa), r_{\text {out }}(\kappa), \rho_{ \pm}(\beta)$ and $\tau_{ \pm}(\beta)$, find $Y=\left[-\frac{a}{2}, \frac{a}{2}\right] \times$ $\left[-\frac{b}{2}, \frac{b}{2}\right]$ and $\omega \subset Y$, such that $E(\Omega)$ is maximized.

This is an infinite dimensional optimization problem and it would be very hard to find the actual maximizer. We are only able to pose it formally. Our strategy here is as follows. Firstly, we shall study how the spacings of the posts affect $E(\Omega)$, simply by fixing the shape of the post and tuning $a$ and $b$ to see how $E(\Omega)$ changes. Secondly, we would like to look for a better shape of the posts. Using the theory of shape optimization, we develop a numerical method that evolves $\gamma$ in an iterative manner, so that the corresponding $E(\Omega)$ keeps increasing with each iteration. The mathematical formulation of the shape optimization problem is exceedingly long. We shall leave an overview of the theory of shape optimization, a complete derivation of the equations needed in the shape optimization, and an introduction of the associated numerical method to the Supplementary Materials.

\section{Model Choices}

Before presenting numerical results, we specify the dimensionless parameters and rates used in the simulations.

We take the characteristic length scale in the model to be the typical size of the unit cell in the experiment 26], which is $L=45 \mu \mathrm{m}$. We use the angular diffusion of the rods to determine the characteristic time scale. In the experiments, the typical angular diffusion scale is $0.5 \mathrm{rad} / \mathrm{s}$. Hence, we take the characteristic time scale to be $T=4 \pi s$. In this way, the dimensionless angular diffusion coefficient is $D_{r}=1$. Note that it is $2 \pi \theta$ instead of $\theta$ that represents the rod orientation.

Under the above choice, we take the dimensionless parameter in the model as follows: $D_{t}=0.002$, $D_{r}=1$, and $v_{0}=1$. Indeed, this corresponds to the case where the spatial diffusion of rods is approximately $0.002 \cdot[45 \mu \mathrm{m}]^{2} /[4 \pi \mathrm{s}] \approx 0.322 \mu \mathrm{m}^{2} / \mathrm{s}$; the angular diffusion is $0.5 \mathrm{rad} / \mathrm{s}$; and rods swim with approximate speed $1 \cdot[45 \mu \mathrm{m}] /[4 \pi \mathrm{s}] \approx 3.58 \mu \mathrm{m} / \mathrm{s}$. All of these agree qualitatively with the measurements in the typical experiments [26].

There has been little systematic measurement of $r_{\text {in }}(\kappa), r_{\text {out }}(\kappa), \rho_{ \pm}(\beta)$ and $\tau_{ \pm}(\beta)$. However, some experiments indicate that for swimming Au-Pt rods [26] or swimming Janus particles [25], $r_{\text {out }}(\kappa)$ is an increasing function of the dimensionless curvature $\kappa$. In our study of the hydrodynamic capture of bacteria to a solid surface, a threshold $\pi / 9$ of the take-off angle is used in the stochastic simulation of a lubrication theory model to fit an exponential distribution to the trapping times [20]. With this in mind, we use functions above such that they qualitatively agree with the existing experimental observations and physical intuition. We take $r_{\text {in }}(\kappa)=1$, which implies that each segment of the post boundary with equal length has equal efficacy in absorbing rods, regardless of its curvature. More precisely, given the unit density of rods (one rod per unit cell on average, i.e. $\frac{1}{45^{2}} \operatorname{rod} / \mu m^{2}$ ) in the vicinity of the post boundary, there is on average one absorbing event occurring over a boundary section with arclength $45 \mu m$ every $4 \pi s \approx 12.6 s$, which is on the right scale. The function $r_{\text {out }}(\kappa)$ is defined as the inverse of the expected trapping time of rods sliding along a circular boundary with constant dimensionless curvature $\kappa$. We take

$$
r_{\text {out }}(\kappa)=\frac{10}{\pi} \arctan \left(\frac{\kappa-20}{4}\right)+5
$$

as a convenient choice; see Figure 5(a). Indeed, we only need the property that $r_{\text {out }}(\kappa)$ is increasing 

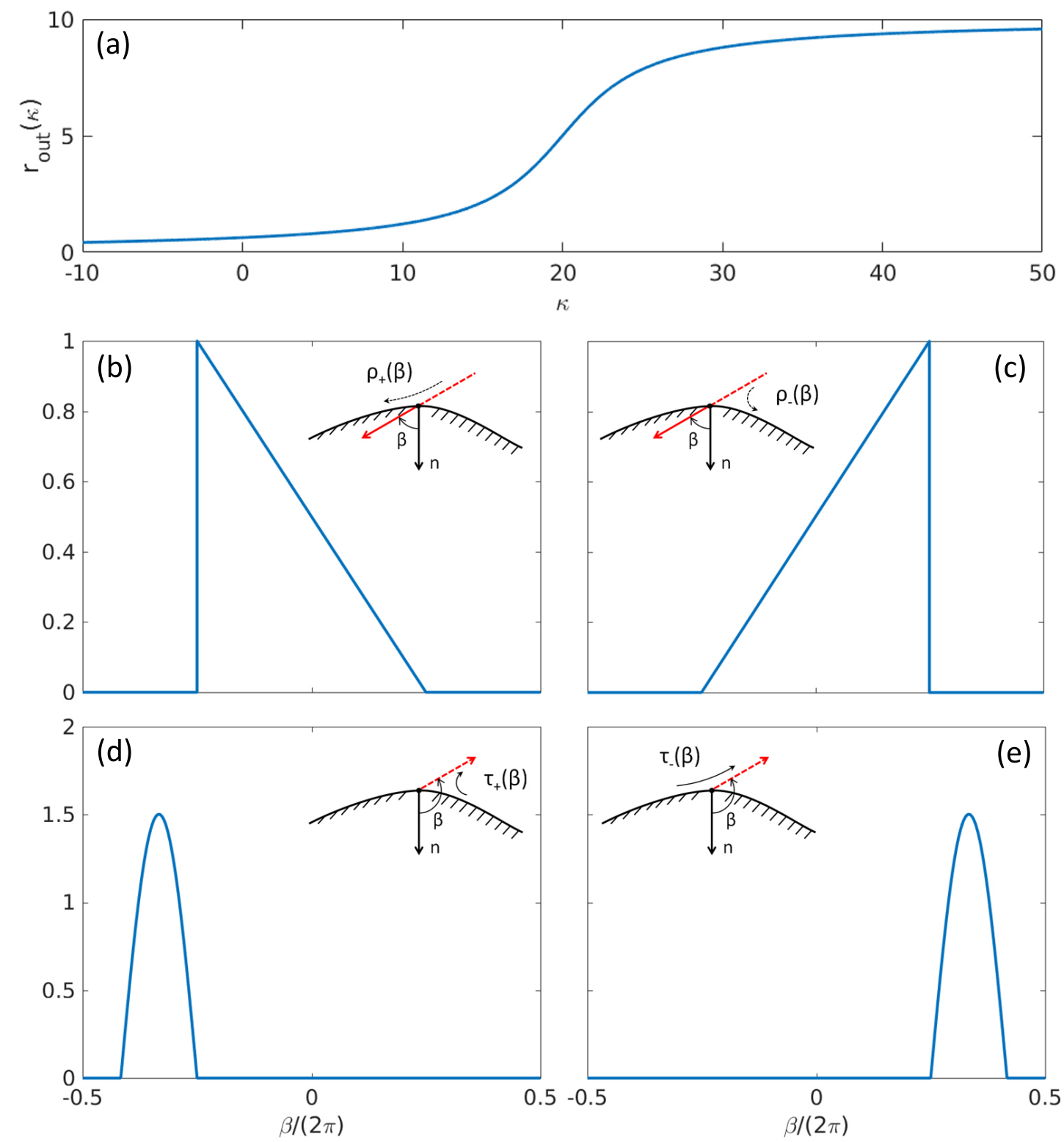

Figure 5: Functions $r_{\text {out }}(\kappa), \rho_{ \pm}(\beta)$ and $\tau_{ \pm}(\beta)$ used in the simulations. (a) The graph of $r_{\text {out }}(\kappa)=$ $\frac{10}{\pi} \arctan \left(\frac{\kappa-20}{4}\right)+5$. It is positive, increasing in $\kappa$ and bounded as $\kappa \rightarrow+\infty$. (b-e) Graphs of functions $\rho_{ \pm}(\beta)$ and $\tau_{ \pm}(\beta)$ used in the simulations. The insets illustrate the situations where these functions come into play; see $(3.2),(3.3)$ and Figure 4 for more details. Note that $\tau_{ \pm}$are normalized in the sense that $\int_{-\pi}^{\pi} \tau_{ \pm}(\beta) d \beta=1$. 
in $\kappa$, which implies that the efficacy of the boundary in releasing rods is higher where its curvature is larger (more convex). That $r_{\text {out }}(\kappa)$ saturates as $\kappa \rightarrow+\infty$ is a convenient feature that makes the numerical simulations more tractable. We shall discuss its effect in Section 4.2. To give a sense of the choice of $r_{\text {out }}(\kappa)$, we have $r_{\text {out }}(20)=5$, which implies that when a rod is sliding along the boundary of a circular post with radius $2.25 \mu \mathrm{m}=[45 \mu \mathrm{m}] / 20$, the expected sliding time is $\frac{4 \pi}{5} s \approx 2.51 \mathrm{~s}$. In other words, the Poisson leaving rate of a rod on a circular boundary with radius $2.25 \mu \mathrm{m}$ is approximately $0.398 \mathrm{~s}^{-1}$, which agrees qualitatively with the experimental data [26].

The functions $\rho_{ \pm}(\beta)$ are taken to be

$$
\rho_{ \pm}(\beta)=\mp \frac{1}{\pi} \beta+\frac{1}{2}, \quad \beta \in[-\pi / 2, \pi / 2],
$$

and $\rho_{ \pm}(\beta) \equiv 0$ for $\beta \in[-\pi, \pi / 2) \cup(\pi / 2, \pi)$. They are plotted in Figure 5(b) and 5(c). They imply that when a rod hits the boundary perpendicularly $(\beta=0)$, it has equal probability of going in either direction along the boundary. When it approaches in the tangent directions $(\beta= \pm \pi / 2)$, it will go forward in that direction with probability 1 . When a rod reaches the boundary with its orientation pointing away from the boundary $(\beta \in[-\pi, \pi / 2) \cup(\pi / 2, \pi))$, it will not get absorbed. Indeed, the last case can arise when spatial diffusion pushes the rod to the boundary even though its orientation points away.

The functions $\tau_{ \pm}(\beta)$ are defined as follows. Let

$$
\tau_{+}(\beta)= \begin{cases}\frac{3}{2} \cos (3 \beta) & \text { for } \beta \in[-5 \pi / 6,-\pi / 2], \\ 0 & \text { for } \beta \in[-\pi,-5 \pi / 6) \cup(-\pi / 2, \pi) .\end{cases}
$$

We then define $\tau_{-}(\beta)=\tau_{+}(-\beta)$. These are plotted in Figure 5(d) and 5(e). In this setting, rods are going to leave the boundary with take-off angles, defined to be the (unsigned) angle between the boundary and the rods, ranging from 0 to $\pi / 3(\beta \in[-5 \pi / 6,-\pi / 2]$ for rods originally sliding counter-clockwise on $\gamma$, or $\beta \in[\pi / 2,5 \pi / 6]$ for rods originally sliding clockwise). The most likely take-off angle is $\pi / 6$ ( $\beta=-2 \pi / 3$ for rods originally sliding counter-clockwise, or $\beta=2 \pi / 3$ for rods originally sliding clockwise).

\section{Numerical Results}

With implementation details now behind us, in the section, we shall present numerical results concerning how post spacings and post shapes can affect the induced normalized net flux $E(\Omega)$.

\subsection{The effect of post spacings}

Our first numerical simulation is devoted to investigating how post spacings affect the normalized net flux $E(\Omega)$. To be more precise, we fix the shape of the post specified later, and change size of the unit cell specified by $a$ and $b$, to see how $E(\Omega)$ changes correspondingly. Note that the set $\Omega$ implicitly depends on both $a$ and $b$.

The post used in this simulation has a teardrop-shaped cross-section, defined by two circular arcs smoothly connected by two straight lines; see Figure 2(b). Under the non-dimensionalization, the radii of the larger and the smaller circular arcs are 0.192 and 0.0154 respectively. Their corresponding dimensionless curvatures $\kappa$ are 5.21 at the large end and 65.1 at the small tip, respectively. The angle between the two straight sides is 48 degrees. The tip of the post points to the positive $x_{2}$-direction. The dimensionless size of the post is approximately 0.642 in the vertical direction and 0.384 in the horizontal direction. Its perimeter is approximately 1.59 .

In Figure 6, we show how $E(\Omega)$ changes as we vary the dimensionless width $a$ and height $b$ of the unit cell. These results are obtained by solving the model 2.7 -2.11 for each pair of $(a, b)$ 


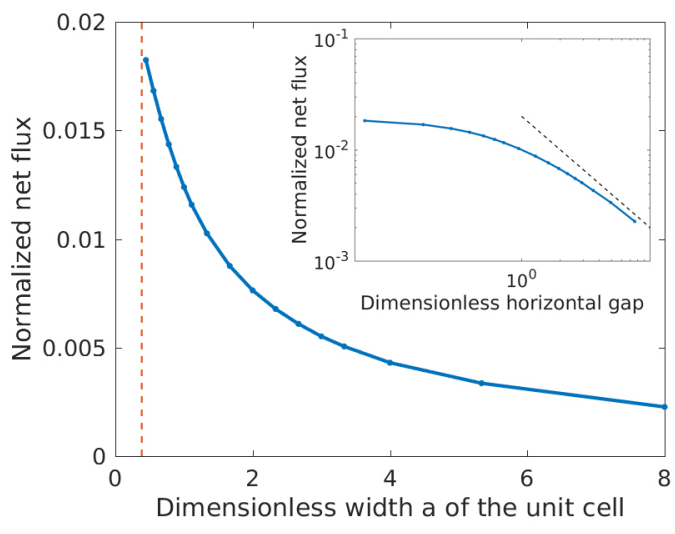

(a)

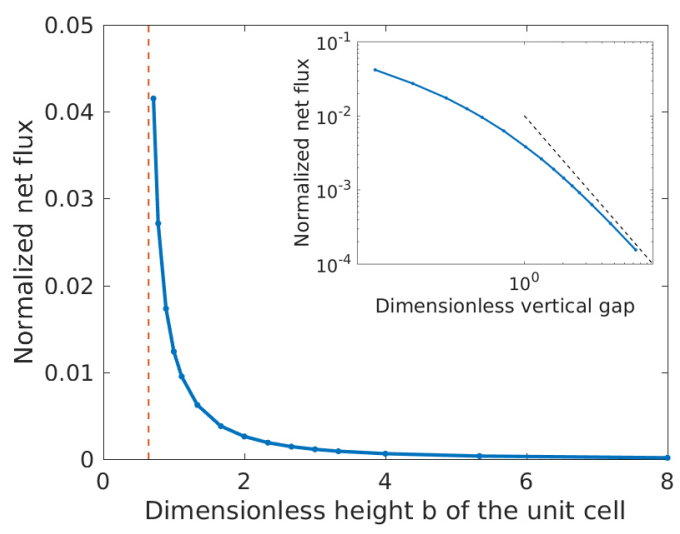

(b)

Figure 6: The normalized net flux $E(\Omega)$ depending on the dimensionless width $a$ and the height $b$ of the unit cell. (a) With $b=1, E(\Omega)$ decreases as $a$ increases. The red dashed line indicates the minimum possible width $a_{\text {min }}=0.384$. The inset shows a log-log plot of $E(\Omega)$ depending on the dimensionless horizontal gap, $a-a_{\min }$. The black dashed line in the inset has slope -1 . (b) With $a=1, E(\Omega)$ decreases as $b$ increases. The red dashed line indicates the minimum possible height $b_{\min }=0.642$. The inset is a log-log plot of $E(\Omega)$ depending on the dimensionless vertical gap, $b-b_{\min }$. The black dashed line in the inset has slope -2 .

and calculating the corresponding $E(\Omega)$. In Figure 6(a) we fix $b=1$ and vary $a$. It shows that $E(\Omega)$ decreases as $a$ increases. The red dashed line indicates the minimum $a\left(a_{\min }=0.384\right)$ we can possibly achieve, in which case the neighboring teardrop-shaped posts will touch each other. As $a$ approaches $a_{\min }, E(\Omega)$ converges a finite value. The inset of Figure $6(\mathrm{a})$ shows a log-log plot of $E(\Omega)$ vs. $a-a_{\min }$. The black dashed line in the inset has slope -1 , which implies that when $a$ is large, $E(\Omega)$ decays like $\left(a-a_{\text {min }}\right)^{-1}$, or equivalently, as $a^{-1}$. In Figure 6(b), we take $a=1$ and vary $b$. $E(\Omega)$ decreases as $b$ increases. The red dashed line again indicates the minimum $b\left(b_{\min }=0.642\right)$ we can achieve, when the teardrop-shaped posts in two neighboring rows will touch each other. When $b$ approaches $b_{\min }, E(\Omega)$ apparently diverges to infinity. The inset of Figure 6(b) shows a log-log plot of $E(\Omega)$ vs. $b-b_{\min }$. The black dashed line in the inset has slope -2 . In other words, when $b$ is large, $E(\Omega)$ decays like $\left(b-b_{\min }\right)^{-2}$, or equivalently $b^{-2}$.

The above results agree with the intuition that the open-space swimming away from the boundary smears out the anisotropy or bias [26] in the swimming direction induced by the boundary shape, thus making $E(\Omega)$ weaker. As a result, $E(\Omega)$ should get boosted if we compress the open space by shrinking the gaps between neighboring posts in both directions. Indeed, shrinking $b$ might be particularly effective due to the unboundedness of the graph in Figure $6(\mathrm{~b})$ when $b \rightarrow b_{\min }$. However, we should remark that making the gaps too narrow is not always favored in practice. Swimming rods can hit into each other or even cause traffic jams in very narrow gaps, which impairs their mobility and makes the directed migration weaker. Narrow gaps may significantly change the swimming behavior of the self-propelled rods, which is not considered in our model. For example, experiments show that active rods can increase speed by up to five times in confining channels with ceiling [37]. Further, the size of the rods, which is ignored in our model, becomes important when they swim in confined spaces like narrow gaps.

With this in mind, we study the net flux under a different normalization by normalizing the number of rods per unit area instead of one unit cell. This is more useful in practice, since we may use suspensions of swimming rods with some particular concentration to do experiments, and so the 
number of rods in the unit area is approximately given. Besides, we may want to compute the net flux per unit width of array, since that characterizes the efficacy of the whole device in transporting rods within horizontal cross sections of unit length. The net flux under this new normalization is thus given by $\tilde{E}(\Omega)=a b E(\Omega) / a=b E(\Omega)$, which is the net flux over unit horizontal cross section with the rod concentration is normalized.

From the discussion above, it is known that when $a$ or $b$ is large, $\tilde{E}(\Omega)$ should decay like $a^{-1}$ or $b^{-1}$ respectively. This can be justified by the following formal analysis.

1. When $b$ is fixed and $a$ is large, posts in different vertical columns function almost independently as they are so far away. That $a$ gets doubled is almost equivalent to removing half of the posts in one row, which will naturally decrease $\tilde{E}(\Omega)$ by one half. This implies that $\tilde{E}(\Omega)$ will decay like $a^{-1}$ when $a$ is large.

2. When $a$ is fixed and $b$ is large, posts in different rows are now far away from each other. If a rod leaves a post and swims into the open space towards posts in another neighboring row, then midway its orientation is already randomized. An important length scale here is $v_{0} / D_{r}=1$, which is the characteristic distance a rod can travel away from the post before it forgets its initial direction when leaving the boundary. Therefore, $p(x, \theta)$ should have little $\theta$-dependence when the distance between $x$ and any posts in the array is significantly larger than 1 . As a result, when $b$ is large, the motion of the rods midway between two rows can be characterized by an enhanced isotropic diffusion with the effective diffusion coefficient $D_{\text {eff }}=D_{t}+\frac{v_{0}^{2}}{4 D_{r}}$ 7. Instead of (2.7), one can solve $D_{\text {eff }} \Delta \tilde{p}(x)=0$ for the spatial distribution of rods, while the orientational distribution of rods there should be almost uniform in all directions. From the far-field point of view, the effect of one post can be modeled as a dipole in the positive $x_{2}$-direction, since it effectively sucks rods from one end (the larger end) and releases them from the other (the smaller tip). The magnitude of the dipole is insensitive to $b$, since we have normalized the rod concentration instead of the number of rods per unit cell. Hence, the far-field spatial density of rods $\tilde{p}(x)$ induced by one single dipole at the origin, up to an additive constant, should be approximately of the form

$$
\tilde{p}(x) \sim \frac{C x_{2}}{x_{1}^{2}+x_{2}^{2}},
$$

where $C$ is a constant depending on $D_{\text {eff }}$ and the dipole magnitude.

We wish to align such dipole in an array with horizontal spacing $a$ and vertical spacing $b$, and calculate the net flux crossing the segment $\left\{\left(x_{1}, x_{2}\right): x_{1} \in[-a / 2, a / 2], x_{2}=b / 2\right\}$. We start from one row of posts all centered at $x_{2}=0$. The spatial density in a neighborhood of the line $x_{2}=b / 2$ contributed by this row should be well approximated, up to an additive constant, by $\tilde{p}_{0}(x)=\sum_{k \in \mathbb{Z}} \tilde{p}\left(x_{1}+k a, x_{2}\right)$. According to 2.13), we calculate the net flux contributed by this row as

$$
\tilde{E}_{0}=\int_{\partial \Omega \cap\left\{x_{2}=b / 2\right\}}-D_{t} \frac{\partial \tilde{p}_{0}}{\partial x_{2}}+v_{0} \tilde{p}_{0} \sin 2 \pi \theta d A .
$$

The subscripts 0 implies this portion of the net flux comes from the row of posts centered at $x_{2}=0$ (i.e. $\tilde{p}_{0}$ ). The second term in the integral above should vanish, since there is no 
$\theta$-dependence in $\tilde{p}_{0}(x)$ and the $\theta$-integral of $\sin 2 \pi \theta$ is zero. Hence,

$$
\begin{aligned}
\tilde{E}_{0} & =-D_{t}\left[\int_{-a / 2}^{a / 2} \frac{\partial \tilde{p}_{0}}{\partial x_{2}} d x_{1}\right]_{x_{2}=b / 2}=-D_{t} \sum_{k \in \mathbb{Z}}\left[\int_{-a / 2}^{a / 2} \frac{\partial \tilde{p}\left(x_{1}+k a, x_{2}\right)}{\partial x_{2}} d x_{1}\right]_{x_{2}=b / 2} \\
& =-D_{t} \sum_{k \in \mathbb{Z}}\left[\int_{k a-a / 2}^{k a+a / 2} \frac{\partial \tilde{p}\left(x_{1}, x_{2}\right)}{\partial x_{2}} d x_{1}\right]_{x_{2}=b / 2}=-D_{t}\left[\int_{\mathbb{R}} \frac{\partial \tilde{p}}{\partial x_{2}} d x_{1}\right]_{x_{2}=b / 2} \\
& =-D_{t}\left[\int_{\mathbb{R}} \partial_{x_{2}}\left(\frac{C x_{2}}{x_{1}^{2}+x_{2}^{2}}\right) d x_{1}\right]_{x_{2}=b / 2}=\frac{C_{0}}{b},
\end{aligned}
$$

where $C_{0}$ is a positive constant depending on $D_{t}, D_{\text {eff }}$ and the dipole magnitude. By symmetry, the net flux contributed by the row of posts centered at $x_{2}=b$ should be $\tilde{E}_{1}=\tilde{E}_{0}$. For the rows of posts farther away from the line $x_{2}=b / 2$, their contributions to the net flux are suppressed by the screening effect of the rows that are closer to $x_{2}=b / 2$. Indeed, the probability of a rod leaving from a post centered at $x_{2}=k b$ and reaching the horizontal line $x_{2}=b / 2$ without being captured any other posts one the way should decay exponentially as $|k-1 / 2| \rightarrow \infty$; in other words, the contributions to the net flux from these farther rows cannot be fully seen by the line $x_{2}=b / 2$ due to the existence of closer rows. Assume the screening factor to be $\alpha_{k}$ for the row of posts centered at $x_{2}=k b$, with $\sum_{k \in \mathbb{Z}} \alpha_{k}<\infty$. We write the contribution of the row centered at $x_{2}=k b$ to the net flux crossing the segment $\left\{\left(x_{1}, x_{2}\right): x_{1} \in[-a / 2, a / 2], x_{2}=b / 2\right\}$ to be $\tilde{E}_{k}=\alpha_{k} \tilde{E}_{0}$. Presumably, $\alpha_{k}$ 's should be independent of $b$, but only depend on the horizontal spacing of neighboring posts in one row, which is fixed here. The total net flux induced by the whole array then becomes

$$
\tilde{E}(\Omega)=\sum_{k \in \mathbb{Z}} \alpha_{k} \tilde{E}_{0}=\frac{\tilde{C}}{b},
$$

where $\tilde{C}$ is a constant depending on $\alpha_{k}$ 's, $D_{t}, D_{\text {eff }}$ and the dipole magnitude, but independent of $b$. This justifies the $b^{-1}$-decay of $\tilde{E}(\Omega)$ when $b$ is large.

\subsection{Optimization of post shape}

In the second family of simulations, we fix the size of the unit cell by setting $a=b=1$, and apply the shape optimization method mentioned in Section 2.2 to find posts that induce larger normalized net flux $E(\Omega)$. See Section $\mathrm{C} / \mathrm{E}$ for the complete mathematical derivations of the equations involved, and see Section $\mathrm{F}$ for the numerical methods. The parameters and rates have been chosen in Section 3.

We start from the post in the convex teardrop shape introduced in Section 4.1. Figure 7(a) shows the comparison before and after we apply the shape optimization to the teardrop-shaped candidate. The blue curve represents the initial shape while the red curve is the optimized one (the final shape). Note that the iterative optimization gets terminated when the post gets too close (dimensionless distance $\leq 0.02)$ to the border of the unit cell. We observe that with shape optimization, the post swells significantly and becomes non-convex. A small round head forms at the top, connected by a thin neck with the larger belly. The dimensionless curvature around the head is in a narrow range $[24.5,25.5]$; recall that it is 65.1 at the tip of the initial shape. The side parts of the post, initially flat, become slightly wavy. This can be artificial since only low-frequency modes are used in describing the boundary evolution. Indeed, we use 240 equally-spaced points to represent the curve, but only the first 41 Fourier modes are used in the boundary evolution. See Section $\mathrm{F}$ for more details about the numerical method. The lower half of the post largely remains a circular arc. In the course of the shape optimization, $E(\Omega)$ increases almost seven-fold from $1.24 \times 10^{-2}$ to $8.44 \times 10^{-2}$.

To investigate how rods are spatially distributed in the presence of the posts with the initial or the optimized shapes in Figure 7(a), we plot in Figure 7(b) and 7(c) their corresponding spatial 


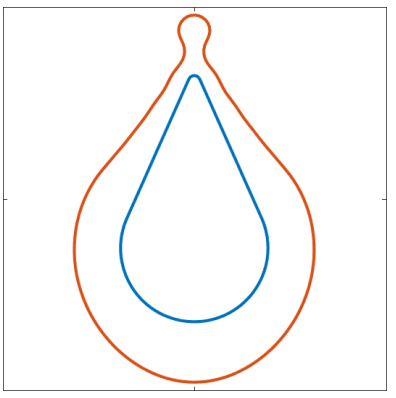

(a)

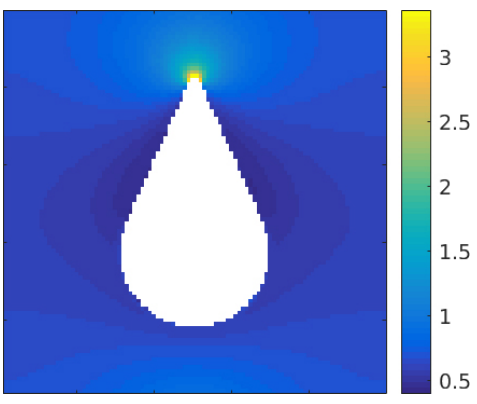

(b)

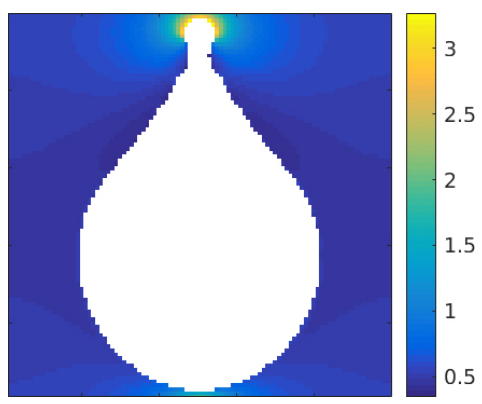

(c)

Figure 7: Performing shape optimization of a teardrop-shaped post. (a) The initial and the optimized shapes are represented by blue and red curves, respectively. In the course of the shape optimization, the post swells, with the sharp tip at the top evolving into a small round head. The lower half of the post remains a circular arc, while the overall post shape becomes non-convex. $E(\Omega)$ increases from $1.24 \times 10^{-2}$ to $8.44 \times 10^{-2}$. (b-c) The spatial concentrations of rods $c(x)$ in the presence of the posts with the initial and the optimized shapes in (a) are plotted in (b) and (c), respectively. The fraction of rods captured on the post boundary is not included in these figures.

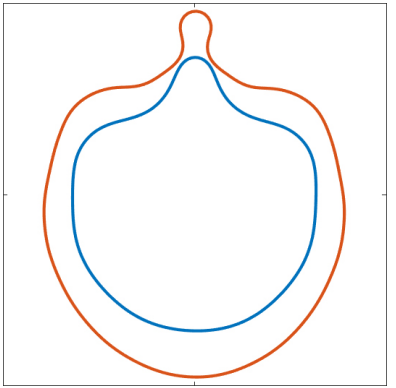

(a)

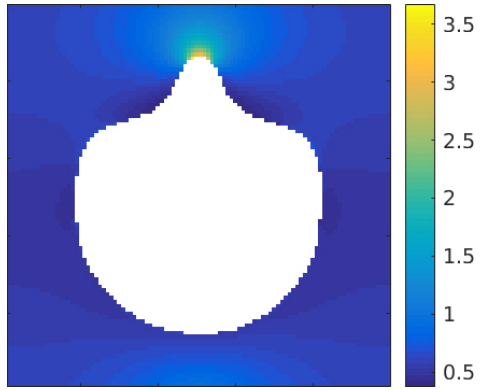

(b)

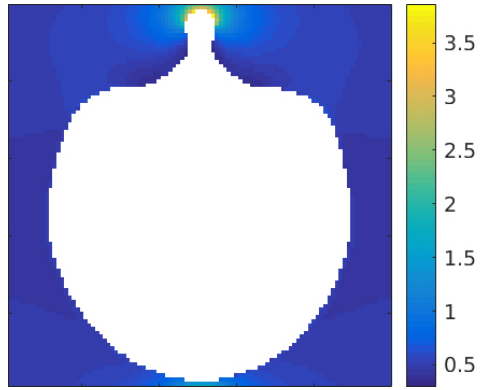

(c)

Figure 8: Performing shape optimization of a nut-shaped post. (a) The initial and the optimized shapes are represented by blue and red curves, respectively. In the course of the shape optimization, the post swells and a small round head forms at the top, while the lower half of the post remains a circular arc. $E(\Omega)$ increases from $1.81 \times 10^{-2}$ to $7.82 \times 10^{-2}$. (b-c) The spatial concentrations of rods $c(x)$ in the presence of the posts with the initial and the optimized shapes in (a) are plotted in (b) and (c), respectively. Again, the fraction of rods captured on the post boundary is not included. 
concentrations of rods $c(x) \triangleq \int_{0}^{1} p(x, \theta) d \theta$ for $x \in \omega$. Note that the fraction of rods captured on the boundary is not included in $c(x)$. Figure $7(\mathrm{~b})$ and $7(\mathrm{c})$ share some common features:

1. The rod concentrations in both cases have their peaks near the top of the posts, implying that the top parts are the sites where strong net desorption occurs.

2. There are regions of depletion near the flat sides of the posts in both cases.

3. The rod concentrations around the bottoms are also relatively high, due to the high concentrations near the tops of the posts in the next unit cells right below.

4. The rod concentrations in both cases have a negative normal derivative near the bottom of the posts (i.e. $c(x)$ decreases when we approach the bottom from some distance away), which implies that the bottoms are effectively absorbing rods.

The main difference between Figure $7(\mathrm{~b})$ and $7(\mathrm{c})$ is that, the area of the strong desorption site at the top in Figure $7(\mathrm{c})$ is much larger than that in Figure $7(\mathrm{~b})$, although the curvature there $(\kappa \in[24.5,25.5])$ is much smaller than that $(\kappa=65.1)$ in the initial shape. This can partially explain why the optimized shape can induce a much larger net flux.

Next, we apply the shape optimization to a new nut-shaped post. The motivation of choosing this as the new initial shape is that the head-forming process can potentially increase $E(\Omega)$. It might accelerate this process if we start from an initial shape which already has a head. In Figure $8(\mathrm{a})$, we plot the shape of post before and after the shape optimization. Again, the blue curve represents the initial shape while the red curve is the final one. The normalized net flux increases from $1.81 \times 10^{-2}$ to $7.82 \times 10^{-2}$, which is a big improvement but not as good as the previous case. We see that once again, the shape swells to fill almost the whole height of the unit cell; a round head and a neck forms at the top, while the lower half of the post largely remains a circular arc. The dimensionless curvature at the head of the final shape ranges in $[24,25.5]$. Again we plot the spatial concentrations of rods corresponding to posts with the initial and optimized shapes in Figure 8(b) and 8(c) respectively. It is clear that the top parts of both posts are the strong desorption sites, while it is larger in the optimized shape than in the initial shape. Besides, there are noticeably two more sites near the post boundary with relatively high rod concentration and positive normal derivatives of $c(x)$. They are the "shoulders" of both the initial and the optimized shapes, which refer to the curved parts on both sides of the posts; they are also efficient in releasing rods. The bottoms of the posts are the main absorption sites as before.

The effect of the "shoulders" on the net flux is unclear though. The net flux can benefit from larger desorbing sites. On the other hand, however, the "shoulders" are not as efficient desorbing sites as the heads at the top, since the "shoulders" are far away from the top border of the unit cell and the rods released there may fail to reach the top border. In this sense, the presence of the "shoulders" can impair the capability of the head releasing rods at the top, and thus reduce $E(\Omega)$. This may explain why the optimized shape in this case does not have as high $E(\Omega)$ as the one in the Figure $7(\mathrm{a})$.

It is shown in Section 4.1 that shrinking gaps between neighboring posts, especially the vertical gap, can effectively increase $E(\Omega)$. One may question whether the increase in $E(\Omega)$ in these two cases above is purely due to the enlargement of the post (and thus shrinking of the vertical gap) or it does benefit from the changes in shape. To rule out the first possibility, we make up enlarged copies of the initial teardrop-shaped and nut-shaped posts, such that they have the same height (and thus the same vertical gaps between neighboring posts) as their corresponding optimized shapes. We plot the boundaries of enlarged posts in Figure 9 using solid curves, together with the corresponding optimized shapes that are plotted as dashed curves. We calculate $E(\Omega)$ for the enlarged posts. The enlarged teardrop-shaped post induces a normalized net flux $5.00 \times 10^{-2}$, and the enlarged 


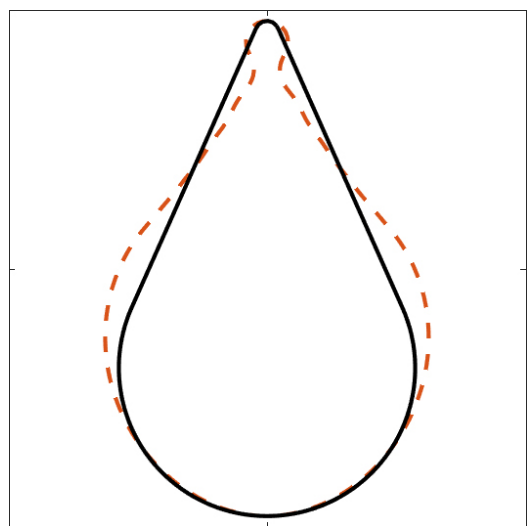

(a)

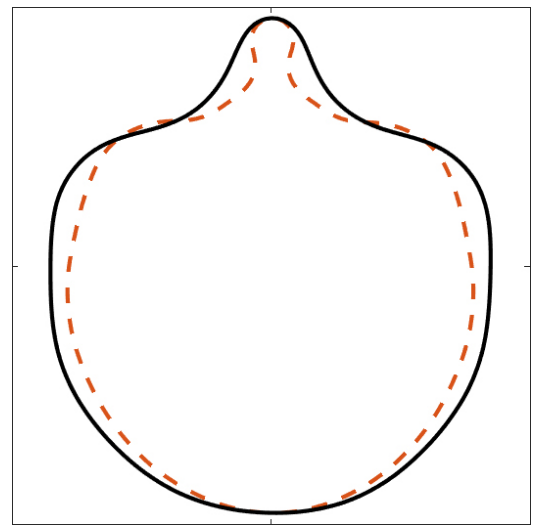

(b)

Figure 9: Enlarged posts with the initial shapes (solid curves) and posts with the optimized shapes (dashed curves). The heights of the enlarged posts are set to be the same as their corresponding optimized posts.

nut-shaped post gives $2.58 \times 10^{-2}$. In both cases, the enlarged posts can generate much larger net fluxes than the original ones, but still cannot compete with the optimized shapes. In this way, we justify that the shape optimization does help us find better designs of the post.

To summarize, in addition to the overall swelling of the post in the shape optimization, we empirically find different evolutions of the upper and lower halves of the post boundary. Round heads tend to form at the top of the posts with curvature there being in a narrow range $\kappa \in[24.5,26]$; a convex shape can evolve to become non-convex. By contrast, the lower half of the post always prefers to be a circular arc.

\section{An Approximate, but Informative, Analysis}

To better understand our findings and explore the possibility of designing posts with yet better shapes, we consider a simplified model. We divide the post boundary curve $\gamma$ into its upper and lower halves by cutting it at the left-most and the right-most points on $\gamma$. If there are several such points, pick the lowest one whenever necessary. See Figure 10 for an illustration. We denote the upper and lower halves of $\gamma$ by $\gamma_{U}$ and $\gamma_{L}$ respectively. Since $r_{\text {in }} \equiv 1$ on $\gamma$, when there is no a priori information about the spatial distribution of rods, then

$$
F_{U} \triangleq \int_{\gamma_{U}} r_{\text {out }}(\kappa(s)) d s
$$

becomes a good characterization of the capability of $\gamma_{U}$ releasing rods. Here $s$ is the arclength parameter of $\gamma$. Similarly,

$$
F_{L} \triangleq \int_{\gamma_{L}} r_{\text {out }}(\kappa(s)) d s
$$

is the corresponding quantity for $\gamma_{L}$. We naively assume that rods leaving from $\gamma_{U}$ are more likely to reach the top border of the unit cell than the bottom border, while rods released from $\gamma_{L}$ are more likely to cross the bottom border. By this assumption, the former type of rods contributes positively to $E(\Omega)$ since they cross the border in the positive $x_{2}$-direction, while the latter type contributes negatively. Hence, in a simplified manner, we use $F_{U}-F_{L}$ to characterize the overall capability of $\gamma$ inducing spontaneous migration in the positive $x_{2}$-direction. Here we ignore the variance in position 


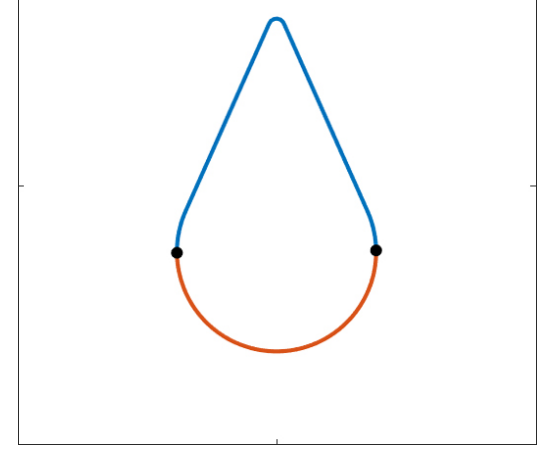

Figure 10: Dividing the post boundary $\gamma$ into its upper (blue) and lower (red) halves in the case of a teardrop-shaped post. The left- and right-most points on $\gamma$ are marked as black dots.

and orientation of rods when they cross either part of the boundary, and neither do we incorporate any information about the spatial distribution of rods. Therefore, to find a post with a good shape, we formally consider the following optimization problem,

$$
\max _{\gamma}\left(F_{U}-F_{L}\right)=\max _{\gamma}\left[\int_{\gamma_{U}} r_{\text {out }}(\kappa(s)) d s-\int_{\gamma_{L}} r_{\text {out }}(\kappa(s)) d s\right] .
$$

Here $r_{\text {out }}(\kappa)$ is given by (3.1) and we take maximum over all admissible curves $\gamma$. By admissible, we mean $\gamma$ that is sufficiently smooth and that does not intersect with itself. If needed, we may also impose a constraint that the curvature of $\gamma$ is bounded above and below by some constants.

To formally solve the maximization problem (5.1), we first note that

$$
\max _{\gamma}\left(F_{U}-F_{L}\right) \leq \max _{\gamma} \int_{\gamma_{U}} r_{\text {out }}(\kappa(s)) d s-\min _{\gamma^{\prime}} \int_{\gamma_{L}^{\prime}} r_{\text {out }}(\kappa(s)) d s .
$$

On the right hand side, the $\gamma$ that attains the maximum in the first term and the $\gamma^{\prime}$ that attains the minimum in the second term do not have to be the same one. The famous Gauss-Bonnet Theorem 38. will be useful in the following discussion, which states that for any admissible $\gamma$ in our context,

$$
\int_{\gamma_{U}} \kappa(s) d s=\int_{\gamma_{L}} \kappa(s) d s=\pi
$$

where $s$ is the arclength parameterizing $\gamma$ in a counter-clockwise orientation.

We first consider the maximizing problem in $(5.2)$. We rewrite

$$
\max _{\gamma} \int_{\gamma_{U}} r_{\text {out }}(\kappa(s)) d s=\max _{\gamma} \int_{\gamma_{U}} \frac{r_{\text {out }}(\kappa(s))}{\kappa(s)} \kappa(s) d s .
$$

Here $r_{\text {out }}(\kappa) / \kappa$ can be understood as the efficiency of utilizing the curvature to generate a desorption flux, given that (5.3) implies a fixed "budget" of the curvature on both $\gamma_{U}$ and $\gamma_{L}$. In order that the integral is maximized, $r_{\text {out }}(\kappa) / \kappa$ needs to be as large as possible when $\kappa>0$ and as small as possible when $\kappa<0$. It is known by (3.1) that $r_{\text {out }}(\kappa) / \kappa \rightarrow \pm \infty$ as $\kappa \rightarrow 0^{ \pm}$. Moreover, it is easy to show that $r_{\text {out }}(\kappa) / \kappa$ reaches a local maximum at $\kappa_{\max } \approx 25$. See Figure 11 . This implies that in the upper half of the post, the curvature tends to be close to 0 or $\kappa_{\max }$. This explains why the curvature at the heads in both examples above lies in a narrow range close to 25 instead of even larger values, such as $\kappa=65.1$ at the tip of the initial teardrop-shaped post. In addition, it does not harm to have negative curvature in $\gamma_{U}$ as it potentially increases the arclength where the $\kappa_{\max }$ 


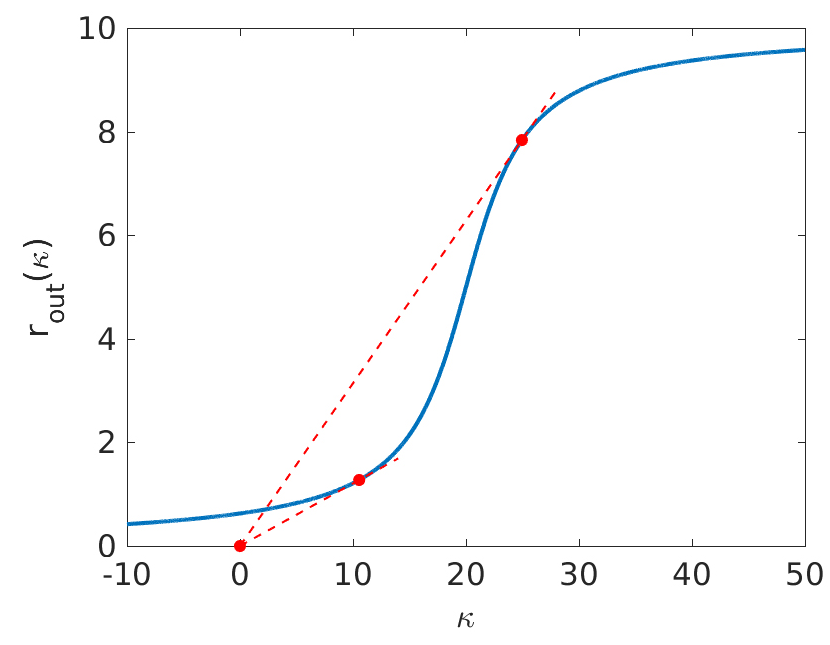

Figure 11: $r_{\text {out }}(\kappa) / \kappa$ reaches a local maximum at $\kappa_{\max } \approx 25$ and a local minimum at $\kappa_{\min } \approx 10.5$, marked as red dots on the curve. Note that $r_{\text {out }}(\kappa) / \kappa$ is the slope of straight line connecting the point $\left(\kappa, r_{\text {out }}(\kappa)\right)$ with the origin.

could be attained, thus improving the overall capability of $\gamma_{U}$ releasing rods. This explains why convex shapes can evolve into non-convex ones in the shape optimization.

Now we turn to the minimization problem involving $\gamma_{L}^{\prime}$ in $(5.2)$. We note that $r_{\text {out }}(\kappa) / \kappa$ has a local minimum at $\kappa_{\min } \approx 10.5$. See Figure 11 . If we rule out the case when $\kappa$ can be very large along $\gamma_{L}^{\prime}$, say assuming $\kappa<80$, we will find

$$
r_{\text {out }}(\kappa) \geq \frac{r_{\text {out }}\left(\kappa_{\text {min }}\right)}{\kappa_{\min }} \kappa, \quad \forall \kappa<80 .
$$

Hence,

$$
\min _{\gamma^{\prime}} \int_{\gamma_{L}^{\prime}} r_{\text {out }}(\kappa(s)) d s \geq \frac{r_{\text {out }}\left(\kappa_{\min }\right)}{\kappa_{\text {min }}} \int_{\gamma_{L}^{\prime}} \kappa(s) d s=\frac{\pi r_{\text {out }}\left(\kappa_{\text {min }}\right)}{\kappa_{\text {min }}} .
$$

Therefore, the minimum is achieved if $\gamma_{L}^{\prime}$ is a semicircle with dimensionless curvature $\kappa_{\min }$. This might not be obtained in general, because for example, the arclength between the left-most and the right-most points on $\gamma^{\prime}$ may not match the arclength of the semicircle with curvature $\kappa_{\min }$. In such case, $\gamma_{L}^{\prime}$ still has to be largely a semicircle. We can prove this under the assumption that $\kappa<20$ on $\gamma_{L}^{\prime}$, simply by noticing that $r_{\text {out }}(\kappa)$ is a convex function for $\kappa<20$ and then applying Jensen's inequality.

\section{Explorations of Other Designs}

In what follows, we shall design better posts based on the above simulations and analysis. We have seen that the round heads formed in Figure $7(\mathrm{a})$ and $8(\mathrm{a})$ act as strong desorption sites, which contribute a lot to increase $E(\Omega)$. It is natural to believe that $E(\Omega)$ can benefit from putting more strong desorption sites close to the top border of the unit cell. This inspires us to consider the new posts plotted in Figure 12(a) and 12(c) with multiple fingers at the top. Indeed, we choose these two shapes, such that the curvature at all fingertips satisfies $\kappa \in[23,26]$, presumably making them into strong desorption sites. Besides, we take the lower halves of these two shapes to be largely circular arcs. We compute $E(\Omega)$ for these two posts, without performing shape optimization. For the three-finger post, $E(\Omega)=4.78 \times 10^{-2}$; for the five-finger post, $E(\Omega)=6.18 \times 10^{-2}$, already close 
to our optimized single-head case. The spatial concentrations of the rods corresponding to these two candidate posts are also plotted in Figure 12(b) and Figure 12(d) respectively. It is clear that all the finger tips in both shapes are indeed strong desorption sites that are close to the top border of the unit cell. Efforts are being made to experimentally study the directed migration of swimmers induced by such posts with complex shapes.

Although these multi-finger posts already give strong net flux even without shape optimization, they still cannot compete the final optimized shape we obtained in Figure 7(a). One of the reasons is that there are still lots of open spaces between the post and the border of the unit cell. In particular, further reducing the vertical gap by enlarging the posts can hopefully lead to stronger fluxes. Another reason is the sub-optimality of their shapes. For example, the curvature of the lower half of these two posts are not close to $\kappa_{\min }$ found before. We also note that if we change the fingertips into small round heads with curvature close to $\kappa_{\max }$, the area of the strong desorption site can increase considerably and thus $E(\Omega)$ may increase as well.

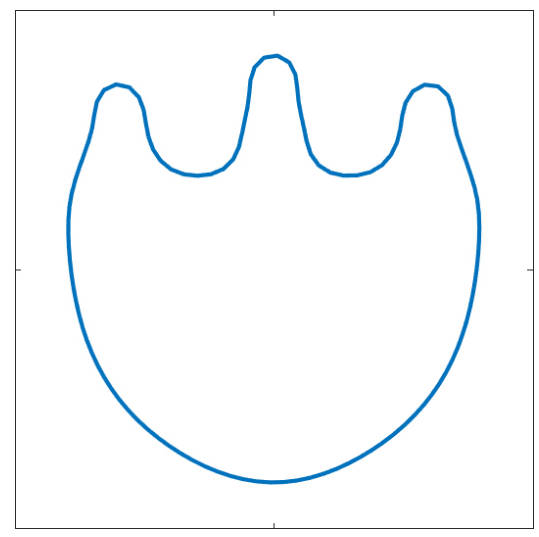

(a)

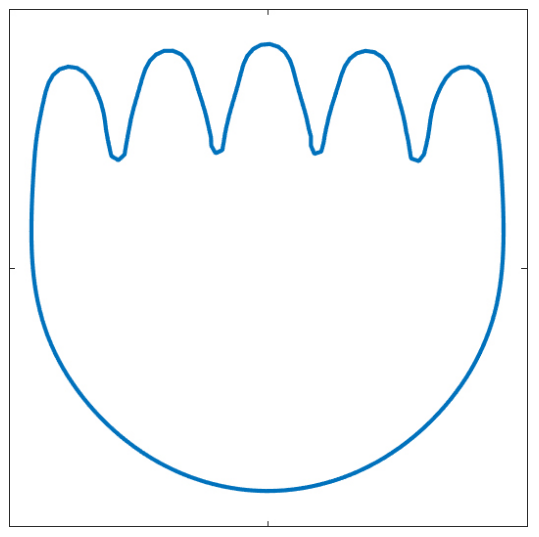

(c)

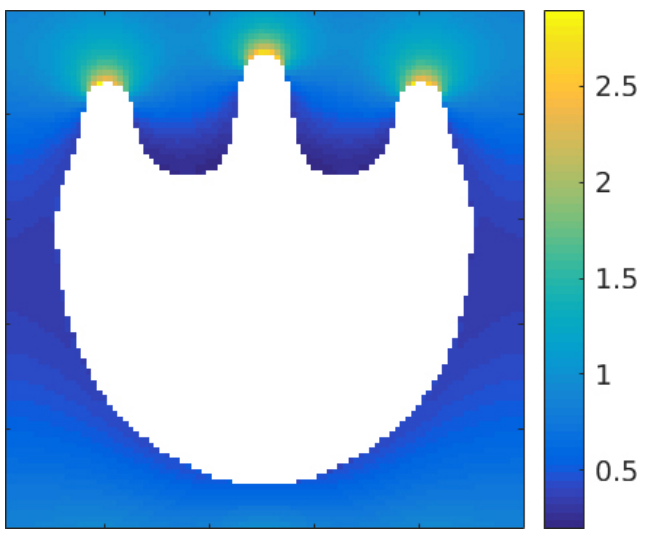

(b)

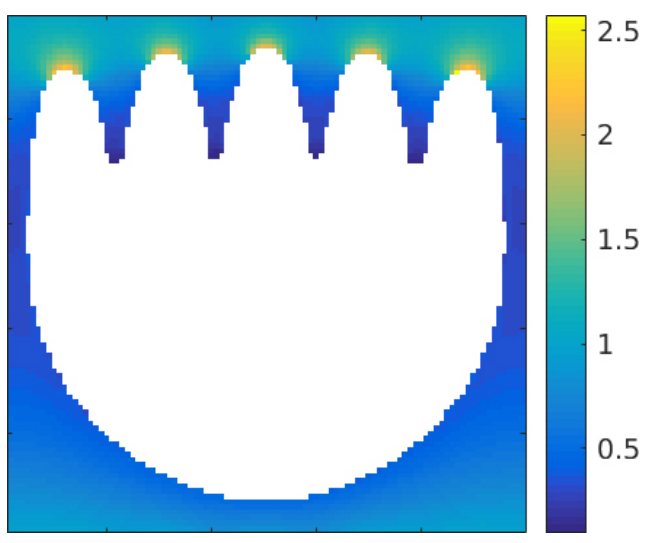

(d)

Figure 12: Two new candidate post shapes with multiple fingers at the top as strong desorption sites. All fingertips have curvature $\kappa \in[23,26]$ at the top, while the lower halves of the posts are designed to be largely circular arcs. (a-b) The three-finger post and its induced spatial rod concentration. In this case, $E(\Omega)=4.78 \times 10^{-2}$. (c-d) The five-finger post and its induced spatial rod concentration. In this case, $E(\Omega)=6.18 \times 10^{-2}$.

Now we propose a shape that is almost the optimal in the sense that: it leaves very little open space for the free swimming of the rods; and it almost solves the simplified optimization problem on 
the right hand side of 5.2 by putting the right curvatures in the upper and lower halves of $\gamma$. We plot it in Figure 13 using blue curves in a $2 \times 5$-array. The dashed box represents one unit cell of the array, with $a=0.2$ and $b=1$. The post in it has a slim shape, whose height and width are 0.940 and 0.180 respectively. It has flat sides and curved top and bottom parts. The top part consists of two semi-circular arcs with $\kappa \approx 23$, which is close to $\kappa_{\max } \approx 25$; the bottom part is a semi-circular arc, with $\kappa \approx 11$, which is close to $\kappa_{\min } \approx 10.5$. There are only narrow gaps between neighboring posts. We compute its normalized net flux to obtain $E(\Omega)=1.15 \times 10^{-1}$, which surpasses any other posts considered in this paper! Note that we do not rule out the existence of even more judicious designs of posts. In sum, we showed that using what we have learned in the numerical shape optimization and the formal analysis above, we can design better shapes of posts effectively.

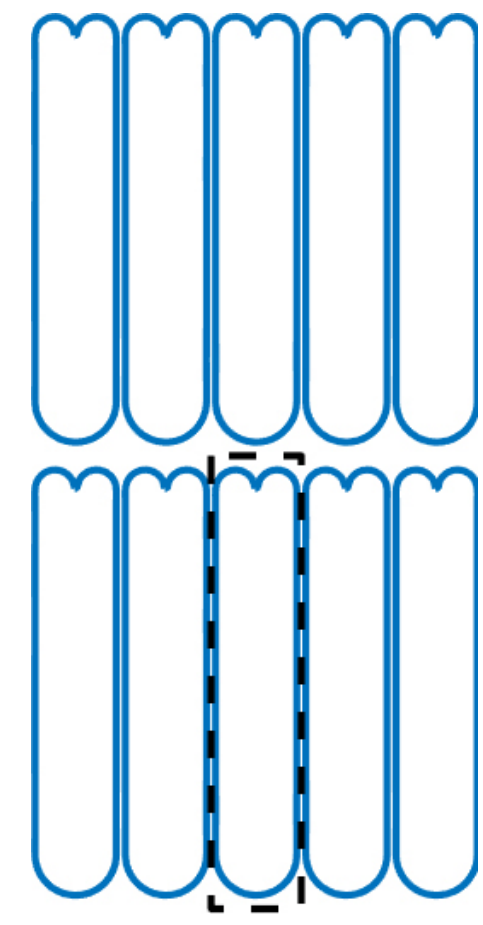

Figure 13: A $2 \times 5$-array of a slim post. Its unit cell has $a=0.2$ and $b=1$. The post has flat sides and curved top and bottom parts. The top part consists of two semi-circles with $\kappa \approx 23$, while the bottom part is a semi-circle with $\kappa \approx 11$. There are only narrow gaps between neighboring posts in the same row. Such an array induces a normalized net flux $E(\Omega)=1.15 \times 10^{-1}$.

\section{Conclusion and Discussion}

In this paper, we propose a kinetic-type model to study $\mathrm{Au}-\mathrm{Pt}$ rods swimming and directionally migrating in a periodic array of posts with non-circular cross-sections. Both position and orientation of the rods are taken into account. The absorption and desorption of the swimming rods on the post boundaries are modeled via empirically defined rate functions of the boundary curvature, and angular distributions. Within this model, we define and compute the normalized net flux induced by a periodic rectangular array of posts, which characterizes the intensity of the spontaneous directed migration of rods in the array due to the asymmetry in geometry. We study how to design the array judiciously so that it can induce stronger directed migration. It is shown that the net flux increases if the horizontal and vertical spacings between neighboring posts shrink. On the other hand, we apply 
the numerical shape optimization to find better shapes of posts that induce yet larger flux. Inspired by the numerical results on two candidate posts - a teardrop-shaped post and a nut-shaped post, we propose a simplified model to show the key geometric features a good post should have. Based on that, we come up with three new candidate shapes that generate large fluxes. In this way, we show the shape optimization technique can help design good posts effectively.

Our results crucially rely on the choices of (hopefully reasonable) universal rate functions $r_{\text {in }}(\kappa)$ and $r_{\text {out }}(\kappa)$, and the angular distributions $\rho_{ \pm}(\beta)$ and $\tau_{ \pm}(\beta)$, for which little experimental measurement exists. In this paper, we choose these functions so that they qualitatively agree with the existing experimental observations and physical intuition. We note that our choices of $\rho_{ \pm}(\beta)$ and $\tau_{ \pm}(\beta)$ are natural and reasonable. The numerical experience is that, even if we alter the choice of these angular distributions, the numerical results are qualitatively unchanged. By contrast, $r_{\text {in }}(\kappa)$ and $r_{\text {out }}(\kappa)$ could have bigger impacts on the numerical results. It has been shown in the simplified model in Section 4 that given $r_{\text {in }} \equiv 1$, the local maximum and minimum of $r_{\text {out }}(\kappa) / \kappa$ can be very crucial quantities that determine geometric features of a good post. For example, if we alternatively take $r_{\text {out }}(\kappa)$ to be $e^{\kappa}$, one would expect a good post to have very sharp tips at its top instead of small round heads or circular arcs, since $r_{\text {out }}(\kappa) / \kappa$ formally achieves maximum at $\kappa=+\infty$. If we further choose $r_{\text {in }}(\kappa)$ to be a function depending on $\kappa$, the situation would be more complicated and it can hardly be characterized by our simplified model.

In the current model, the absorption and desorption of the swimming rods on the post boundary are handled in a phenomenological manner, which is the reason we need the functions above. It would be ideal to build up a hydrodynamic model to fully characterize the interaction between the swimming bimetallic rods and the curved solid boundary, from which we can hopefully derive the rate functions and angular distributions that are needed. Previous work by Takagi et al. 20] applying lubrication theory between a swimming rod and a flat solid boundary cannot be immediately generalized to the case of the curved boundary, especially when the radius of curvature of the boundary is on the same scale as or even below the rod length, which is the case when a rod comes to the sharp tip of a teardrop-shaped post. Spagnolie et al. [15] models hydrodynamic capture and escape of microswimmers on an obstacle, by assuming the swimmers to be force dipoles. However, this analysis assumes that the swimmer can preserve its orientation when it hits the boundary, which is not true in our case. More delicate modeling is thus needed to understand the hydrodynamic interaction between swimmers and complex boundaries in fluid environments.

\section{Acknowledgement}

We want to thank our colleagues in Courant Institute and Department of Chemistry of New York University, Dr. Megan S. Davies Wykes, Dr. Xiao Zhong, Prof. Leif Ristroph, Prof. Jun Zhang, Prof. Michael D. Ward, Prof. Yanpeng Liu, Jinzi Mac Huang, Yang Wu and Dr. Abtin Rahimian, for many inspiring discussions and providing useful experimental data. The project is partially supported by NSF under Grant DMS-1463962.

\section{References}

[1] Jens Elgeti, Roland G Winkler, and Gerhard Gompper. Physics of microswimmers: single particle motion and collective behavior: a review. Reports on Progress in Physics, 78(5):056601, 2015 .

[2] Stephen J Ebbens and Jonathan R Howse. In pursuit of propulsion at the nanoscale. Soft Matter, 6(4):726-738, 2010. 
[3] Debabrata Patra, Samudra Sengupta, Wentao Duan, Hua Zhang, Ryan Pavlick, and Ayusman Sen. Intelligent, self-powered, drug delivery systems. Nanoscale, 5(4):1273-1283, 2013.

[4] Shankar Balasubramanian, Daniel Kagan, Che-Ming Jack Hu, Susana Campuzano, M Jesus Lobo-Castañon, Nicole Lim, Dae Y Kang, Maria Zimmerman, Liangfang Zhang, and Joseph Wang. Micromachine-enabled capture and isolation of cancer cells in complex media. Angewandte Chemie International Edition, 50(18):4161-4164, 2011.

[5] Shakuntala Sundararajan, Paul E Lammert, Andrew W Zudans, Vincent H Crespi, and Ayusman Sen. Catalytic motors for transport of colloidal cargo. Nano Letters, 8(5):1271-1276, 2008.

[6] Lluís Soler, Veronika Magdanz, Vladimir M Fomin, Samuel Sanchez, and Oliver G Schmidt. Self-propelled micromotors for cleaning polluted water. ACS Nano, 7(11):9611-9620, 2013.

[7] Jonathan R Howse, Richard AL Jones, Anthony J Ryan, Tim Gough, Reza Vafabakhsh, and Ramin Golestanian. Self-motile colloidal particles: from directed propulsion to random walk. Physical Review Letters, 99(4):048102, 2007.

[8] Jérémie Palacci, Cécile Cottin-Bizonne, Christophe Ybert, and Lydéric Bocquet. Sedimentation and effective temperature of active colloidal suspensions. Physical Review Letters, 105(8):088304, 2010.

[9] Howard C Berg, Douglas A Brown, et al. Chemotaxis in Escherichia coli analysed by threedimensional tracking. Nature, 239(5374):500-504, 1972.

[10] James G Mitchell and Kazuhiro Kogure. Bacterial motility: links to the environment and a driving force for microbial physics. FEMS Microbiology Ecology, 55(1):3-16, 2006.

[11] John L Anderson. Colloid transport by interfacial forces. Annual Review of Fluid Mechanics, 21(1):61-99, 1989.

[12] Ambarish Ghosh and Peer Fischer. Controlled propulsion of artificial magnetic nanostructured propellers. Nano Letters, 9(6):2243-2245, 2009.

[13] Giovanni Volpe, Ivo Buttinoni, Dominik Vogt, Hans-Jürgen Kümmerer, and Clemens Bechinger. Microswimmers in patterned environments. Soft Matter, 7(19):8810-8815, 2011.

[14] Pietro Tierno, Ramin Golestanian, Ignacio Pagonabarraga, and Francesc Sagués. Magnetically actuated colloidal microswimmers. The Journal of Physical Chemistry B, 112(51):16525-16528, 2008 .

[15] Saverio E Spagnolie, Gregorio R Moreno-Flores, Denis Bartolo, and Eric Lauga. Geometric capture and escape of a microswimmer colliding with an obstacle. Soft Matter, 11(17):3396$3411,2015$.

[16] Vasily Kantsler, Jörn Dunkel, Marco Polin, and Raymond E Goldstein. Ciliary contact interactions dominate surface scattering of swimming eukaryotes. Proceedings of the National Academy of Sciences, 110(4):1187-1192, 2013.

[17] Guanglai Li, James Bensson, Liana Nisimova, Daniel Munger, Panrapee Mahautmr, Jay X Tang, Martin R Maxey, and Yves V Brun. Accumulation of swimming bacteria near a solid surface. Physical Review E, 84(4):041932, 2011.

[18] Guanglai Li and Jay X Tang. Accumulation of microswimmers near a surface mediated by collision and rotational brownian motion. Physical Review Letters, 103(7):078101, 2009. 
[19] Allison P Berke, Linda Turner, Howard C Berg, and Eric Lauga. Hydrodynamic attraction of swimming microorganisms by surfaces. Physical Review Letters, 101(3):038102, 2008.

[20] Daisuke Takagi, Jérémie Palacci, Adam B Braunschweig, Michael J Shelley, and Jun Zhang. Hydrodynamic capture of microswimmers into sphere-bound orbits. Soft Matter, 10(11):1784$1789,2014$.

[21] WE Uspal, Mikhail N Popescu, S Dietrich, and M Tasinkevych. Self-propulsion of a catalytically active particle near a planar wall: from reflection to sliding and hovering. Soft Matter, 11(3):434438, 2015.

[22] Luis Cisneros, Christopher Dombrowski, Raymond E Goldstein, and John O Kessler. Reversal of bacterial locomotion at an obstacle. Physical Review E, 73(3):030901, 2006.

[23] Peter Galajda, Juan Keymer, Paul Chaikin, and Robert Austin. A wall of funnels concentrates swimming bacteria. Journal of Bacteriology, 189(23):8704-8707, 2007.

[24] Guillaume Lambert, David Liao, and Robert H Austin. Collective escape of chemotactic swimmers through microscopic ratchets. Physical Review Letters, 104(16):168102, 2010.

[25] Juliane Simmchen, Jaideep Katuri, William E Uspal, Mihail N Popescu, Mykola Tasinkevych, and Samuel Sánchez. Topographical pathways guide chemical microswimmers. Nature Communications, 7, 2016.

[26] Megan S Davies Wykes, Xiao Zhong, Jiajun Tong, Takuji Adachi, Yanpeng Liu, Leif Ristroph, Michael D Ward, Michael J Shelley, and Jun Zhang. Guiding microscale swimmers using teardrop-shaped posts. Soft Matter, 2017.

[27] Walter F Paxton, Kevin C Kistler, Christine C Olmeda, Ayusman Sen, Sarah K St. Angelo, Yanyan Cao, Thomas E Mallouk, Paul E Lammert, and Vincent H Crespi. Catalytic nanomotors: autonomous movement of striped nanorods. Journal of the American Chemical Society, 126(41):13424-13431, 2004.

[28] Jeffrey L Moran and Jonathan D Posner. Electrokinetic locomotion due to reaction-induced charge auto-electrophoresis. Journal of Fluid Mechanics, 680:31-66, 2011.

[29] Yang Wang, Rose M Hernandez, David J Bartlett, Julia M Bingham, Timothy R Kline, Ayusman Sen, and Thomas E Mallouk. Bipolar electrochemical mechanism for the propulsion of catalytic nanomotors in hydrogen peroxide solutions. Langmuir, 22(25):10451-10456, 2006.

[30] Jan Sokolowski and Jean-Paul Zolesio. Introduction to shape optimization. Springer, Berlin, Heidelberg, 1992.

[31] Michel C Delfour and J-P Zolésio. Shapes and geometries: metrics, analysis, differential calculus, and optimization, volume 22. Siam, 2011.

[32] Olivier Pironneau. Optimal shape design for elliptic systems. Springer Science \& Business Media, 2012.

[33] Eric E Keaveny, Shawn W Walker, and Michael J Shelley. Optimization of chiral structures for microscale propulsion. Nano Letters, 13(2):531-537, 2013.

[34] Jon Wilkening and AE Hosoi. Shape optimization of a sheet swimming over a thin liquid layer. Journal of Fluid Mechanics, 601:25-61, 2008. 
[35] Shawn W Walker and Michael J Shelley. Shape optimization of peristaltic pumping. Journal of Computational Physics, 229(4):1260-1291, 2010.

[36] Bijan Mohammadi and Olivier Pironneau. Applied shape optimization for fluids. Oxford university press, 2010.

[37] Chang Liu, Chao Zhou, Wei Wang, and H. P. Zhang. Bimetallic microswimmers speed up in confining channels. Physical Review Letters, 117:198001, Nov 2016.

[38] Manfredo Perdigao Do Carmo. Differential geometry of curves and surfaces, volume 2. Prenticehall Englewood Cliffs, 1976.

[39] David Gilbarg and Neil S Trudinger. Elliptic partial differential equations of second order. Springer, 2015.

[40] COMSOL Multiphysics. LiveLink for MATLAB®. User's guide, Version, 4.

[41] Gene H Golub and Charles F Van Loan. Matrix computations, volume 3. JHU Press, 2012. 


\section{Supplementary Materials}

The Supplementary Materials contain all the numerical methods and mathematical derivations omitted in the main body of the paper. It is organized as follows. In Section A, we discuss the numerical methods of solving the model (2.7)-(2.11) for $p$ and $p_{B}^{ \pm}$under the normalization condition 2.12 and calculating the corresponding flux $E(\Omega)$. Section $B$ is a quick overview of the theory of shape optimization, which is the mathematical foundation of the derivation in Section C, D and E. In Section C we derive the equations for the shape derivatives $p^{\prime}$ and $p_{B}^{ \pm \prime}$, whose meanings will be clear in Section B. In Section D, we rewrite the Eulerian derivative of the shape functional $E(\Omega)$ with respect to the perturbation of the shape of the post into an integral on the boundary that is being perturbed, before which we define several auxiliary functions. Section E is used to justify the linear dependence of the Eularian derivative on the normal component of the perturbation vector field. The numerical method to evolve and optimize the boundary curve of the post is discussed in Section $\mathrm{F}$.

\section{A Numerical methods of solving the model (2.7)-(2.11) and calculating $E(\Omega)$ in the static geometry}

We start with the following formal analysis based on the model (2.7)-(2.11). Given $p_{B}^{ \pm}(x)$ as functions on $\gamma$, define

$$
g(x, \theta)=r_{\text {out }}(\kappa(x))\left[2 \pi \tau_{+}(\beta) p_{B}^{+}(x)+2 \pi \tau_{-}(\beta) p_{B}^{-}(x)\right], \quad(x, \theta) \in \Gamma .
$$

We denote $g(x, \theta)=g\left[p_{B}^{+}, p_{B}^{-}\right]$, where $g[\cdot]$ is obviously linear in $\left(p_{B}^{+}, p_{B}^{-}\right)$. Given this $g(x, \theta)$ on $\Gamma$, we solve the following sub-problem adapted from (2.7) and (2.9)-(2.11),

$$
\begin{aligned}
& D_{t} \Delta_{x} \tilde{p}(x, \theta)+D_{r} \Delta_{\theta} \tilde{p}(x, \theta)-v_{0}(\cos 2 \pi \theta, \sin 2 \pi \theta)^{T} \cdot \nabla_{x} \tilde{p}(x, \theta)=0, \quad(x, \theta) \in \Omega, \\
& D_{t} \frac{\partial \tilde{p}}{\partial n_{\Gamma}}(x, \theta)-v_{0} \tilde{p}(x, \theta) \cos \beta+r_{\text {in }}(\kappa(x))\left(\rho_{+}(\beta)+\rho_{-}(\beta)\right) \tilde{p}(x, \theta)=g(x, \theta), \quad(x, \theta) \in \Gamma, \\
& \tilde{p} \text { satisfies periodic boundary condition on } \partial \Omega \backslash \Gamma,
\end{aligned}
$$

where $\beta=2 \pi \theta-\alpha(x)$. Suppose the equation is solvable for any $g(x, \theta)$. The solution is denoted by $\tilde{p}(x, \theta)$. The linear map from $g$ to $\left.\tilde{p}\right|_{\Gamma}$ is denoted to be $T$, i.e. $\left.\tilde{p}\right|_{\Gamma} \triangleq T g$.

With the $\left.\tilde{p}\right|_{\Gamma}$ in hand, we solve the following equation for $\tilde{p}_{B}^{ \pm}$on $\gamma$ adapted from $(2.8)$ and (2.11),

$$
-D_{t} \Delta_{\gamma} \tilde{p}_{B}^{ \pm}(x) \pm v_{0} \partial_{\gamma} \tilde{p}_{B}^{ \pm}(x)+r_{\text {out }}(\kappa(x)) \tilde{p}_{B}^{ \pm}(x)=\left.\int_{0}^{1} r_{\text {in }}(\kappa(x)) \rho_{ \pm}(\beta) \tilde{p}\right|_{\Gamma}(x, \theta) \mathrm{d} \theta, \quad x \in \gamma .
$$

Here we used the assumption that $\tau_{ \pm}$are normalized. This equation for $p_{B}^{ \pm}$is always solvable as long as $r_{\text {out }}(\kappa(x))$ is positive and sufficiently smooth. We denote that $\tilde{p}_{B}^{ \pm}=Q^{ \pm} T g$. Here $Q^{ \pm}$are also linear maps.

To this end, we find that

$$
\left(\tilde{p}_{B}^{+}, \tilde{p}_{B}^{-}\right)=\left(Q^{+} T g\left[p_{B}^{+}, p_{B}^{-}\right], Q^{-} T g\left[p_{B}^{+}, p_{B}^{-}\right]\right) \triangleq K\left[p_{B}^{+}, p_{B}^{-}\right],
$$

where $K$ maps $\left[p_{B}^{+}, p_{B}^{-}\right]$linearly to $\left[\tilde{p}_{B}^{+}, \tilde{p}_{B}^{-}\right]$. Now solving the model 2.7)-2.11 reduces to solving the following fixed point problem

$$
K\left[p_{B}^{+}, p_{B}^{-}\right]=\left(p_{B}^{+}, p_{B}^{-}\right),
$$

i.e. the desired solution $\left(p_{B}^{+}, p_{B}^{-}\right)$should be in the kernel of the linear operator $(K-I d)$. Once such $\left(p_{B}^{+}, p_{B}^{-}\right)$is found out, $p$ could be solved by first using A.1 to calculate $g$, and then solving the subproblem A.2 - A.4. We may have to normalize $p$ and $p_{B}^{ \pm}$so that 2.12 is satisfied. The normalized net flux $E(\Omega)$ is then calculated by $(2.13)$. 
Remark A.1. There exists a unique positive smooth solution $\left(p, p_{B}^{+}, p_{B}^{-}\right)$of the model 2.7 -2.11 satisfying [2.12), provided that

1. $\gamma$ is sufficiently smooth, and thus $\alpha(x)$ and $\kappa(x)$ are sufficiently smooth in $x \in \gamma$;

2. $r_{\text {in }}(\kappa)>0, r_{\text {out }}(\kappa)>0, \rho_{ \pm}(\beta)$ and $\tau_{ \pm}(\beta)$, where $\kappa=\kappa(x)$ and $\beta=2 \pi \theta-\alpha(x)$, are sufficiently smooth in $(x, \theta) \in \Gamma$;

3. and in addition, $r_{\text {in }}(\kappa(x))\left(\rho_{+}(\beta)+\rho_{-}(\beta)\right)-v_{0} \cos \beta \geq 0$ but not identically zero.

This could be proved by applying elliptic regularity theory to study the operator $K$ and using the Schauder fixed point theorem [39]. We shall omit the proof. In what follows, we always assume $(K-I d)$ has a one-dimensional kernel.

Remark A.2. It could be shown that $\left(r_{\text {out }}(x), r_{\text {out }}(x)\right)$ is orthogonal to the image of $(K-I d)$ in the inner product on $L^{2}(\gamma) \times L^{2}(\gamma)$. Indeed, by A.1 - A.5,

$$
\begin{aligned}
\int_{\gamma} r_{\text {out }}\left(\tilde{p}_{B}^{+}+\tilde{p}_{B}^{-}\right) & =\int_{\Gamma} D_{t} \Delta_{\gamma}\left(\tilde{p}_{B}^{+}+\tilde{p}_{B}^{-}\right)-v_{0} \partial_{\gamma}\left(\tilde{p}_{B}^{+}-\tilde{p}_{B}^{-}\right)+r_{\text {in }}\left(\rho_{+}+\rho_{-}\right) \tilde{p} \\
& =\int_{\Gamma} r_{\text {in }}\left(\rho_{+}+\rho_{-}\right) \tilde{p}=\int_{\Gamma} g(x, \theta)-D_{t} \frac{\partial \tilde{p}}{\partial n_{\Gamma}}+v_{0} \tilde{p} \cos \beta \\
& =\int_{\Gamma} g(x, \theta)-\int_{\Omega} D_{t} \Delta_{x} \tilde{p}+D_{r} \Delta_{\theta} \tilde{p}-v_{0}(\cos 2 \pi \theta, \sin 2 \pi \theta)^{T} \cdot \nabla_{x} \tilde{p} \\
& =\int_{\Gamma} r_{\text {out }}\left(2 \pi \tau_{+} p_{B}^{+}+2 \pi \tau_{-} p_{B}^{-}\right)=\int_{\gamma} r_{\text {out }}\left(p_{B}^{+}+p_{B}^{-}\right) .
\end{aligned}
$$

Therefore, we find for $\forall\left(p_{B}^{+}, p_{B}^{-}\right)$,

$$
\left\langle\left(r_{\text {out }}, r_{\text {out }}\right),(K-I d)\left[p_{B}^{+}, p_{B}^{-}\right]\right\rangle=0,
$$

where $\langle\cdot, \cdot\rangle$ denotes the inner product on $L^{2}(\gamma) \times L^{2}(\gamma)$. This property is preserved, up to small numerical error, when A.1)-A.5 are discretized and solved numerically. This indicates that the matrix representation of $(K-I d)$ in the discretized case should have one (and exactly one due to the assumption in Remark A.1 zero singular value, up to numerical error.

Based on the above analysis, the numerical method to solve the model should go as follows. It aims at forming a discrete representation of the operator $(K-I d)$, finding out its null space, and calculating $E(\Omega)$.

1. With abuse of notations $x_{1}$ and $x_{2}$, we represent $\gamma$ by $N$ points $\left\{x_{1}, \cdots, x_{N}\right\} \triangleq X \subset \gamma$, which are equally aligned on $\gamma$ counterclockwise with spacing $\Delta s$, i.e. the arclength of $\gamma$ between two neighboring points are assumed to be $\Delta s$. Here $N$ is assumed to be even. We use central difference scheme to evaluate $\alpha\left(x_{j}\right), n\left(x_{j}\right)$ and $\kappa\left(x_{j}\right)$ for $j=1,2, \cdots, N$, i.e. take $\alpha\left(x_{j}\right)$ such that

$$
\left(\cos \alpha\left(x_{j}\right), \sin \alpha\left(x_{j}\right)\right)=\frac{x_{j+1}-x_{j-1}}{\left|x_{j+1}-x_{j-1}\right|} .
$$

where the subscripts are understood in a cyclic fashion. Then

$$
\begin{aligned}
& n\left(x_{j}\right)=\left(-\sin \alpha\left(x_{j}\right), \cos \alpha\left(x_{j}\right)\right), \\
& \kappa\left(x_{j}\right)=\frac{1}{2 \Delta s}\left[\left(\cos \alpha\left(x_{j+1}\right), \sin \alpha\left(x_{j+1}\right)\right)-\left(\cos \alpha\left(x_{j-1}\right), \sin \alpha\left(x_{j-1}\right)\right)\right] \cdot n\left(x_{j}\right) .
\end{aligned}
$$

In the $\theta$-direction, $[0,1]$ is discretized evenly using $M$ points $\theta_{m}=\frac{2 m-1}{2 M}$ with $m=1,2, \cdots, M$. Hence, the grid on $\Gamma$ is given by

$$
X_{\Gamma} \triangleq X \times\left\{\theta_{1}, \theta_{2}, \cdots, \theta_{M}\right\}=\left\{\left(x_{j}, \theta_{m}\right): j=1,2, \cdots, N, m=1,2, \cdots, M\right\} .
$$


2. Let $\left\{\mathbf{u}_{1}, \cdots, \mathbf{u}_{2 N}\right\}$ be an orthogonal basis of $\mathbb{R}^{2 N} ; \mathbf{u}_{i}$ 's are understood as column vectors. Denote $U=\left(\mathbf{u}_{1}, \cdots, \mathbf{u}_{2 N}\right)$. We shall use Fourier modes to generate $\mathbf{u}_{i}$ 's. To be more precise, let $U_{0}$ be an $N \times N$-matrix, whose $(i, j)$-entry is given by

$$
\begin{aligned}
& U_{0, i j}=\sin (2 \pi i j / N), \quad i \in\{1, \cdots, N / 2-1\}, j \in\{1, \cdots, N\}, \\
& U_{0, i j}=\cos (2 \pi(i-N / 2) j / N), \quad i \in\{N / 2, \cdots, N\}, j \in\{1, \cdots, N\} .
\end{aligned}
$$

Note that the rows of $U_{0}$ form an orthgonal basis of $\mathbb{R}^{N}$. Then we define

$$
U=\left(\begin{array}{cc}
U_{0}^{T} & 0 \\
0 & U_{0}^{T}
\end{array}\right)_{2 N \times 2 N}=\left(\mathbf{u}_{1}, \cdots, \mathbf{u}_{2 N}\right) .
$$

$\left\{\mathbf{u}_{1}, \cdots, \mathbf{u}_{2 N}\right\}$ form a basis of all possible vectorial representations of $\left(p_{B}^{+}, p_{B}^{-}\right)^{T}$ on the grid points $x_{n}$ 's.

3. Put

$$
\left(p_{B, i}^{+}\left(x_{1}\right), \cdots, p_{B, i}^{+}\left(x_{N}\right), p_{B, i}^{-}\left(x_{1}\right), \cdots, p_{B, i}^{-}\left(x_{N}\right)\right)^{T}=\mathbf{u}_{i} .
$$

For $j=1,2, \cdots, N$ and $m=1,2, \cdots, M$, calculate

$$
g_{i}\left(x_{j}, \theta_{m}\right)=r_{\text {out }}\left(\kappa\left(x_{j}\right)\right)\left[2 \pi \tau_{+}\left(\beta\left(x_{j}, \theta_{m}\right)\right) p_{B, i}^{+}\left(x_{j}\right)+2 \pi \tau_{-}\left(\beta\left(x_{j}, \theta_{m}\right)\right) p_{B, i}^{-}\left(x_{j}\right)\right],
$$

where $\beta\left(x_{j}, \theta_{m}\right)=\alpha\left(x_{j}\right)-2 \pi \theta_{m}$. With $g_{i}$ well-defined on $X_{\Gamma}$, we use the commercial finite element package COMSOL Multiphysics [40] to solve the following subproblem in dimension three.

$$
\begin{aligned}
& D_{t} \Delta_{x} \tilde{p}_{i}(x, \theta)+D_{r} \Delta_{\theta} \tilde{p}_{i}(x, \theta)-v_{0}(\cos 2 \pi \theta, \sin 2 \pi \theta)^{T} \cdot \nabla_{x} \tilde{p}_{i}(x, \theta)=0, \quad(x, \theta) \in \Omega, \\
& D_{t} \frac{\partial \tilde{p}_{i}}{\partial n_{\Gamma}}(x, \theta)-v_{0} \tilde{p}_{i}(x, \theta) \cos \beta+r_{\mathrm{in}}(\kappa(x))\left(\rho_{+}(\beta)+\rho_{-}(\beta)\right) \tilde{p}_{i}(x, \theta)=g_{i}(x, \theta), \quad(x, \theta) \in \Gamma,
\end{aligned}
$$

$\tilde{p}_{i}$ satisfies periodic boundary condition on $\partial \Omega \backslash \Gamma$.

We obtain the Dirichlet boundary data of $\tilde{p}_{i}$ on $X_{\Gamma}$.

4. Now consider the equation for $\tilde{p}_{B, i}^{ \pm}$

$$
-D_{t} \Delta_{\gamma} \tilde{p}_{B, i}^{ \pm}(x) \pm v_{0} \partial_{\gamma} \tilde{p}_{B, i}^{ \pm}(x)+r_{\text {out }}(\kappa(x)) \tilde{p}_{B, i}^{ \pm}(x)=\left.\int_{0}^{1} r_{\text {in }}(\kappa(x)) \rho_{ \pm}(\beta) \tilde{p}_{i}\right|_{\Gamma}(x, \theta) \mathrm{d} \theta, \quad x \in \gamma .
$$

Here the integral is evaluated using the trapezoidal rule:

$$
\left.\left[\left.\int_{0}^{1} r_{\mathrm{in}}(\kappa(x)) \rho_{ \pm}(\beta) \tilde{p}_{i}\right|_{\Gamma}(x, \theta) \mathrm{d} \theta\right]\right|_{x=x_{j}}=\frac{1}{M} \sum_{m=1}^{M} r_{\mathrm{in}}\left(\kappa\left(x_{j}\right)\right) \rho_{ \pm}\left(\beta\left(x_{j}, \theta_{m}\right)\right) \tilde{p}_{i}\left(x_{j}, \theta_{m}\right) .
$$

By virtue of the periodic boundary condition in the $\theta$-direction, the trapezoidal rule can achieve high accuracy even when $M$ is not very large. Then we solve A.16 for $\tilde{p}_{B, i}^{ \pm}$using the usual finite difference scheme. Denote

$$
\left(\tilde{p}_{B, i}^{+}\left(x_{1}\right), \cdots, \tilde{p}_{B, i}^{+}\left(x_{N}\right), \tilde{p}_{B, i}^{-}\left(x_{1}\right), \cdots, \tilde{p}_{B, i}^{-}\left(x_{N}\right)\right)^{T} \triangleq \mathbf{w}_{i} \in \mathbb{R}^{2 N} .
$$

In this way, we obtain $\mathbf{w}_{i}=K \mathbf{u}_{i}$, where $K$, with abuse of notations, is a discrete representation of the operator $K$ defined in A.6. It is not explicitly defined at this moment. 
5. Going through Step 3 and 4 for $i=1,2, \cdots, 2 N$, we obtain a $2 N \times 2 N$ matrix

$$
W \triangleq\left(\mathbf{w}_{1}, \cdots, \mathbf{w}_{2 N}\right)=K U \text {. }
$$

Now it suffices to find out an eigenvector of $K-I_{2 N}$ corresponding to zero eigenvalue. Assume it can be represented by $U \xi$ for some $\xi \in \mathbb{R}^{2 N}$. Hence, $(W-U) \xi=\left(K-I_{2 N}\right) U \xi=0$. By singular value decomposition, we can find out the zero eigenvector of $W-U$, or equivalently the right singular vector corresponding to zero singular value, denoted by $\xi_{*}$. Then values of the unnormalized solution $p_{B}^{ \pm}$on $X$ are given by

$$
\left(p_{B}^{+}\left(x_{1}\right), \cdots, p_{B}^{+}\left(x_{N}\right), p_{B}^{-}\left(x_{1}\right), \cdots, p_{B}^{-}\left(x_{N}\right)\right)^{T}=U \xi_{*} .
$$

6. We go through Step 3 again with (A.15) replaced by (A.18) and $\left(\tilde{p}_{i}, p_{B, i}^{ \pm}\right)$replaced by $\left(p, p_{B}^{ \pm}\right)$to find out the (unnormalized) solution $p$ in $\Omega$. Normalize $p$ and $p_{B}^{ \pm}$such that 2.12 is satisfied. Finally, we calculate $E(\Omega)$ by 2.13 using trapezoidal rule. Note that we can obtain all the necessary boundary data from COMSOL and COMSOL can calculate $\int_{\Omega} p d x d \theta$ automatically.

Remark A.3. In practice, the matrix $W-U$ does not necessarily have a zero singular value due to numerical errors. In typical numerical experiments, we always find that one of the singular values of $W-U$ is very close to 0 , while the rest are all of order 1 . Hence, we always take $\xi_{*}$ to be the right singular vector corresponding to the smallest singular value, i.e.

$$
\xi_{*}=\underset{\xi \in \mathbb{R}^{2 N},|\xi|=1}{\arg \min }|(W-U) \xi| .
$$

\section{B A formal overview of the theory of shape optimization}

In this section, we give a formal overview of the theory of shape optimization, which is the technique used in Section 2.2 to search for a better shape of post. We use the notations in Section 2.1 .

Let $V(t, x)$ be a two-dimensional vector field defined for $(t, x) \in[0, \varepsilon) \times \mathbb{R}^{2}$ for some $\varepsilon>0$; it is assumed that $V(t, x)$ is smooth in all coordinates and is compactly supported in space in a neighborhood of $\gamma$ in the interior of $Y$. Then $\tilde{V}(t, x, \theta)^{T}=\left(V(t, x)^{T}, 0\right)$ is its natural threedimensional extension, defined for $(t, x, \theta) \in[0, \varepsilon) \times \mathbb{R}^{2} \times \mathbb{R}$. $V$ and $\tilde{V}$ are referred to be perturbation vector fields, under which the domain is going to be changed. Let $T_{t}$ be the transformation associated with $V(t, x)$. To be more precise, for $\forall x \in \mathbb{R}^{2}$, we solve

$$
\frac{d T(t, x)}{d t}=V(t, T(t, x)), \quad T(0, x)=x,
$$

and define $T_{t}: x \mapsto T(t, x)$. With abuse of the notation $\varepsilon$, it is known that $\left\{T_{t}\right\}_{t \in[0, \varepsilon)}$ is a family of smooth diffeomorphisms of $\mathbb{R}^{2}$ for some $\varepsilon>0$ [30, §2.9]. In particular, for $t \in[0, \varepsilon), T_{t}$ is a one-to-one transformation from $Y$ to itself with its inverse well-defined and smooth, which is called a flow map. For an arbitrary domain $\omega \subset \mathbb{R}^{2}$ with smooth boundary, let $\omega_{t}$ be the image of $\omega$ under $T_{t}$. By assumption, $\omega_{0}=\omega$ and $\omega_{t} \subset Y$. Also let $\gamma_{t}=T_{t}(\gamma)$. Let $\tilde{T}_{t}$ is the flow map in 3-D associated with $\tilde{V}$. It is easy to show that $\Omega_{t} \triangleq \omega_{t} \times[0,1]=\tilde{T}_{t}(\Omega)$. Similarly, define $\Gamma_{t}=\tilde{T}_{t}(\Gamma)$. In what follows, we shall see how integral functionals depending on $\Omega_{t}$ and/or $\Gamma_{t}$ vary as $t$ changes.

Suppose $\left\{u\left(\Omega_{t}\right)\right\}_{t \in[0, \varepsilon)}$ is a family of functions defined on $\Omega_{t}$ and determined by $\Omega_{t}$. The limit

$$
\dot{u}(\Omega, \tilde{V})(x, \theta) \triangleq \lim _{t \rightarrow 0^{+}} \frac{u\left(\Omega_{t}\right)\left(\tilde{T}_{t}(x, \theta)\right)-u(\Omega)(x, \theta)}{t}, \quad(x, \theta) \in \Omega,
$$

if well-defined, is called the material derivative of $u$ in $\Omega$ with respect to $\tilde{V}$ [30, §2.25]. The shape derivative of $u$ in $\Omega$ with respect to $\tilde{V}$ is defined to be [30, $\$ 2.30$ ]

$$
u^{\prime}(\Omega, \tilde{V})(x, \theta) \triangleq \dot{u}(\Omega, \tilde{V})(x, \theta)-\nabla(u(\Omega))(x, \theta) \cdot \tilde{V}(0, x, \theta) .
$$


In other words, the material derivative characterizes the rate of change of $u\left(\Omega_{t}\right)$ on a particle drifted by $\tilde{V}$, while the shape derivative is the rate of change of $u\left(\Omega_{t}\right)$ by canceling the convective effect of $\tilde{T}_{t}$. In the sequel, we shall always write material and shape derivatives of $u$ as $\dot{u}$ and $u^{\prime}$ respectively. The usual derivatives will be denoted in a more explicit way, e.g. $\frac{d}{d t}$.

Let $\left\{z\left(\Gamma_{t}\right)\right\}_{t \in[0, \varepsilon)}$ be a family of functions defined on $\Gamma_{t}$ and determined by $\Gamma_{t}$. Note that $\Gamma$ is a submanifold in $\mathbb{R}^{3}$ with co-dimension 1 . The material derivative of $z$ with respect to $\tilde{V}$ is defined to be

$$
\dot{z}(\Gamma, \tilde{V})(x, \theta) \triangleq \lim _{t \rightarrow 0^{+}} \frac{z\left(\Gamma_{t}\right)\left(\tilde{T}_{t}(x, \theta)\right)-z(\Gamma)(x, \theta)}{t}, \quad(x, \theta) \in \Gamma,
$$

and the shape derivative is defined to be

$$
z^{\prime}(\Gamma, \tilde{V})(x, \theta) \triangleq \dot{z}(\Gamma, \tilde{V})(x, \theta)-\nabla_{\Gamma} z(\Gamma)(x, \theta) \cdot \tilde{V}(0, x, \theta)
$$

Here [30, §2.19]

$$
\nabla_{\Gamma} z=\left.\nabla \tilde{z}\right|_{\Gamma}-\frac{\partial \tilde{z}}{\partial n_{\Gamma}} n_{\Gamma}
$$

where $\tilde{z}$ is an arbitrary extension of $z$ from $\Gamma$ to its ambient space, and $n_{\Gamma}$ is the outer unit normal vector on $\Gamma$ with respect to $\Omega$ defined as before. For conciseness, in what follows, we shall always denote both $\tilde{V}(0, x, \theta)$ and its trace on $\Gamma$ by $\tilde{V}(0)$.

Remark B.1. For any family of functions $u\left(\Omega_{t}\right)$ defined in $\Omega_{t}$ and determined by $\Omega_{t}$, if its material and shape derivatives are well-defined, then $\left(\left.u\right|_{\Gamma}\right)^{\cdot}=\left.\dot{u}\right|_{\Gamma}$. Here $\left(\left.u\right|_{\Gamma}\right)^{*}$ is the material derivative of $\left\{\left.u\left(\Omega_{t}\right)\right|_{\Gamma_{t}}\right\}_{t \in[0, \varepsilon)}$, treated as a family of functions defined on $\Gamma_{t}$ and determined by $\Gamma_{t}$, while $\left.\dot{u}\right|_{\Gamma}$ is the restriction of $\dot{u}$ on $\Gamma$. However,

$$
\left(\left.u\right|_{\Gamma}\right)^{\prime}=\left.u^{\prime}\right|_{\Gamma}+\frac{\partial u}{\partial n_{\Gamma}}\left(\tilde{V}(0) \cdot n_{\Gamma}\right) .
$$

Here $\left(\left.u\right|_{\Gamma}\right)^{\prime}$ is the shape derivative of $\left\{\left.u\left(\Omega_{t}\right)\right|_{\Gamma_{t}}\right\}_{t \in[0, \varepsilon)}$ as a family of functions defined on $\Gamma_{t}$ and determined by $\Gamma_{t}$, while $\left.u^{\prime}\right|_{\Gamma}$ is the restriction of $u^{\prime}$ on $\Gamma$, also written as $u^{\prime}$ for simplicity. We shall always use these notations to distinguish the two different quantities.

The following two formulae are useful in calculating Eularian (or directional) derivatives of integral functionals with respect to $\tilde{V}$.

1. Suppose $u\left(\Omega_{t}\right)$ is a function on $\Omega_{t}$ and determined by $\Omega_{t}$. Then the derivative of the domain integral

$$
J_{1}\left(\Omega_{t}\right)=\int_{\Omega_{t}} u\left(\Omega_{t}\right) d x d \theta
$$

with respect to the vector field $\tilde{V}$ is that $[30,(2.168)]$

$$
\left.d J_{1}(\Omega ; \tilde{V}) \triangleq \frac{d J_{1}\left(\Omega_{t}\right)}{d t}\right|_{t=0}=\int_{\Omega} u^{\prime}(\Omega ; \tilde{V}) d x d \theta+\int_{\Gamma} u(\Omega)\left(\tilde{V}(0) \cdot n_{\Gamma}\right) d \Gamma .
$$

Here we used the fact that $\tilde{V}(t, \cdot, \theta)$ is supported in the interior of $Y$ and has zero $\theta$-component everywhere. Note that only $\Gamma$ comes into the second integral but no other parts of $\partial \Omega$.

2. Suppose $z=z\left(\Gamma_{t}\right)$ is a function defined on $\Gamma_{t}$ and determined by $\Gamma_{t}$. Then the derivative of the boundary integral

$$
J_{2}\left(\Gamma_{t}\right)=\int_{\Gamma_{t}} z\left(\Gamma_{t}\right) d \Gamma_{t}
$$

with respect to the vector field $\tilde{V}$ is that [30, (2.173) and (2.174)]

$$
\left.d J_{2}(\Gamma ; \tilde{V}) \triangleq \frac{d J_{2}\left(\Gamma_{t}\right)}{d t}\right|_{t=0}=\int_{\Gamma} z^{\prime}(\Gamma ; \tilde{V})-\kappa z(\Gamma)\left(\tilde{V}(0) \cdot n_{\Gamma}\right) d \Gamma
$$


where $\kappa$ is the sum of principal curvatures of $\Gamma$, or equivalently, the curvature of $\gamma$ in our context. In particular, if $z\left(\Gamma_{t}\right)=\left.u\left(\Omega_{t}\right)\right|_{\Gamma_{t}}$, where $u\left(\Omega_{t}\right)$ is some function defined on $\Omega_{t}$ and determined by $\Omega_{t}$,

$$
z^{\prime}(\Gamma ; \tilde{V})=\left.u^{\prime}(\Omega ; \tilde{V})\right|_{\Gamma}+\frac{\partial u}{\partial n_{\Gamma}}(\Omega)\left(\tilde{V}(0) \cdot n_{\Gamma}\right) .
$$

Hence,

$$
d J_{2}(\Omega ; \tilde{V})=\left.\int_{\Gamma} u^{\prime}(\Omega ; \tilde{V})\right|_{\Gamma}+\left(\frac{\partial u}{\partial n_{\Gamma}}(\Omega)-\kappa u(\Gamma)\right) \tilde{V}(0) \cdot n_{\Gamma} d \Gamma
$$

The following theorem states that the Eulerian derivative with respect to vector field $\tilde{V}$ of a functional in a smooth domain $\Omega$ can always have an integral representation involving only the normal component of $\tilde{V}(0)$ on $\partial \Omega$.

Theorem B.1 (The Hadamard Formula [30, Theorem 2.27]). Let $J$ be a real-valued shape functional defined on a smooth domain $\Omega$, which is shape differentiable. There exists a scalar distribution $g_{J}(\partial \Omega) \in\left(C^{\infty}(\partial \Omega)\right)^{\prime}$ on $\partial \Omega$, such that for $\forall \tilde{V} \in C^{\infty}([0, \varepsilon) \times \Omega)$,

$$
d J(\Omega ; \tilde{V})=\left\langle g_{J}(\partial \Omega),\left.\tilde{V}(0)\right|_{\partial \Omega} \cdot n_{\partial \Omega}\right\rangle_{\partial \Omega},
$$

where $\left.\tilde{V}(0)\right|_{\partial \Omega}$ is the trace of $\tilde{V}(0)$ on $\partial \Omega ;\langle\cdot, \cdot\rangle_{\partial \Omega}$ is the pairing of distribution and smooth functions on $\partial \Omega$; and $n_{\partial \Omega}$ is the unit outer normal vector to $\Omega$. If $g(\partial \Omega) \in L^{1}(\partial \Omega)$,

$$
d J(\Omega ; \tilde{V})=\int_{\partial \Omega} g_{J}(\partial \Omega)\left(\left.\tilde{V}(0)\right|_{\partial \Omega} \cdot n_{\partial \Omega}\right) d A .
$$

Remark B.2. By assumption, $\tilde{V}$ has zero $\theta$-component everywhere and is only supported in a neighborhood of $\Gamma$ in the interior of $Y \times \mathbb{R}$. Hence, B.5 could be rewritten as

$$
d J(\Omega ; \tilde{V})=\int_{\Gamma} g_{J}(\partial \Omega)\left(\tilde{V}(0) \cdot n_{\Gamma}\right) d \Gamma=\left.\int_{\gamma} d \gamma\left(V(0) \cdot n_{\gamma}\right) \int_{0}^{1} d \theta g_{J}(\partial \Omega)\right|_{\Gamma},
$$

where $V(0)=\left.V(0, \cdot)\right|_{\gamma}$. Here $\left.g(\partial \Omega)\right|_{\Gamma}$ is understood as a function of $(x, \theta) \in \gamma \times[0,1]$, and thus $\left.\int_{0}^{1} d \theta g_{J}(\partial \Omega)\right|_{\Gamma}$ is a function in $x \in \gamma$.

Formally, the shape functional $J$ increases fastest if $\gamma$ evolves in the direction given by $V(0)=$ $\left.n_{\gamma} \cdot \int_{0}^{1} d \theta g_{J}(\partial \Omega)\right|_{\Gamma}$. In the context of multi-variable calculus, this corresponds exactly to the steepest ascent method in maximizing a smooth multi-variable function $f$. Hence, in what follows, we shall find out the representation of $\left.\int_{0}^{1} d \theta g_{E}(\partial \Omega)\right|_{\Gamma}$, associated to the shape functional $E(\Omega)$, in terms of the shape derivatives $p^{\prime}$ and $p_{B}^{ \pm \prime}$, the given functions $r_{\text {in }}(\kappa), r_{\text {out }}(\kappa), \rho_{ \pm}(\beta)$ and $\tau_{ \pm}(\beta)$, and other auxiliary functions. By letting $\gamma$ evolve by the steepest ascent method, we can find a better shape of $\omega$ (or equivalently $\Omega=\omega \times[0,1]$ ) that induces larger $E(\Omega)$.

\section{Derivation of equations for the shape derivatives $p^{\prime}$ and $p_{B}^{ \pm \prime}$}

Let $\omega_{t}, \Omega_{t}, \gamma_{t}$ and $\Gamma_{t}$ be defined as before. Denote $\left(p^{t}, p_{B}^{+, t}, p_{B}^{-, t}\right)$ to be the normalized solution of the equations 2.7 - 2.12 with $\Omega$ and $\gamma$ replaced by $\Omega_{t}$ and $\gamma_{t}$ respectively. 


\section{C.1 Equations for $p^{\prime}$}

We start from the equation for $p^{t}$ in $\Omega^{t}$.

$$
\begin{aligned}
& \operatorname{div}\left(D \nabla p^{t}(x, \theta)\right)-v_{0}(\cos 2 \pi \theta, \sin 2 \pi \theta, 0) \cdot \nabla p^{t}(x, \theta)=0, \quad(x, \theta) \in \Omega_{t}, \\
& D_{t} \frac{\partial p^{t}}{\partial n_{\Gamma_{t}}}(x, \theta)-v_{0} p^{t}(x, \theta) \cos \beta^{t}+r_{\mathrm{in}}\left(\kappa^{t}(x)\right) p^{t}(x, \theta)\left[\rho_{+}\left(\beta^{t}\right)+\rho_{-}\left(\beta^{t}\right)\right]=g^{t}(x, \theta), \quad(x, \theta) \in \Gamma_{t}, \\
& g^{t}(x, \theta)=r_{\text {out }}\left(\kappa^{t}(x)\right)\left[2 \pi \tau_{+}\left(\beta^{t}\right) p_{B}^{+, t}(x)+2 \pi \tau_{-}\left(\beta^{t}\right) p_{B}^{-, t}(x)\right], \\
& \beta^{t}=2 \pi \theta-\alpha^{t}(x), \\
& p^{t} \text { satisfies periodic boundary condition on } \partial \Omega_{t} \backslash \Gamma_{t} .
\end{aligned}
$$

where $D=\operatorname{diag}\left(D_{t}, D_{t}, D_{r}\right)$, and $\beta^{t}=2 \pi \theta-\alpha^{t}(x) . \kappa^{t}(x)$ and $\alpha^{t}(x)$ are the curvature and the angle of the unit outer normal vector of $\gamma_{t}$, respectively.

\section{Definition C.1.}

$$
C_{\#}^{\infty}(Y \times[0,1])=\left\{\left.u\right|_{Y \times[0,1]}: u \in C^{\infty}\left(\mathbb{R}^{3}\right), u \text { is } Y \times[0,1] \text {-periodic }\right\} .
$$

Recall that $Y=\left[-\frac{a}{2}, \frac{a}{2}\right] \times\left[-\frac{b}{2}, \frac{b}{2}\right]$.

The weak formulation of C.1] is that, for $\forall \varphi \in C_{\#}^{\infty}(Y \times[0,1])$,

$$
\begin{aligned}
\int_{\Omega_{t}}\left(D \nabla p^{t}\right) \cdot \nabla \varphi & -v_{0} p^{t}(\cos 2 \pi \theta, \sin 2 \pi \theta, 0) \cdot \nabla \varphi \\
& =\int_{\Gamma_{t}} \varphi\left(g^{t}(x, \theta)-r_{\mathrm{in}}\left(\kappa^{t}(x)\right) p^{t}(x, \theta)\left[\rho_{+}\left(\beta^{t}\right)+\rho_{-}\left(\beta^{t}\right)\right]\right) .
\end{aligned}
$$

Take $t$-derivative on both sides at $t=0$. By (B.1) (see also [30, pp. 120]) and the fact that $\varphi^{\prime}=0$, we find that the Eulerian derivative of the left hand side of $(\mathrm{C} .2)$ is

$$
\begin{aligned}
& \int_{\Omega}\left(D \nabla p^{\prime}\right) \cdot \nabla \varphi-v_{0} p^{\prime}(\cos 2 \pi \theta, \sin 2 \pi \theta, 0) \cdot \nabla \varphi \\
& +\int_{\Gamma}\left[(D \nabla p) \cdot \nabla \varphi-v_{0} p(\cos 2 \pi \theta, \sin 2 \pi \theta, 0) \cdot \nabla \varphi\right]\left(\tilde{V}(0) \cdot n_{\Gamma}\right) .
\end{aligned}
$$

Recall that $\tilde{V}(t, \cdot, \theta)$ is compactly supported in the interior of $Y$ and has zero $\theta$-components everywhere. Hence the second term above only involves an integral on $\Gamma$ instead of on the entire $\partial \Omega$. Similarly, by $(\mathrm{B} .2)$ and $(\mathrm{B} .3)$, the Eulerian derivative of the right hand side of $(\mathrm{C} .2)$ is

$$
\begin{aligned}
& \int_{\Gamma}\left(\varphi g-\varphi r_{\mathrm{in}} p\left(\rho_{+}+\rho_{-}\right)\right)^{\prime}-\int_{\Gamma} \kappa\left(\varphi g-\varphi r_{\mathrm{in}} p\left(\rho_{+}+\rho_{-}\right)\right)\left(\tilde{V}(0) \cdot n_{\Gamma}\right) \\
= & \int_{\Gamma} \varphi g^{\prime}-\varphi\left(r_{\mathrm{in}}\left(\rho_{+}+\rho_{-}\right)\right)^{\prime} p-\varphi r_{\mathrm{in}}\left(\rho_{+}+\rho_{-}\right)\left[\left.p^{\prime}\right|_{\Gamma}+\frac{\partial p}{\partial n_{\Gamma}} \cdot\left(\tilde{V}(0) \cdot n_{\Gamma}\right)\right] \\
& +\int_{\Gamma}\left(\left.\varphi\right|_{\Gamma}\right)^{\prime}\left(g-r_{\mathrm{in}} p\left(\rho_{+}+\rho_{-}\right)\right)-\int_{\Gamma} \kappa \varphi\left(\tilde{V}(0) \cdot n_{\Gamma}\right)\left(g-r_{\mathrm{in}} p\left(\rho_{+}+\rho_{-}\right)\right),
\end{aligned}
$$

where

$$
\begin{aligned}
g^{\prime}(x, \theta)= & r_{\text {out }}^{\prime}(\kappa(x))\left[2 \pi \tau_{+}(\beta) p_{B}^{+}(x)+2 \pi \tau_{-}(\beta) p_{B}^{-}(x)\right] \\
& +r_{\text {out }}(\kappa(x)) \cdot 2 \pi\left[\tau_{+}(\beta) p_{B}^{+}(x)+\tau_{-}(\beta) p_{B}^{-}(x)\right]^{\prime} \\
= & \frac{d r_{\text {out }}}{d \kappa}(\kappa(x)) \kappa^{\prime}(x)\left[2 \pi \tau_{+}(\beta) p_{B}^{+}(x)+2 \pi \tau_{-}(\beta) p_{B}^{-}(x)\right] \\
& +r_{\text {out }}(\kappa(x)) \cdot 2 \pi\left(-\alpha^{\prime}(x)\right) \cdot\left[\frac{d \tau_{+}}{d \beta}(\beta) p_{B}^{+}(x)+\frac{d \tau_{-}}{d \beta}(\beta) p_{B}^{-}(x)\right] \\
& +r_{\text {out }}(\kappa(x)) \cdot 2 \pi\left[\tau_{+}(\beta) p_{B}^{+\prime}(x)+\tau_{-}(\beta) p_{B}^{-\prime}(x)\right]
\end{aligned}
$$




$$
\left[r_{\mathrm{in}}\left(\rho_{+}+\rho_{-}\right)\right]^{\prime}=\frac{d r_{\mathrm{in}}}{d \kappa}(\kappa(x)) \kappa^{\prime}(x)\left(\rho_{+}+\rho_{-}\right)+r_{\mathrm{in}}(\kappa(x))\left(-\alpha^{\prime}(x)\right) \cdot\left[\frac{d \rho_{+}}{d \beta}(\beta)+\frac{d \rho_{-}}{d \beta}(\beta)\right],
$$

and [30, pp. 118]

$$
\left(\left.\varphi\right|_{\Gamma}\right)^{\prime}(x, \theta)=\frac{\partial \varphi}{\partial n_{\Gamma}}(x, \theta)\left(\tilde{V}(0) \cdot n_{\Gamma}\right), \quad \forall \varphi \in C_{\#}^{\infty}(Y \times[0,1]) .
$$

To this end, if we take $\varphi \in C_{0, \#}^{\infty}(\Omega) \subset C_{\#}^{\infty}(Y \times[0,1])$, all the integrals on $\Gamma$ vanish in (C.3) and (C.4). By equating (C.3) and (C.4), we find that

$$
\int_{\Omega}\left(D \nabla p^{\prime}\right) \cdot \nabla \varphi-v_{0} p^{\prime}(\cos 2 \pi \theta, \sin 2 \pi \theta, 0) \cdot \nabla \varphi=0,
$$

which gives the equation for $p^{\prime}$

$$
\operatorname{div}\left(D \nabla p^{\prime}\right)=v_{0}(\cos 2 \pi \theta, \sin 2 \pi \theta, 0) \cdot \nabla p^{\prime}, \quad(x, \theta) \in \Omega .
$$

Then we alternatively take $\varphi \in C_{\#}^{\infty}(Y \times[0,1])$ in C.3 and C.4 such that $\frac{\partial \varphi}{\partial n_{\Gamma}}=0$ on $\Gamma$. This gives

$$
\begin{aligned}
\mathrm{C.3}= & \int_{\Gamma}\left[\left(D \nabla p^{\prime}\right) \cdot n_{\Gamma}\right] \varphi-\left.v_{0} p^{\prime}\right|_{\Gamma} \varphi(\cos 2 \pi \theta, \sin 2 \pi \theta, 0) \cdot n_{\Gamma} \\
& +\int_{\Gamma}\left[(D \nabla p) \cdot \nabla_{\Gamma} \varphi-v_{0} p(\cos 2 \pi \theta, \sin 2 \pi \theta, 0) \cdot \nabla_{\Gamma} \varphi\right]\left(\tilde{V}(0) \cdot n_{\Gamma}\right) .
\end{aligned}
$$

To obtain the first line in (C.9), we applied C.8 and integration by parts. Note that $\nabla \varphi=\nabla_{\Gamma} \varphi$ by the assumption $\frac{\partial \varphi}{\partial n_{\Gamma}}=0$. By integration by parts on $\Gamma$, C.9 can be rewritten as

$$
\begin{aligned}
\text { C.3 }= & \int_{\Gamma} D_{t} \frac{\partial p^{\prime}}{\partial n_{\Gamma}} \varphi-v_{0} p^{\prime} \varphi \cos \beta \\
& -\int_{\Gamma} \varphi \operatorname{div}_{\Gamma}\left[\left(\tilde{V}(0) \cdot n_{\Gamma}\right) D \nabla_{\Gamma} p-v_{0}\left(\tilde{V}(0) \cdot n_{\Gamma}\right) p \mathbb{P}_{\Gamma}(\cos 2 \pi \theta, \sin 2 \pi \theta, 0)\right]
\end{aligned}
$$

where $\mathbb{P}_{\Gamma}$ is the projection to the tangent space of $\Gamma$. Here we used the fact that $D \nabla_{\Gamma} p$ is tangent to $\Gamma$, and $(\cos 2 \pi \theta, \sin 2 \pi \theta, 0)^{T} \cdot n_{\Gamma}=\cos (2 \pi \theta-\alpha(x))=\cos \beta$.

Combining C.4 , C.5 , C.6), C.7) and (C.10), we obtain the boundary condition for $p^{\prime}$

$$
\begin{aligned}
D_{t} \frac{\partial p^{\prime}}{\partial n_{\Gamma}}- & v_{0} p^{\prime} \cos \beta+r_{\text {in }}\left(\rho_{+}+\rho_{-}\right) p^{\prime} \\
= & r_{\text {out }}(\kappa(x)) \cdot 2 \pi\left[\tau_{+}(\beta) p_{B}^{+\prime}(x)+\tau_{-}(\beta) p_{B}^{-\prime}(x)\right] \\
& +\frac{d r_{\text {out }}}{d \kappa}(\kappa(x)) \kappa^{\prime}(x)\left[2 \pi \tau_{+}(\beta) p_{B}^{+}(x)+2 \pi \tau_{-}(\beta) p_{B}^{-}(x)\right] \\
& +r_{\text {out }}(\kappa(x)) \cdot 2 \pi\left(-\alpha^{\prime}(x)\right) \cdot\left[\frac{d \tau_{+}}{d \beta}(\beta) p_{B}^{+}(x)+\frac{d \tau_{-}}{d \beta}(\beta) p_{B}^{-}(x)\right] \\
& -\frac{d r_{\text {in }}}{d \kappa}(\kappa(x)) \kappa^{\prime}(x)\left(\rho_{+}(\beta)+\rho_{-}(\beta)\right) p \\
& -r_{\text {in }}(\kappa(x))\left(-\alpha^{\prime}(x)\right) \cdot\left[\frac{d \rho_{+}}{d \beta}(\beta)+\frac{d \rho_{-}}{d \beta}(\beta)\right] p \\
& -r_{\text {in }}\left(\rho_{+}+\rho_{-}\right) \frac{\partial p}{\partial n_{\Gamma}}\left(\tilde{V}(0) \cdot n_{\Gamma}\right)-\kappa\left(\tilde{V}(0) \cdot n_{\Gamma}\right)\left(g-r_{\text {in }} p\left(\rho_{+}+\rho_{-}\right)\right) \\
& +\operatorname{div}_{\Gamma}\left[\left(\tilde{V}(0) \cdot n_{\Gamma}\right) D \nabla_{\Gamma} p-v_{0}\left(\tilde{V}(0) \cdot n_{\Gamma}\right) p \mathbb{P}_{\Gamma}(\cos 2 \pi \theta, \sin 2 \pi \theta, 0)\right],
\end{aligned}
$$

where $g$ is given by A.1. Here

$$
\begin{aligned}
\alpha^{\prime}(s) & =\partial_{s}[V(0) \cdot n(s)], \\
\kappa^{\prime}(s) & =\partial_{s s}(V(0) \cdot n(s))+\kappa^{2}(s)(V(0) \cdot n(s)),
\end{aligned}
$$

which will be shown in Section C.3. 


\section{C.2 Equations for $p_{B}^{ \pm \prime}$}

Let $s_{t}$ be the arclength parameter of $\gamma_{t}$. The equations for $p_{B}^{ \pm, t}$ on $\gamma_{t}$ are

$$
-D_{t} \Delta_{\gamma_{t}} p_{B}^{ \pm, t}(x) \pm v_{0} \partial_{s_{t}} p_{b}^{ \pm, t}(x)+r_{\text {out }}\left(\kappa^{t}(x)\right) p_{B}^{ \pm, t}(x)=r_{\text {in }}\left(\kappa^{t}(x)\right) \int_{0}^{1} \rho_{ \pm}\left(\beta^{t}\right) p^{t}(x, \theta) d \theta .
$$

Their weak formulations are that, for $\forall \psi \in \mathcal{D}_{\#}(Y)$,

$$
\int_{\gamma_{t}}-\left.\left.D_{t} p_{B}^{ \pm, t} \Delta_{\gamma_{t}} \psi\right|_{\gamma_{t}} \mp v_{0} \partial_{s_{t}} \psi\right|_{\gamma_{t}} p_{B}^{ \pm, t}+\left.r_{\text {out }}\left(\kappa^{t}\right) p_{B}^{ \pm, t} \psi\right|_{\gamma_{t}}=\left.\int_{\gamma_{t}} \psi\right|_{\gamma_{t}} r_{\text {in }}\left(\kappa^{t}\right) \int_{0}^{1} d \theta \rho_{ \pm}\left(\beta^{t}\right) p^{t}(x, \theta) .
$$

Take $t$-derivative at $t=0$ on both sides of (C.14). By (B.4), we find the Eulerian derivative of the right hand side of $(\mathrm{C.14})$ is

$$
\begin{aligned}
& \int_{\Gamma}\left[\left.\psi\right|_{\gamma} r_{\mathrm{in}}(\kappa(x)) \rho_{ \pm}(\beta) p(x, \theta)\right]^{\prime}-\int_{\Gamma} \kappa(V(0) \cdot n) \psi r_{\mathrm{in}}(\kappa(x)) \rho_{ \pm}(\beta) p(x, \theta) \\
= & \int_{\Gamma} \frac{\partial \psi}{\partial n}(V(0) \cdot n) r_{\mathrm{in}}(\kappa(x)) \rho_{ \pm}(\beta) p(x, \theta)+\int_{\Gamma} \psi \frac{d r_{\mathrm{in}}}{d \kappa}(\kappa(x)) \kappa^{\prime}(x) \rho_{ \pm}(\beta) p(x, \theta) \\
& +\int_{\Gamma} \psi r_{\mathrm{in}}(\kappa(x)) \frac{d \rho_{ \pm}}{d \beta}(\beta)\left(-\alpha^{\prime}(x)\right) p(x, \theta) \\
& +\int_{\Gamma} \psi r_{\mathrm{in}}(\kappa(x)) \rho_{ \pm}\left[\left.p^{\prime}(x, \theta)\right|_{\Gamma}+\frac{\partial p}{\partial n_{\Gamma}}(x, \theta)(V(0) \cdot n)\right]-\int_{\Gamma} \kappa(V(0) \cdot n) \psi r_{\mathrm{in}} \rho_{ \pm} p(x, \theta) .
\end{aligned}
$$

Here the functions originally defined for $x \in \gamma$ are interpreted in a natural way as functions defined for $(x, \theta) \in \Gamma=\gamma \times[0,1]$. On the other hand, the Eulerian derivative of the left hand side of C.14 is that

$$
\begin{aligned}
& \int_{\gamma}\left[-\left.\left.D_{t} p_{B}^{ \pm} \Delta_{\gamma} \psi\right|_{\gamma} \mp v_{0} \partial_{s} \psi\right|_{\gamma} p_{B}^{ \pm}+\left.r_{\text {out }}(\kappa(x)) p_{B}^{ \pm} \psi\right|_{\gamma}\right]^{\prime} \\
& -\int_{\gamma} \kappa(V(0) \cdot n)\left(-D_{t} p_{B}^{ \pm} \Delta_{\gamma} \psi \mp v_{0} \partial_{s} \psi p_{B}^{ \pm}+r_{\text {out }}(\kappa(x)) p_{B}^{ \pm} \psi\right) \\
= & \int_{\gamma}-D_{t} p_{B}^{ \pm \prime} \Delta_{\gamma} \psi \mp v_{0} \partial_{s} \psi p_{B}^{ \pm \prime}+r_{\text {out }}(\kappa(x)) p_{B}^{ \pm \prime} \psi+\frac{d r_{\text {out }}}{d \kappa}(\kappa(x)) \kappa^{\prime}(x) p_{B}^{ \pm} \psi \\
& +\int_{\gamma}-D_{t} p_{B}^{ \pm}\left(\left.\Delta_{\gamma} \psi\right|_{\gamma}\right)^{\prime} \mp v_{0} p_{B}^{ \pm}\left(\left.\partial_{s} \psi\right|_{\gamma}\right)^{\prime}+r_{\text {out }}(\kappa(x)) p_{B}^{ \pm} \frac{\partial \psi}{\partial n}(V(0) \cdot n) \\
& -\int_{\gamma} \kappa(V(0) \cdot n)\left(-D_{t} p_{B}^{ \pm} \Delta_{\gamma} \psi \mp v_{0} \partial_{s} \psi p_{B}^{ \pm}+r_{\text {out }}(\kappa(x)) p_{B}^{ \pm} \psi\right) .
\end{aligned}
$$

To further simplify (C.16), we need the formulae for $\left(\left.\Delta_{\gamma} \psi\right|_{\gamma}\right)^{\prime}$ and $\left(\left.\partial_{s} \psi\right|_{\gamma}\right)^{\prime}$ derived in Section C.4.

$$
\begin{aligned}
\left(\partial_{s} \psi\right)^{\prime} & =\kappa(V(0) \cdot n) \partial_{s} \psi+\partial_{s}\left(\frac{\partial \psi}{\partial n}(V(0) \cdot n)\right) \\
\left(\left.\Delta_{\gamma} \psi\right|_{\gamma}\right)^{\prime} & =\partial_{s}[\kappa(V(0) \cdot n)] \cdot \partial_{s} \psi+\partial_{s s}\left(\frac{\partial \psi}{\partial n}(V(0) \cdot n)\right)+2 \kappa(V(0) \cdot n) \partial_{s s} \psi .
\end{aligned}
$$

Hence, C.16 becomes

$$
\begin{aligned}
\text { C.16 }= & \int_{\gamma}-D_{t} p_{B}^{ \pm \prime} \Delta_{\gamma} \psi \mp v_{0} \partial_{s} \psi p_{B}^{ \pm \prime}+r_{\text {out }}(\kappa(x)) p_{B}^{ \pm \prime} \psi+\frac{d r_{\text {out }}}{d \kappa}(\kappa(x)) \kappa^{\prime}(x) p_{B}^{ \pm} \psi \\
& +\int_{\gamma}-D_{t} p_{B}^{ \pm}\left(\partial_{s}[\kappa(V(0) \cdot n)] \cdot \partial_{s} \psi+\partial_{s s}\left(\frac{\partial \psi}{\partial n}(V(0) \cdot n)\right)+2 \kappa(V(0) \cdot n) \partial_{s s} \psi\right) \\
& \mp \int_{\gamma} v_{0} p_{B}^{ \pm}\left(\kappa(V(0) \cdot n) \partial_{s} \psi+\partial_{s}\left(\frac{\partial \psi}{\partial n}(V(0) \cdot n)\right)\right)+\int_{\gamma} r_{\text {out }}(\kappa(x)) p_{B}^{ \pm} \frac{\partial \psi}{\partial n}(V(0) \cdot n) \\
& -\int_{\gamma} \kappa(V(0) \cdot n)\left(-D_{t} p_{B}^{ \pm} \Delta_{\gamma} \psi \mp v_{0} \partial_{s} \psi p_{B}^{ \pm}+r_{\text {out }}(\kappa(x)) p_{B}^{ \pm} \psi\right) .
\end{aligned}
$$


We take $\psi$ such that $\frac{\partial \psi}{\partial n}=0$ on $\gamma$ and do integration by parts

$$
\begin{aligned}
\text { C.16 }= & \int_{\gamma}-D_{t} \Delta_{\gamma} p_{B}^{ \pm \prime} \psi \pm v_{0} \psi \partial_{s} p_{B}^{ \pm \prime}+r_{\text {out }}(\kappa(x)) p_{B}^{ \pm \prime} \psi+\frac{d r_{\text {out }}}{d \kappa}(\kappa(x)) \kappa^{\prime}(x) p_{B}^{ \pm} \psi \\
& -\int_{\gamma} D_{t} \partial_{s}\left(\partial_{s}[\kappa(V(0) \cdot n)] p_{B}^{ \pm}\right) \cdot \psi-2 D_{t} \int_{\gamma} \partial_{s}\left[\kappa(V(0) \cdot n) \partial_{s} p_{B}^{ \pm}\right] \cdot \psi \\
& \pm \int_{\gamma} v_{0} \partial_{s}\left[p_{B}^{ \pm} \kappa(V(0) \cdot n)\right] \psi \\
& -\int_{\gamma}-D_{t} \Delta_{\gamma}\left[\kappa(V(0) \cdot n) p_{B}^{ \pm}\right] \psi \pm v_{0} \psi \partial_{s}\left[\kappa(V(0) \cdot n) p_{B}^{ \pm}\right]+r_{\text {out }}(\kappa(x)) \kappa(V(0) \cdot n) p_{B}^{ \pm} \psi \\
= & \int_{\gamma}-D_{t} \Delta_{\gamma} p_{B}^{ \pm \prime} \psi \pm v_{0} \psi \partial_{s} p_{B}^{ \pm \prime}+r_{\text {out }}(\kappa(x)) p_{B}^{ \pm \prime} \psi+\frac{d r_{\text {out }}}{d \kappa}(\kappa(x)) \kappa^{\prime}(x) p_{B}^{ \pm} \psi \\
& -D_{t} \int_{\gamma} \partial_{s}\left[\kappa(V(0) \cdot n) \partial_{s} p_{B}^{ \pm}\right] \cdot \psi-\int_{\gamma} r_{\text {out }}(\kappa(x)) \kappa(V(0) \cdot n) p_{B}^{ \pm} \psi .
\end{aligned}
$$

On the other hand, with $\frac{\partial \psi}{\partial n}=0$, C.15 becomes

$$
\begin{aligned}
\text { C.15 }= & \int_{\Gamma} \psi \frac{d r_{\mathrm{in}}}{d \kappa}(\kappa(x)) \kappa^{\prime}(x) \rho_{ \pm}(\beta) p(x, \theta)+\int_{\Gamma} \psi r_{\mathrm{in}}(\kappa(x)) \frac{d \rho_{ \pm}}{d \beta}(\beta)\left(-\alpha^{\prime}(x)\right) p(x, \theta) \\
& +\int_{\Gamma} \psi r_{\mathrm{in}}(\kappa(x)) \rho_{ \pm}\left[\left.p^{\prime}(x, \theta)\right|_{\Gamma}+\frac{\partial p}{\partial n_{\Gamma}}(x, \theta)(V(0) \cdot n)\right]-\int_{\Gamma} \kappa(V(0) \cdot n) \psi r_{\mathrm{in}} \rho_{ \pm} p(x, \theta) .
\end{aligned}
$$

Equating C.17) and (C.18), we immediately find the equations for $p_{B}^{ \pm \prime}$

$$
\begin{aligned}
& -D_{t} \Delta_{\gamma} p_{B}^{ \pm \prime} \pm v_{0} \partial_{s} p_{B}^{ \pm \prime}+r_{\text {out }}(\kappa(x)) p_{B}^{ \pm \prime} \\
= & \int_{0}^{1} \frac{d r_{\text {in }}}{d \kappa}(\kappa(x)) \kappa^{\prime}(x) \rho_{ \pm} p(x, \theta)+r_{\text {in }}(\kappa(x)) \frac{d \rho_{ \pm}}{d \beta}(\beta)\left(-\alpha^{\prime}(x)\right) p(x, \theta) d \theta \\
& +\int_{0}^{1} r_{\text {in }}(\kappa(x)) \rho_{ \pm}\left[\left.p^{\prime}(x, \theta)\right|_{\Gamma}+\frac{\partial p}{\partial n_{\Gamma}}(x, \theta)(V(0) \cdot n)\right]-\kappa(V(0) \cdot n) r_{\text {in }} \rho_{ \pm} p(x, \theta) d \theta \\
& -\frac{d r_{\text {out }}}{d \kappa}(\kappa(x)) \kappa^{\prime}(x) p_{B}^{ \pm}+D_{t} \partial_{s}\left[\kappa(V(0) \cdot n) \partial_{s} p_{B}^{ \pm}\right]+r_{\text {out }}(\kappa(x)) \kappa(V(0) \cdot n) p_{B}^{ \pm} .
\end{aligned}
$$

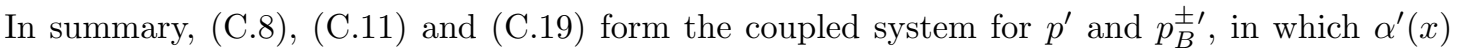
and $\kappa^{\prime}(x)$ are given by (C.24) and C.26 respectively. As before, $\beta=2 \pi \theta-\alpha(x)$ is defined for $(x, \theta) \in \Gamma$.

\section{C.3 Derivation of $\kappa^{\prime}(x)$ and $\alpha^{\prime}(x)$}

In this section, we shall derive explicit formulae for $\kappa^{\prime}(x)$ and $\alpha^{\prime}(x)$, which appear in the equations of $p^{\prime}$ and $p_{B}^{ \pm \prime}$. For the elementary differential geometry involved in the following calculation, readers are referred to 38 .

Let $s$ denote the arclength parameter of $\gamma$ at $t=0$. Let $\tau(s)=\partial_{s} \gamma(s)$ denote the unit tangent vector of $\gamma$ at $t=0$, and let $n(s)$ be the unit normal vector pointing towards $O$, the domain enclosed by $\gamma$. We shall write $V(0, \gamma(s))=V(0)$ whenever it is convenient.

By definition, the unit tangent vector of $\gamma_{t}$ at time $t$ is given by

$$
\tau^{t}(s)=\frac{\frac{d}{d s}\left(T_{t} \circ \gamma\right)(s)}{\left|\frac{d}{d s}\left(T_{t} \circ \gamma\right)(s)\right|}=\frac{\nabla T_{t}(\gamma(s)) \partial_{s} \gamma(s)}{\left|\nabla T_{t}(\gamma(s)) \partial_{s} \gamma(s)\right|} .
$$


To derive the material derivative of the unit tangent vector, we take $t$-derivative at $t=0$ and find

$$
\begin{aligned}
\dot{\tau}(s) & =\frac{\nabla V(0) \partial_{s} \gamma(s)\left|\partial_{s} \gamma(s)\right|-\frac{\partial_{s} \gamma(s)}{\left|\partial_{s} \gamma(s)\right|} \partial_{s} \gamma(s)^{T} \nabla V(0) \partial_{s} \gamma(s)}{\left|\partial_{s} \gamma(s)\right|^{2}} \\
& =\nabla V(0) \partial_{s} \gamma(s)-\left[\partial_{s} \gamma(s)^{T} \nabla V(0) \partial_{s} \gamma(s)\right] \partial_{s} \gamma(s) \\
& =\left[n(s)^{T} \nabla_{\gamma} V(0) \tau(s)\right] n(s) \\
& =\left[n(s) \cdot \partial_{s} V(0)\right] n(s) .
\end{aligned}
$$

Here we used the fact that

$$
\left.\nabla T_{t}\right|_{t=0}=I d,\left.\quad \frac{d \nabla T_{t}(\gamma(s))}{d t}\right|_{t=0}=\nabla V(0)
$$

and the following orthogonal decomposition in dimension two

$$
\nabla V(0) \partial_{s} \gamma(s)=\left[\partial_{s} \gamma(s)^{T} \nabla V(0) \partial_{s} \gamma(s)\right] \partial_{s} \gamma(s)+\left[n(s)^{T} \nabla V(0) \tau(s)\right] n(s) .
$$

By definition,

$$
\tau^{\prime}(s)=\dot{\tau}(s)-V(0) \cdot \nabla_{\Gamma} \tau=\dot{\tau}(s)-[V(0) \cdot \tau(s)] \partial_{s} \tau=\dot{\tau}(s)-[V(0) \cdot \tau(s)] \kappa(s) n(s),
$$

where $\kappa(s)$ is the curvature of $\gamma$.

$\dot{n}(s)$ can be calculated easily with the observation

$$
n^{t}(s)=\left(n_{1}^{t}(s), n_{2}^{t}(s)\right)^{T}=\left(-\tau_{2}^{t}(s), \tau_{1}^{t}(s)\right)^{T} .
$$

Combining this with C.21, we find

$$
\dot{n}(s)=-\left(n(s) \cdot \partial_{s} V(0)\right) \tau(s),
$$

and thus

$$
n^{\prime}(s)=\dot{n}(s)-V(0) \cdot \nabla_{\gamma} n(s)=\dot{n}(s)-[V(0) \cdot \tau(s)] \partial_{s} n(s)=\dot{n}(s)+\kappa(s) \tau(s)[V(0) \cdot \tau(s)] .
$$

Recall that the angle $\alpha$ of the outer normal vector of $\gamma$ is determined by

$$
(\cos \alpha(s), \sin \alpha(s))^{T}=n(s) .
$$

Take material derivatives on both sides and we have

$$
(-\sin \alpha(s) \cdot \dot{\alpha}(s), \cos \alpha(s) \cdot \dot{\alpha}(s))^{T}=\dot{n}(s) .
$$

Take inner product with $-\tau(s)=(-\sin \alpha(s), \cos \alpha(s))^{T}$ and we find by C.23

$$
\dot{\alpha}(s)=-\dot{n}(s) \cdot \tau(s)=n(s) \cdot \partial_{s} V(0) .
$$

By taking derivative with respect to $s$ in the definition of $\alpha(s)$,

$$
\partial_{s} \alpha(s)(-\sin \alpha(s), \cos \alpha(s))^{T}=\partial_{s} n(s)=-\kappa(s) \tau(s) .
$$

Taking inner product with $-\tau(s)$, we obtain $\partial_{s} \alpha(s)=\kappa(s)$. Hence,

$$
\begin{aligned}
\alpha^{\prime}(s) & =\dot{\alpha}(s)-V(0) \cdot \nabla_{\gamma} \alpha=n(s) \cdot \partial_{s} V(0)-[V(0) \cdot \tau(s)] \partial_{s} \alpha(s) \\
& =\partial_{s} V(0) \cdot n(s)-\kappa(s) V(0) \cdot \tau(s)=\partial_{s}[V(0) \cdot n(s)] .
\end{aligned}
$$


Next we are going to calculate $\kappa^{\prime}(s)$. At time $t$,

$$
\kappa^{t}(s)=n^{t}(s) \cdot \frac{\frac{d}{d s} \tau^{t}(s)}{\left|\partial_{s} \gamma_{t}(s)\right|}
$$

Note that $s$ is not necessarily the arclength parameter at time $t$. By using (C.20) and

$$
\left|\partial_{s} \gamma_{t}(s)\right|=\left|\nabla T_{t}(\gamma(s)) \partial_{s} \gamma(s)\right|
$$

we know that

$$
\begin{aligned}
\kappa^{t}(s)= & \frac{1}{\left|\left(\nabla T_{t}\right) \partial_{s} \gamma(s)\right|^{4}} \cdot n_{i}^{t}(s)\left[\partial_{k}\left(\nabla T_{t}\right)_{i j} \partial_{s} \gamma_{k}(s) \partial_{s} \gamma_{j}(s)\left|\left(\nabla T_{t}\right) \partial_{s} \gamma(s)\right|^{2}\right. \\
& +\left(\nabla T_{t}\right)_{i j} \partial_{s s} \gamma_{j}(s)\left|\left(\nabla T_{t}\right) \partial_{s} \gamma(s)\right|^{2} \\
& \left.-\left(\nabla T_{t}\right)_{i j} \partial_{s} \gamma_{j}(s) \cdot \partial_{s} \gamma(s)^{T} \nabla T_{t}^{T}\left(\nabla T_{t} \partial_{s s} \gamma(s)+\partial_{k}\left(\nabla T_{t}\right) \cdot \partial_{s} \gamma_{k}(s) \partial_{s} \gamma(s)\right)\right] .
\end{aligned}
$$

Here we used Einstein summation convention and wrote $\nabla T_{t}(\gamma(s))$ as $\nabla T_{t}$. Thanks to (C.20), the last term in C.25 should vanish since

$$
n_{i}^{t}(s)\left(\nabla T_{t}\right)_{i j} \partial_{s} \gamma_{j}(s)=n^{t}(s) \cdot\left(\nabla T_{t} \tau(s)\right)=n^{t}(s) \cdot \tau^{t}(s)\left|\nabla T_{t} \tau(s)\right|=0 .
$$

This gives

$$
\kappa^{t}(s)=\frac{1}{\left|\nabla T_{t} \partial_{s} \gamma(s)\right|^{2}} \cdot n_{i}^{t}(s)\left[\partial_{k}\left(\nabla T_{t}\right)_{i j} \partial_{s} \gamma_{k}(s) \partial_{s} \gamma_{j}(s)+\left(\nabla T_{t}\right)_{i j} \partial_{s s} \gamma_{j}(s)\right]
$$

Taking $t$-derivative on both sides at $t=0$, we find by C.22 that

$$
\begin{aligned}
\dot{\kappa}(s)= & n(s)^{T}\left[\partial_{s}(\nabla V(0)) \tau(s)\right]+\dot{n}(s) \cdot \partial_{s s} \gamma(s)+n(s)^{T} \nabla V(0) \partial_{s s} \gamma(s) \\
& -\left(n(s) \cdot \partial_{s s} \gamma(s)\right) \cdot 2 \tau(s)^{T} \nabla V(0) \tau(s) \\
= & n(s)^{T} \partial_{s}(\nabla V(0)) \tau(s)-\left[\left(n(s) \cdot \partial_{s} V(0)\right) \tau(s) \cdot \kappa(s) n(s)\right]+\kappa(s) n(s)^{T} \nabla V(0) n(s) \\
& -2 \kappa(s) \tau(s)^{T} \nabla V(0) \tau(s) \\
= & n(s)^{T} \partial_{s}(\nabla V(0)) \tau(s)+\kappa(s)\left[n(s)^{T} \nabla V(0) n(s)-2 \tau(s)^{T} \nabla V(0) \tau(s)\right] .
\end{aligned}
$$

By definition,

$$
\kappa^{\prime}(s)=\dot{\kappa}(s)-\nabla_{\gamma} \kappa(s) \cdot V(0) .
$$

It is not difficult to verify that

$$
\kappa^{\prime}(s)=\partial_{s s}(V(0) \cdot n(s))+\kappa^{2}(s)(V(0) \cdot n(s)) .
$$

Indeed,

$$
\partial_{s}(V(0) \cdot n(s))=\partial_{s} V(0) \cdot n(s)-V(0) \cdot \kappa(s) \tau(s)=n(s)^{T} \nabla V(0) \tau(s)-\kappa(s)(V(0) \cdot \tau(s)),
$$

and

$$
\begin{aligned}
\partial_{s s}(V(0) \cdot n(s))= & -\kappa(s) \tau(s)^{T} \nabla V(0) \tau(s)+n(s)^{T} \partial_{s} \nabla V(0) \tau(s)+\kappa(s) n(s)^{T} \nabla V(0) n(s) \\
& -\partial_{s} \kappa(s)(V(0) \cdot \tau(s))-\kappa(s)\left(\partial_{s} V(0) \cdot \tau(s)\right)-\kappa^{2}(s)(V(0) \cdot n(s)) .
\end{aligned}
$$

Hence,

$$
\begin{aligned}
& \partial_{s s}(V(0) \cdot n(s))+\kappa^{2}(s)(V(0) \cdot n(s)) \\
= & -2 \kappa(s) \tau(s)^{T} \nabla V(0) \tau(s)+n(s)^{T} \partial_{s} \nabla V(0) \tau(s)+\kappa(s) n(s)^{T} \nabla V(0) n(s)-\partial_{s} \kappa(s)(V(0) \cdot \tau(s)) \\
= & \kappa(s)\left[n(s)^{T} \nabla V(0) n(s)-2 \tau(s)^{T} \nabla V(0) \tau(s)\right]+n(s)^{T} \partial_{s} \nabla V(0) \tau(s)-\nabla_{\gamma} \kappa(s) \cdot V(0) \\
= & \dot{\kappa}(s)-\nabla_{\gamma} \kappa(s) \cdot V(0)=\kappa^{\prime}(s) .
\end{aligned}
$$

We remark that the first term on the right hand side of C.26 shows how $V$ changes the curvature by bending $\gamma$, while the second term accounts for the effect of $V$ changing the arclength of $\gamma$. 


\section{C.4 Derivation of $\left(\left.\partial_{s} \psi\right|_{\gamma}\right)^{\prime}$ and $\left(\left.\Delta_{\gamma} \psi\right|_{\gamma}\right)^{\prime}$}

This section is devoted to calculating $\left(\left.\partial_{s} \psi\right|_{\gamma}\right)^{\prime}$ and $\left(\left.\Delta_{\gamma} \psi\right|_{\gamma}\right)^{\prime}$, where $\psi \in \mathcal{D}_{\#}(Y)$ and the derivatives are calculated under the perturbation vector field $V$ introduced in Section $\mathrm{B}$ and used before. These two quantities are used in the derivation of equations for $p_{B}^{ \pm \prime}$ in Section C.2

We start from $\left(\left.\partial_{s} \psi\right|_{\gamma}\right)$ : By (C.21),

$$
\left(\left.\partial_{s} \psi\right|_{\gamma}\right)^{\cdot}=\left(\left.\tau \cdot \partial_{\gamma} \psi\right|_{\gamma}\right)^{\cdot}=\dot{\tau} \cdot \partial_{\gamma} \psi+\tau \cdot\left(\left.\partial_{\gamma} \psi\right|_{\gamma}\right)^{\cdot}=\left(n \cdot \partial_{s} V(0)\right) n \cdot \partial_{\gamma} \psi+\tau \cdot\left(\left.\partial_{\gamma} \psi\right|_{\gamma}\right)^{*}=\tau \cdot\left(\left.\partial_{\gamma} \psi\right|_{\gamma}\right)^{\bullet}
$$

Recall that for an arbitrary smooth function $f$ defined on $\gamma, \partial_{\gamma} f=\left(\partial_{s} f\right) \tau \in \mathbb{R}^{2}$, and $\partial_{s} f=\partial_{\gamma} f \cdot \tau \in$ R. It is known that [30, (2.137) and (2.138)]

$$
\left[\left.\partial_{\gamma_{t}} \psi\right|_{\gamma_{t}}\right] \circ T_{t}=\left(\nabla T_{t}\right)^{-T} \cdot\left[\nabla\left(\psi \circ T_{t}\right)-\left((B(t) n) \cdot \nabla\left(\psi \circ T_{t}\right)\right) n\right],
$$

where $n$ is the unit normal vector of $\gamma$ defined as before and

$$
B(t)=\left|\left(\nabla T_{t}\right)^{-T} n\right|^{-2}\left(\nabla T_{t}\right)^{-1} \cdot\left(\nabla T_{t}\right)^{-T}
$$

Take $t$-derivative at $t=0$ on both sides of (C.27) and we obtain

$$
\left(\partial_{\gamma} \psi\right)^{\cdot}=\left.\frac{d}{d t}\right|_{t=0}\left(\nabla T_{t}\right)^{-T} \cdot\left[\nabla\left(\psi \circ T_{t}\right)-\left((B(t) n) \cdot \nabla\left(\psi \circ T_{t}\right)\right) n\right] .
$$

Using the fact that

$$
\begin{aligned}
\left.\left(\nabla T_{t}\right)^{-1}\right|_{t=0} & =I d,\left.\quad \frac{d}{d t}\right|_{t=0}\left(\nabla T_{t}\right)^{-1}=-\nabla V(0), \\
B(0) & =I d,\left.\quad \frac{d}{d t}\right|_{t=0} B(t)=-\nabla V(0)-\nabla V(0)^{T}+n^{T}\left(\nabla V(0)+\nabla V(0)^{T}\right) n \cdot I d, \\
\left.\nabla\left(\psi \circ T_{t}\right)\right|_{t=0} & =\nabla \psi,\left.\quad \frac{d}{d t}\right|_{t=0} \nabla\left(\psi \circ T_{t}\right)=\nabla(\nabla \psi \cdot V(0)),
\end{aligned}
$$

we find

$$
\left(\partial_{\gamma} \psi\right)^{\cdot}=-(\nabla V(0))^{T}[\nabla \psi-(n \cdot \nabla \psi) n]+[\nabla(\nabla \psi \cdot V(0))-C n]
$$

where $C=\left.\frac{d}{d t}\right|_{t=0}\left[(B(t) n) \cdot \nabla\left(\psi \circ T_{t}\right)\right]$ is a scalar. Hence,

$$
\left(\partial_{s} \psi\right)^{\cdot}=\tau \cdot\left(\partial_{\gamma} \psi\right)^{\cdot}=-\tau^{T}(\nabla V(0))^{T}[\nabla \psi-(n \cdot \nabla \psi) n]+\tau \cdot \nabla(\nabla \psi \cdot V(0)),
$$

and

$$
\begin{aligned}
\left(\partial_{s} \psi\right)^{\prime}= & \left(\partial_{s} \psi\right)^{*}-\partial_{\gamma}\left(\partial_{s} \psi\right) \cdot V(0) \\
= & -\tau^{T}(\nabla V(0))^{T}[\nabla \psi-\langle n, \nabla \psi\rangle n]+\tau \cdot \nabla(\nabla \psi \cdot V(0))-\partial_{\gamma}\left(\partial_{s} \psi\right) \cdot V(0) \\
= & -\partial_{s} V(0) \cdot \partial_{\gamma} \psi+\partial_{s}(\nabla \psi \cdot V(0))-\partial_{s s} \psi \cdot(V(0) \cdot \tau) \\
= & -\partial_{s}[(V(0) \cdot n) n+(V(0) \cdot \tau) \tau] \cdot \partial_{\gamma} \psi \\
& +\partial_{s}\left(\partial_{s} \psi(V(0) \cdot \tau)+\frac{\partial \psi}{\partial n}(V(0) \cdot n)\right)-\partial_{s s} \psi \cdot(V(0) \cdot \tau) \\
= & -\left[-\kappa(V(0) \cdot n)+\partial_{s}(V(0) \cdot \tau)\right] \partial_{s} \psi+\partial_{s} \psi \partial_{s}(V(0) \cdot \tau)+\partial_{s}\left(\frac{\partial \psi}{\partial n}(V(0) \cdot n)\right) \\
= & \kappa(V(0) \cdot n) \partial_{s} \psi+\partial_{s}\left(\frac{\partial \psi}{\partial n}(V(0) \cdot n)\right) .
\end{aligned}
$$

We remark that the first term above accounts for the change of $\partial_{s} \psi$ due to $V$ changing the arclength of $\gamma$; while the second term results from the motion of $\gamma$ by $V$ in the normal direction. 
Next we turn to $\left(\left.\Delta_{\gamma} \psi\right|_{\gamma}\right)^{\prime}$. It is known that [30, (2.147)]

$$
\left(\left.\Delta_{\gamma_{t}} \psi\right|_{\gamma_{t}}\right) \circ T_{t}=\omega(t)^{-1} \operatorname{div}_{\gamma}\left[C(t) \cdot\left(\partial_{\gamma}\left[\psi \circ T_{t}\right]-\left(n^{T} C(t) n\right)^{-1}\left[(C(t) n) \cdot \partial_{\gamma}\left(\psi \circ T_{t}\right)\right] n\right)\right],
$$

where

$$
\begin{aligned}
& \omega(t)=\operatorname{det}\left(\nabla T_{t}\right)\left|\nabla T_{t}^{-T} n\right|, \\
& C(t)=\omega(t) \nabla T_{t}^{-1} \cdot \nabla T_{t}^{-T} .
\end{aligned}
$$

To derive the material derivative $\left(\left.\Delta_{\gamma} \psi\right|_{\gamma}\right)$; we take $t$-derivative on both sides of $($ C.28 and use the following facts

$$
\begin{gathered}
\omega(0)=1,\left.\quad \frac{d}{d t}\right|_{t=0} \omega(t)=\operatorname{div} V(0)-n^{T} \nabla V(0) n=\operatorname{div}_{\gamma} V(0), \\
C(0)=I d,\left.\quad \frac{d}{d t}\right|_{t=0} C(t)=\operatorname{div}_{\gamma} V(0) \cdot I d-\nabla V(0)-\nabla V(0)^{T} .
\end{gathered}
$$

Hence,

$$
\begin{aligned}
\left(\left.\Delta_{\gamma} \psi\right|_{\gamma}\right)= & -\operatorname{div}_{\gamma} V(0) \cdot \operatorname{div}_{\gamma}\left(\partial_{\gamma} \psi\right)+\operatorname{div}_{\gamma}\left[\left(\operatorname{div}_{\gamma} V(0) \cdot I d-\nabla V(0)-\nabla V(0)^{T}\right) \partial_{\gamma} \psi\right] \\
& +\operatorname{div}_{\gamma}\left[\partial_{\gamma}(\nabla \psi \cdot V(0))-\left(\left[\operatorname{div}_{\gamma} V(0) \cdot I d-\nabla V(0)-\nabla V(0)^{T}\right] n \cdot \partial_{\gamma} \psi\right) n\right] \\
= & -\operatorname{div}_{\gamma} V(0) \cdot \Delta_{\gamma} \psi+\operatorname{div}_{\gamma}\left[\left(\operatorname{div}_{\gamma} V(0) \cdot I d-\nabla V(0)-\nabla V(0)^{T}\right) \partial_{\gamma} \psi\right] \\
& +\operatorname{div}_{\gamma}\left[\partial_{\gamma}(\nabla \psi \cdot V(0))+\left(\left[\nabla V(0)+\nabla V(0)^{T}\right] n \cdot \partial_{\gamma} \psi\right) n\right] \\
= & -\operatorname{div}_{\gamma} V(0) \cdot \Delta_{\gamma} \psi+\operatorname{div}_{\gamma}\left[\operatorname{div}_{\gamma} V(0) \cdot \partial_{\gamma} \psi\right]+\operatorname{div}_{\gamma}\left[\partial_{\gamma}(\nabla \psi \cdot V(0))\right] \\
& -\operatorname{div}_{\gamma}\left[\left(\tau^{T}\left(\nabla V(0)+\nabla V(0)^{T}\right) \partial_{\gamma} \psi\right) \cdot \tau\right] \\
= & \partial_{s}\left(\operatorname{div}_{\gamma} V(0)\right) \cdot \partial_{s} \psi+\partial_{s s}\left(\frac{\partial \psi}{\partial n}(V(0) \cdot n)+\partial_{s} \psi(V(0) \cdot \tau)\right)-2 \operatorname{div}_{\gamma}\left[\left(\tau^{T} \nabla V(0) \tau\right) \partial_{s} \psi \tau\right] .
\end{aligned}
$$

Here we used the identity that [30, Lemma 2.63]

$$
\begin{aligned}
\operatorname{div}_{\gamma}\left[\operatorname{div}_{\gamma} V(0) \cdot \partial_{\gamma} \psi\right] & =\partial_{\gamma} \operatorname{div}_{\gamma} V(0) \cdot \partial_{\gamma} \psi+\operatorname{div}_{\gamma} V(0) \operatorname{div}_{\gamma}\left(\partial_{\gamma} \psi\right) \\
& =\partial_{s}\left(\operatorname{div}_{\gamma} V(0)\right) \cdot \partial_{s} \psi+\operatorname{div}_{\gamma} V(0) \Delta_{\gamma} \psi
\end{aligned}
$$

Since $\operatorname{div}_{\gamma} V(0)=\operatorname{div}_{\gamma}[(V(0) \cdot \tau) \tau]-\kappa(V(0) \cdot n)=\partial_{s}(V(0) \cdot \tau)-\kappa(V(0) \cdot n)$,

$$
\begin{aligned}
\left(\left.\Delta_{\gamma} \psi\right|_{\gamma}\right)= & \partial_{s}\left[\partial_{s}(V(0) \cdot \tau)-\kappa(V(0) \cdot n)\right] \cdot \partial_{s} \psi \\
& +\partial_{s s}\left(\frac{\partial \psi}{\partial n}(V(0) \cdot n)+\partial_{s} \psi(V(0) \cdot \tau)\right)-2 \partial_{s}\left[\left(\tau \cdot \partial_{s} V(0)\right) \cdot \partial_{s} \psi\right] \\
= & \partial_{s s}(V(0) \cdot \tau) \cdot \partial_{s} \psi-\partial_{s}[\kappa(V(0) \cdot n)] \cdot \partial_{s} \psi+\partial_{s s}\left(\frac{\partial \psi}{\partial n}(V(0) \cdot n)\right) \\
& +\partial_{s}\left[\partial_{s s} \psi(V(0) \cdot \tau)+\partial_{s} \psi \partial_{s}(V(0) \cdot \tau)\right]-2 \partial_{s}\left[\partial_{s}(\tau \cdot V(0)) \partial_{s} \psi\right]+2 \partial_{s}\left[\left(\partial_{s} \tau \cdot V(0)\right) \partial_{s} \psi\right] \\
= & -\partial_{s}(V(0) \cdot \tau) \cdot \partial_{s s} \psi-\partial_{s}[\kappa(V(0) \cdot n)] \cdot \partial_{s} \psi+\partial_{s s}\left(\frac{\partial \psi}{\partial n}(V(0) \cdot n)\right)+\partial_{s}\left(\partial_{s s} \psi(V(0) \cdot \tau)\right) \\
& +2 \partial_{s}\left[\kappa(n \cdot V(0)) \partial_{s} \psi\right] \\
= & -\partial_{s}[\kappa(V(0) \cdot n)] \cdot \partial_{s} \psi+\partial_{s s}\left(\frac{\partial \psi}{\partial n}(V(0) \cdot n)\right)+\partial_{s s s} \psi(V(0) \cdot \tau)+2 \partial_{s}\left[\kappa(V(0) \cdot n) \partial_{s} \psi\right] .
\end{aligned}
$$

Therefore, by definition,

$$
\begin{aligned}
\left(\left.\Delta_{\gamma} \psi\right|_{\gamma}\right)^{\prime} & =\left(\left.\Delta_{\gamma} \psi\right|_{\gamma}\right) \cdot V(0) \cdot \partial_{\gamma} \Delta_{\gamma} \psi \\
& =-\partial_{s}[\kappa(V(0) \cdot n)] \cdot \partial_{s} \psi+\partial_{s s}\left(\frac{\partial \psi}{\partial n}(V(0) \cdot n)\right)+2 \partial_{s}\left[\kappa(V(0) \cdot n) \partial_{s} \psi\right] \\
& =\partial_{s}[\kappa(V(0) \cdot n)] \cdot \partial_{s} \psi+\partial_{s s}\left(\frac{\partial \psi}{\partial n}(V(0) \cdot n)\right)+2 \kappa(V(0) \cdot n) \partial_{s s} \psi
\end{aligned}
$$


We remark that the first term in C.29 results from the motion of $\gamma$ by $V$ in the normal direction; the second term comes from the change of $\partial_{s} \psi$ due to the change of arclength of $\gamma$; the last term is the directly consequence of the change of arclength.

\section{Shape functionals and auxiliary functions}

Recall that in Section 2.1, we define the normalized net flux induced by the post to be

$$
E(\Omega)=\frac{F(\Omega)}{N(\Omega)}
$$

where

$$
\begin{aligned}
& N(\Omega)=\int_{\Omega} p(x, \theta) \mathrm{d} x \mathrm{~d} \theta+\int_{\gamma}\left[p_{B}^{+}(x)+p_{B}^{-}(x)\right] \mathrm{d} \gamma, \\
& F(\Omega)=\int_{\partial \Omega \cap\left\{x_{2}=b / 2\right\}}-D_{t} \frac{\partial p}{\partial x_{2}}+v_{0} p \sin 2 \pi \theta d A .
\end{aligned}
$$

Suppose we deform the domain $\Omega$ (or equivalently $\Gamma$ ) by the vector field $\tilde{V}(t, x, \theta)$ (or equivalently by the family of flow maps $\left.\left\{\tilde{T}_{t}\right\}_{t \in[0, \varepsilon)}\right)$. The Eulerian derivative of $E(\Omega)$ with respect to $\tilde{V}$ is that

$$
d E(\Omega ; \tilde{V})=\frac{N(\Omega) \cdot d F(\Omega ; \tilde{V})-F(\Omega) \cdot d N(\Omega ; \tilde{V})}{N(\Omega)^{2}}
$$

where

$$
d N(\Omega ; \tilde{V})=\int_{\Omega} p^{\prime}+\int_{\Gamma} p\left(\tilde{V}(0) \cdot n_{\Gamma}\right)+\int_{\gamma}\left(p_{B}^{+\prime}+p_{B}^{-\prime}\right)-\left(p_{B}^{+}+p_{B}^{-}\right) \kappa(V(0) \cdot n),
$$

and

$$
\begin{aligned}
d F(\Omega ; \tilde{V}) & =\int_{\partial \Omega \cap\left\{x_{2}=b / 2\right\}}-D_{t}\left(\left.\frac{\partial p}{\partial x_{2}}\right|_{\partial \Omega \cap\left\{x_{2}=b / 2\right\}}\right)^{\prime}+\left(\left.p\right|_{\partial \Omega \cap\left\{x_{2}=b / 2\right\}}\right)^{\prime} v_{0} \sin 2 \pi \theta d A \\
& =\int_{\partial \Omega \cap\left\{x_{2}=b / 2\right\}}-\left.D_{t} \frac{\partial p^{\prime}}{\partial x_{2}}\right|_{\partial \Omega \cap\left\{x_{2}=b / 2\right\}}+\left.p^{\prime}\right|_{\partial \Omega \cap\left\{x_{2}=b / 2\right\}} v_{0} \sin 2 \pi \theta d A
\end{aligned}
$$

Here we use the assumption that $V$ is zero on $\partial Y$.

According to Hadamard formula (Theorem B.1), we formally write $d E(\Omega ; \tilde{V})$ as

$$
d E(\Omega ; \tilde{V})=\int_{\gamma} G_{E}(x)(V(0) \cdot n) d \gamma,
$$

for some distribution $G_{E}(x)$ defined on $\gamma$. To find out $G_{E}(x)$, we need some auxiliary functions.

Let $\beta=2 \pi \theta-\alpha(x)$ as before. Define $f_{1}(x, \theta)$ in $\Omega$ to be the solution of

$$
\begin{aligned}
& \operatorname{div}\left(D \nabla f_{1}\right)+v_{0}(\cos 2 \pi \theta, \sin 2 \pi \theta, 0) \cdot \nabla f_{1}=1, \quad(x, \theta) \in \Omega, \\
& D_{t} \frac{\partial f_{1}}{\partial n_{\Gamma}}+r_{\mathrm{in}}(\kappa(x))\left[\rho_{+}(\beta)+\rho_{-}(\beta)\right] f_{1}=0, \quad(x, \theta) \in \Gamma,
\end{aligned}
$$

$f_{1}$ satisfies periodic boundary condition on $\partial \Omega \backslash \Gamma$. 
Then by (C.8), (C.11), (D.5)-(D.7) and integration by parts,

$$
\begin{aligned}
\int_{\Omega} p^{\prime}= & \int_{\Omega} p^{\prime}\left[\operatorname{div}\left(D \nabla f_{1}\right)+v_{0}(\cos 2 \pi \theta, \sin 2 \pi \theta, 0) \cdot \nabla f_{1}\right] \\
= & \int_{\Omega}\left[\operatorname{div}\left(D \nabla p^{\prime}\right)-v_{0}(\cos 2 \pi \theta, \sin 2 \pi \theta, 0) \cdot \nabla p^{\prime}\right] \cdot f_{1} \\
& +\int_{\Gamma} D_{t}\left(p^{\prime} \frac{\partial f_{1}}{\partial n_{\Gamma}}-f_{1} \frac{\partial p^{\prime}}{\partial n_{\Gamma}}\right)+v_{0}(\cos 2 \pi \theta, \sin 2 \pi \theta, 0) \cdot n_{\Gamma} \cdot p^{\prime} f_{1} \\
= & 0+\int_{\Gamma}-f_{1}\left[D_{t} \frac{\partial p^{\prime}}{\partial n_{\Gamma}}-v_{0}(\cos 2 \pi \theta, \sin 2 \pi \theta, 0) \cdot n_{\Gamma} p^{\prime}+p^{\prime} r_{\text {in }}\left(\rho_{+}+\rho_{-}\right)\right] \\
= & \int_{\Gamma}-f_{1} \cdot[\text { RHS of C.11] }] .
\end{aligned}
$$

Define $f_{2}^{ \pm}(x)$ on $\gamma$ to be solutions of

$$
-D_{t} \Delta_{\gamma} f_{2}^{ \pm} \mp v_{0} \partial_{s} f_{2}^{ \pm}+r_{\text {out }}(\kappa(x)) f_{2}^{ \pm}=1, \quad x \in \gamma .
$$

By (C.19), D.9) and integration by parts,

$$
\begin{aligned}
\int_{\gamma} p_{B}^{+\prime}+p_{B}^{-\prime}= & \int_{\gamma} p_{B}^{+\prime}\left[-D_{t} \Delta_{\gamma} f_{2}^{+}-v_{0} \partial_{s} f_{2}^{+}+r_{\text {out }}(\kappa(x)) f_{2}^{+}\right] \\
& +\int_{\gamma} p_{B}^{-\prime}\left[-D_{t} \Delta_{\gamma} f_{2}^{-}+v_{0} \partial_{s} f_{2}^{-}+r_{\text {out }}(\kappa(x)) f_{2}^{-}\right] \\
= & \int_{\gamma} f_{2}^{+}\left[-D_{t} \Delta_{\gamma} p_{B}^{+\prime}+v_{0} \partial_{s} p_{B}^{+\prime}+r_{\text {out }}(\kappa(x)) p_{B}^{+\prime}\right] \\
& +\int_{\gamma} f_{2}^{-}\left[-D_{t} \Delta_{\gamma} p_{B}^{-\prime}-v_{0} \partial_{s} p_{B}^{-\prime}+r_{\text {out }}(\kappa(x)) p_{B}^{-\prime}\right] \\
= & \int_{\gamma} f_{2}^{+} \cdot[\text { RHS of C.19), plus case }]+f_{2}^{-} \cdot[\text { RHS of C.19), minus case }] .
\end{aligned}
$$

In order to handle ingredients of $d F(\Omega ; \tilde{V})$ in $(\mathrm{D} .3)$, we start from the following calculation based on C.8,

$$
\begin{aligned}
& \int_{\Omega} v_{0} p^{\prime} \sin 2 \pi \theta \\
= & \int_{\Omega} p^{\prime} \cdot\left[\operatorname{div}\left(D \nabla x_{2}\right)+v_{0}(\cos 2 \pi \theta, \sin 2 \pi \theta, 0) \cdot \nabla x_{2}\right] \\
& -\int_{\Omega} x_{2} \cdot\left[\operatorname{div}\left(D \nabla p^{\prime}\right)-v_{0}(\cos 2 \pi \theta, \sin 2 \pi \theta, 0) \cdot \nabla p^{\prime}\right] \\
= & \int_{\partial \Omega} p^{\prime}\left(D \nabla x_{2}\right) \cdot n_{\partial \Omega}-x_{2}\left(D \nabla p^{\prime}\right) \cdot n_{\partial \Omega}+v_{0} \int_{\partial \Omega} x_{2} p^{\prime}(\cos 2 \pi \theta, \sin 2 \pi \theta, 0) \cdot n_{\partial \Omega} \\
= & \int_{\Gamma} D_{t}\left(p^{\prime} \frac{\partial x_{2}}{\partial n_{\Gamma}}-x_{2} \frac{\partial p^{\prime}}{\partial n_{\Gamma}}\right)-b \int_{\partial \Omega \cap\left\{x_{2}=b / 2\right\}} D_{t} \frac{\partial p^{\prime}}{\partial x_{2}}+v_{0} b \int_{\partial \Omega \cap\left\{x_{2}=b / 2\right\}} p^{\prime} \sin 2 \pi \theta \\
& +v_{0} \int_{\Gamma} x_{2} p^{\prime}(\cos 2 \pi \theta, \sin 2 \pi \theta, 0) \cdot n_{\Gamma} \\
= & b \cdot d F(\Omega ; \tilde{V})+\int_{\Gamma} D_{t}\left(p^{\prime} \frac{\partial x_{2}}{\partial n_{\Gamma}}-x_{2} \frac{\partial p^{\prime}}{\partial n_{\Gamma}}\right)+v_{0} x_{2} p^{\prime} \cos \beta
\end{aligned}
$$

Hence, we define $f_{3}$ on $\Omega$ to be the solution of

$$
\begin{aligned}
& \operatorname{div}\left(D \nabla f_{3}\right)+v_{0}(\cos 2 \pi \theta, \sin 2 \pi \theta, 0) \cdot \nabla f_{3}=v_{0} \sin 2 \pi \theta, \quad(x, \theta) \in \Omega, \\
& D_{t} \frac{\partial\left(f_{3}-x_{2}\right)}{\partial n_{\Gamma}}+r_{\text {in }}(\kappa(x))\left[\rho_{+}+\rho_{-}\right]\left(f_{3}-x_{2}\right)=0, \quad(x, \theta) \in \Gamma,
\end{aligned}
$$

$f_{3}$ satisfies periodic boundary condition on $\partial \Omega \backslash \Gamma$. 
Then

$$
\begin{aligned}
b \cdot d F(\Omega ; \tilde{V})= & \int_{\Omega} v_{0} p^{\prime} \sin 2 \pi \theta-\int_{\Gamma} D_{t}\left(p^{\prime} \frac{\partial x_{2}}{\partial n_{\Gamma}}-x_{2} \frac{\partial p^{\prime}}{\partial n_{\Gamma}}\right)+v_{0} x_{2} p^{\prime} \cos \beta \\
= & \int_{\Omega} p^{\prime}\left[\operatorname{div}\left(D \nabla f_{3}\right)+v_{0}(\cos 2 \pi \theta, \sin 2 \pi \theta, 0) \cdot \nabla f_{3}\right] \\
& -\int_{\Gamma} D_{t}\left(p^{\prime} \frac{\partial x_{2}}{\partial n_{\Gamma}}-x_{2} \frac{\partial p^{\prime}}{\partial n_{\Gamma}}\right)+v_{0} x_{2} p^{\prime} \cos \beta \\
= & \int_{\Omega} f_{3}\left[\operatorname{div}\left(D \nabla p^{\prime}\right)-v_{0}(\cos 2 \pi \theta, \sin 2 \pi \theta, 0) \cdot \nabla p^{\prime}\right] \\
& +\int_{\Gamma} D_{t}\left(p^{\prime} \frac{\partial f_{3}}{\partial n_{\Gamma}}-f_{3} \frac{\partial p^{\prime}}{\partial n_{\Gamma}}\right)+v_{0} f_{3} p^{\prime} \cos \beta \\
& -\int_{\Gamma} D_{t}\left(p^{\prime} \frac{\partial x_{2}}{\partial n_{\Gamma}}-x_{2} \frac{\partial p^{\prime}}{\partial n_{\Gamma}}\right)+v_{0} x_{2} p^{\prime} \cos \beta \\
= & 0+\int_{\Gamma} D_{t}\left(p^{\prime} \frac{\partial\left(f_{3}-x_{2}\right)}{\partial n_{\Gamma}}-\left(f_{3}-x_{2}\right) \frac{\partial p^{\prime}}{\partial n_{\Gamma}}\right)+v_{0}\left(f_{3}-x_{2}\right) p^{\prime} \cos \beta \\
= & \int_{\Gamma}-\left(f_{3}-x_{2}\right)\left(r_{\mathrm{in}}(\kappa(x))\left[\rho_{+}+\rho_{-}\right] p^{\prime}+D_{t} \frac{\partial p^{\prime}}{\partial n_{\Gamma}}-v_{0} p^{\prime} \cos \beta\right) \\
= & \int_{\Gamma}-\left(f_{3}-x_{2}\right) \cdot[\operatorname{RHS} \text { of }[\mathrm{C.11}] .
\end{aligned}
$$

In summary, by (D.1), (D.2), (D.3), (D.8), D.10) and (D.15),

$$
\begin{aligned}
d E(\Omega ; \tilde{V})= & \int_{\Gamma}\left(\frac{1}{b N(\Omega)}\left(x_{2}-f_{3}\right)+\frac{F(\Omega)}{N(\Omega)^{2}} f_{1}\right) \cdot[\text { RHS of [C.11] ] } \\
& -\frac{F(\Omega)}{N(\Omega)^{2}}\left[\int _ { \gamma } f _ { 2 } ^ { + } \cdot \left[\text { RHS of }(\mathrm{C} .19), \text { plus case] }+f_{2}^{-} \cdot[\text { RHS of } \text { C.19), minus case] }\right.\right. \\
& \left.+\int_{\Gamma} p\left(\tilde{V}(0) \cdot n_{\Gamma}\right)-\int_{\gamma}\left(p_{B}^{+}+p_{B}^{-}\right) \kappa(V(0) \cdot n)\right] .
\end{aligned}
$$

We remark that once the linear dependence of $\left(p^{\prime}, p_{B}^{+\prime}, p_{B}^{-\prime}\right)$ on $V(0) \cdot n$ (or equivalently $\tilde{V}(0) \cdot n_{\Gamma}$ ) is established, by the formulae for $\alpha^{\prime}(x)$ and $\kappa^{\prime}(x)$ (C.24) and (C.26) respectively), $d E(\Omega ; \tilde{V})$ is a linear functional of $V(0) \cdot n$. Then the distribution $G_{E}(x)$ in (D.4) is well-defined.

\section{E Linear dependence of $\left(p^{\prime}, p_{B}^{+\prime}, p_{B}^{-\prime}\right)$ on $V(0) \cdot n$}

In this section, we shall show that we can indeed define a linear map from $V(0) \cdot n$ (or equivalent $\left.\tilde{V}(0) \cdot n_{\Gamma}\right)$ to $\left(p^{\prime}, p_{B}^{+\prime}, p_{B}^{-\prime}\right)$.

Once $\left(p, p_{B}^{+}, p_{B}^{-}\right)$has been solved via the method in Section $\mathrm{A}$, they can be viewed as known functions in the coupled system (C.8), C.11) and (C.19) for $p^{\prime}$ and $p_{B}^{ \pm \prime}$. We simply rewrite the equations for $p^{\prime}$ and $p_{B}^{ \pm \prime}$ as follows

$$
\begin{aligned}
& \operatorname{div}\left(D \nabla p^{\prime}\right)=v_{0}(\cos 2 \pi \theta, \sin 2 \pi \theta, 0) \cdot \nabla p^{\prime}, \quad(x, \theta) \in \Omega, \\
& D_{t} \frac{\partial p^{\prime}}{\partial n_{\Gamma}}-v_{0} p^{\prime} \cos \beta+r_{\mathrm{in}}\left(\rho_{+}+\rho_{-}\right) p^{\prime}=r_{\text {out }} \cdot 2 \pi\left(\tau_{+} p_{B}^{+\prime}+\tau_{-} p_{B}^{-\prime}\right)+\mathcal{L}_{1}\left(\tilde{V}(0) \cdot n_{\Gamma}\right), \quad(x, \theta) \in \Gamma, \\
& -D_{t} \Delta_{\gamma} p_{B}^{ \pm \prime} \pm v_{0} \partial_{s} p_{B}^{ \pm \prime}+r_{\text {out }} p_{B}^{ \pm \prime}=\mathcal{L}_{2}^{ \pm}(V(0) \cdot n)+\int_{0}^{1} r_{\text {in }} \rho_{ \pm} p^{\prime} d \theta, \quad x \in \gamma,
\end{aligned}
$$

$p^{\prime}$ satisfies periodic boundary condition on $\partial \Omega \backslash \Gamma$. 
Here $\mathcal{L}_{1}$ and $\mathcal{L}_{2}^{ \pm}$are linear operators depending on all the known functions including $p$ and $p_{B}^{ \pm}$. To be more precise,

$$
\begin{aligned}
\mathcal{L}_{1}\left(\tilde{V}(0) \cdot n_{\Gamma}\right)= & \frac{d r_{\text {out }}}{d \kappa}(\kappa(x)) \kappa^{\prime}(x)\left[2 \pi \tau_{+}(\beta) p_{B}^{+}(x)+2 \pi \tau_{-}(\beta) p_{B}^{-}(x)\right] \\
& +r_{\text {out }}(\kappa(x)) \cdot 2 \pi\left(-\alpha^{\prime}(x)\right) \cdot\left[\frac{d \tau_{+}}{d \beta}(\beta) p_{B}^{+}(x)+\frac{d \tau_{-}}{d \beta}(\beta) p_{B}^{-}(x)\right] \\
& -\frac{d r_{\text {in }}}{d \kappa}(\kappa(x)) \kappa^{\prime}(x)\left(\rho_{+}(\beta)+\rho_{-}(\beta)\right) p \\
& -r_{\text {in }}(\kappa(x))\left(-\alpha^{\prime}(x)\right) \cdot\left[\frac{d \rho_{+}}{d \beta}(\beta)+\frac{d \rho_{-}}{d \beta}(\beta)\right] p \\
& -r_{\text {in }}\left(\rho_{+}+\rho_{-}\right) \frac{\partial p}{\partial n_{\Gamma}}\left(\tilde{V}(0) \cdot n_{\Gamma}\right)-\kappa\left(\tilde{V}(0) \cdot n_{\Gamma}\right)\left(g-r_{\text {in }} p\left(\rho_{+}+\rho_{-}\right)\right) \\
& +\operatorname{div}_{\Gamma}\left[\left(\tilde{V}(0) \cdot n_{\Gamma}\right) D \nabla_{\Gamma} p-v_{0}\left(\tilde{V}(0) \cdot n_{\Gamma}\right) p \mathbb{P}_{\Gamma}(\cos 2 \pi \theta, \sin 2 \pi \theta, 0)\right],
\end{aligned}
$$

and

$$
\begin{aligned}
\mathcal{L}_{2}^{ \pm}(V(0) \cdot n)= & \int_{0}^{1} \frac{d r_{\text {in }}}{d \kappa}(\kappa(x)) \kappa^{\prime}(x) \rho_{ \pm} p(x, \theta)+r_{\text {in }}(\kappa(x)) \frac{d \rho_{ \pm}}{d \beta}(\beta)\left(-\alpha^{\prime}(x)\right) p(x, \theta) d \theta \\
& +\int_{0}^{1} r_{\text {in }}(\kappa(x)) \rho_{ \pm} \frac{\partial p}{\partial n_{\Gamma}}(x, \theta)(V(0) \cdot n)-\kappa(V(0) \cdot n) r_{\text {in }} \rho_{ \pm} p(x, \theta) d \theta \\
& -\frac{d r_{\text {out }}}{d \kappa}(\kappa(x)) \kappa^{\prime}(x) p_{B}^{ \pm}+D_{t} \partial_{s}\left[\kappa(V(0) \cdot n) \partial_{s} p_{B}^{ \pm}\right]+r_{\text {out }}(\kappa(x)) \kappa(V(0) \cdot n) p_{B}^{ \pm} .
\end{aligned}
$$

Note that by (C.24) and (C.26), $\alpha^{\prime}(x)$ and $\kappa^{\prime}(x)$ are linear in $V(0) \cdot n$.

Remark E.1. It could be shown that

$$
\int_{\Gamma} \mathcal{L}_{1}\left(\tilde{V}(0) \cdot n_{\Gamma}\right)+\int_{\gamma} \mathcal{L}_{2}^{+}(V(0) \cdot n)+\mathcal{L}_{2}^{-}(V(0) \cdot n)=0 .
$$

Indeed, by C.11 and C.19,

$$
\begin{aligned}
\int_{\Gamma} \mathcal{L}_{1}\left(\tilde{V}(0) \cdot n_{\Gamma}\right)= & \int_{\gamma} \frac{d r_{\text {out }}}{d \kappa}(\kappa(x)) \kappa^{\prime}(x)\left(p_{B}^{+}+p_{B}^{-}\right)-\kappa(V(0) \cdot n) r_{\text {out }}(\kappa(x))\left(p_{B}^{+}+p_{B}^{-}\right) \\
& +\int_{\Gamma}\left\{-\frac{d r_{\text {in }}}{d \kappa}(\kappa(x)) \kappa^{\prime}(x)\left(\rho_{+}(\beta)+\rho_{-}(\beta)\right) p\right. \\
& \left.-r_{\text {in }}\left(\rho_{+}+\rho_{-}\right) \frac{\partial p}{\partial n_{\Gamma}}\left(\tilde{V}(0) \cdot n_{\Gamma}\right)+\kappa\left(\tilde{V}(0) \cdot n_{\Gamma}\right) r_{\text {in }} p\left(\rho_{+}+\rho_{-}\right)\right\},
\end{aligned}
$$

and

$$
\begin{aligned}
\int_{\gamma} \mathcal{L}_{2}^{ \pm}(V(0) \cdot n)= & \int_{\Gamma}\left\{\frac{d r_{\text {in }}}{d \kappa}(\kappa(x)) \kappa^{\prime}(x) \rho_{ \pm} p(x, \theta)\right. \\
& \left.+r_{\text {in }}(\kappa(x)) \rho_{ \pm} \frac{\partial p}{\partial n_{\Gamma}}(x, \theta)(V(0) \cdot n)-\kappa(V(0) \cdot n) r_{\text {in }} \rho_{ \pm} p(x, \theta)\right\} \\
& +\int_{\gamma}-\frac{d r_{\text {out }}}{d \kappa}(\kappa(x)) \kappa^{\prime}(x) p_{B}^{ \pm}+r_{\text {out }}(\kappa(x)) \kappa(V(0) \cdot n) p_{B}^{ \pm} .
\end{aligned}
$$

Then (E.7) follows immediately.

We may follow exactly the same scheme as in Section A to solve the system E.1)-(E.4). To be 
more precise, we consider the following equations with given $p_{B}^{ \pm \prime}$ and $V(0) \cdot n$

$$
\begin{aligned}
& \operatorname{div}\left(D \nabla \tilde{p}^{\prime}\right)=v_{0}(\cos 2 \pi \theta, \sin 2 \pi \theta, 0) \cdot \nabla \tilde{p}^{\prime}, \quad(x, \theta) \in \Omega, \\
& D_{t} \frac{\partial \tilde{p}^{\prime}}{\partial n_{\Gamma}}-v_{0} \tilde{p}^{\prime} \cos \beta+r_{\text {in }}\left(\rho_{+}+\rho_{-}\right) \tilde{p}^{\prime}=r_{\text {out }} \cdot 2 \pi\left(\tau_{+} p_{B}^{+\prime}+\tau_{-} p_{B}^{-\prime}\right)+\mathcal{L}_{1}\left(\tilde{V}(0) \cdot n_{\Gamma}\right), \quad(x, \theta) \in \Gamma \\
& -D_{t} \Delta_{\gamma} \tilde{p}_{B}^{ \pm \prime} \pm v_{0} \partial_{s} \tilde{p}_{B}^{ \pm \prime}+r_{\text {out }} \tilde{p}_{B}^{ \pm \prime}=\mathcal{L}_{2}^{ \pm}(V(0) \cdot n)+\int_{0}^{1} r_{\text {in }} \rho_{ \pm} \tilde{p}^{\prime} d \theta, \quad x \in \gamma,
\end{aligned}
$$

$\tilde{p}^{\prime}$ satisfies periodic boundary condition on $\partial \Omega \backslash \Gamma$.

By solving the above equations, we can establish the linear map $K^{\prime}:\left(p_{B}^{+}, p_{B}^{-}, \tilde{V}(0) \cdot n_{\Gamma}\right) \mapsto\left(\tilde{p}_{B}^{+}, \tilde{p}_{B}^{-}\right)$. We already know that

$$
K^{\prime}\left[p_{B}^{+\prime}, p_{B}^{-\prime}, 0\right]=\left(\tilde{p}_{B}^{+\prime}, \tilde{p}_{B}^{-\prime}\right)=K\left[p_{B}^{+\prime}, p_{B}^{-\prime}\right]
$$

where $K$ is defined in Section A. Hence, by linear superposition principle, it suffices to study

$$
S(V(0) \cdot n) \triangleq K^{\prime}[0,0, V(0) \cdot n],
$$

which is a linear map. Once $S$ is found out, solving (C.8, C.11 and C.19 is equivalent to solving

$$
(K-I d)\left[p_{B}^{+\prime}, p_{B}^{-\prime}\right]=-S(V(0) \cdot n),
$$

Remark E.2. By Remark A.2, a necessary condition for E.13 to be solvable is that

$$
\left\langle\left(r_{\text {out }}, r_{\text {out }}\right), S(V(0) \cdot n)\right\rangle=0,
$$

where $\langle\cdot, \cdot\rangle$ again denotes the inner product of $L^{2}(\gamma) \times L^{2}(\gamma)$. This is automatically satisfied. Indeed, by (E.8)-E.11, we proceed as in A.8,

$$
\begin{aligned}
& \left\langle\left(r_{\text {out }}, r_{\text {out }}\right), S(V(0) \cdot n)\right\rangle \\
= & \int_{\gamma} r_{\text {out }}\left(\tilde{p}_{B}^{+}+\tilde{p}_{B}^{-}\right) \\
= & \int_{\gamma} \mathcal{L}_{2}^{+}(V(0) \cdot n)+\mathcal{L}_{2}^{-}(V(0) \cdot n)+\int_{\Gamma} r_{\text {in }} \tilde{p}^{\prime}\left(\rho_{+}+\rho_{-}\right) \\
= & \int_{\gamma} \mathcal{L}_{2}^{+}(V(0) \cdot n)+\mathcal{L}_{2}^{-}(V(0) \cdot n)+\int_{\Gamma}-D_{t} \frac{\partial \tilde{p}^{\prime}}{\partial n_{\Gamma}}+v_{0} \tilde{p}^{\prime} \cos \beta+\mathcal{L}_{1}\left(\tilde{V}(0) \cdot n_{\Gamma}\right) \\
= & \int_{\gamma} \mathcal{L}_{2}^{+}(V(0) \cdot n)+\mathcal{L}_{2}^{-}(V(0) \cdot n)+\int_{\Gamma} \mathcal{L}_{1}\left(\tilde{V}(0) \cdot n_{\Gamma}\right) \\
& -\int_{\Omega} \operatorname{div}\left(D \nabla \tilde{p}^{\prime}\right)-v_{0}(\cos 2 \pi \theta, \sin 2 \pi \theta, 0) \cdot \nabla \tilde{p}^{\prime} \\
= & 0
\end{aligned}
$$

In the last line, we used Remark E.1 and E.8.

Suppose E.13) is solvable, i.e. $S(V(0) \cdot n)$ is in the range of $(K-I d)$. It will have infinitely many solutions since $(K-I d)$ has a nontrivial kernel. Recall that by (A.7), any element in the kernel of $(K-I d)$ is a solution of the coupled system (2.7)-(2.11) for $p$ and $p_{B}^{ \pm}$. However, we shall show that any two solutions of E.13), whose difference is in the kernel of $(K-I d)$, will give the same $d E(\Omega ; \tilde{V})$ in $(\mathrm{D} .16)$. For given $V(0) \cdot n$ and given the unique positive solution $\left(p, p_{B}^{+}, p_{B}^{-}\right)$of 2.7)-2.11) satisfying (2.12), assume $\left(p_{k}^{\prime}, p_{B, k}^{+}{ }^{\prime}, p_{B, k}^{-}{ }^{\prime}\right), k=1,2$, to be two solutions of (E.13). By (A.7) and E.13), $\left(q, q_{B}^{+}, q_{B}^{-}\right) \triangleq\left(p_{1}^{\prime}-p_{2}^{\prime}, p_{B, 1}^{+}{ }^{\prime}-p_{B, 2}^{+}{ }^{\prime}, p_{B, 1}^{-}{ }^{\prime}-p_{B, 2}^{-}{ }^{\prime}\right)$ is a solution of 2.7)-2.11. In particular, it is a multiple of $\left(p, p_{B}^{+}, p_{B}^{-}\right)$by the assumption that $(K-I d)$ has only one-dimensional 
kernel (see Remark A.1). Let $d E_{i}(\Omega ; \tilde{V})$ be the Eulerian derivative of $E(\Omega)$ represented in terms of the solutions $\left(p_{k}^{\prime}, p_{B, k}^{+}{ }^{\prime}, p_{B, k}^{-}{ }^{\prime}\right)$ respectively. By (D.16),

$$
\begin{aligned}
& d E_{1}(\Omega ; \tilde{V})-d E_{2}(\Omega ; \tilde{V}) \\
= & \int_{\Gamma}\left(\frac{1}{b N(\Omega)}\left(x_{2}-f_{3}\right)+\frac{F(\Omega)}{N(\Omega)^{2}} f_{1}\right) \cdot r_{\text {out }}(\kappa) \cdot 2 \pi\left[\tau_{+}(\beta) q_{B}^{+}(x)+\tau_{-}(\beta) q_{B}^{-}(x)\right] \\
& -\left.\frac{F(\Omega)}{N(\Omega)^{2}} \int_{\Gamma} q\right|_{\Gamma} r_{\mathrm{in}}(\kappa)\left[f_{2}^{+}(x) \rho_{+}(\beta)+f_{2}^{-} \rho_{-}(\beta)\right] \\
= & \int_{\Gamma}\left(\frac{1}{b N(\Omega)}\left(x_{2}-f_{3}\right)+\frac{F(\Omega)}{N(\Omega)^{2}} f_{1}\right)\left(r_{\mathrm{in}}(\kappa(x))\left[\rho_{+}+\rho_{-}\right] q+D_{t} \frac{\partial q}{\partial n_{\Gamma}}-v_{0} q \cos \beta\right) \\
& -\frac{F(\Omega)}{N(\Omega)^{2}} \int_{\gamma} f_{2}^{+}(x)\left(-D_{t} \Delta_{\gamma} q_{B}^{+}+v_{0} \partial_{\gamma} p_{B}^{+}+r_{\text {out }} p_{B}^{+}\right) \\
& -\frac{F(\Omega)}{N(\Omega)^{2}} \int_{\gamma} f_{2}^{-}(x)\left(-D_{t} \Delta_{\gamma} q_{B}^{-}-v_{0} \partial_{\gamma} p_{B}^{-}+r_{\text {out }} p_{B}^{-}\right) .
\end{aligned}
$$

Here we used the assumption that $\left(q, q_{B}^{+}, q_{B}^{-}\right)$satisfies 2.7)-(2.11). Following the derivation of (D.11) and (D.15) in the reverse direction, with $p^{\prime}$ replaced by $q$, we find that

$$
\begin{aligned}
& \int_{\Gamma}\left(x_{2}-f_{3}\right) \cdot r_{\text {out }}(\kappa) \cdot 2 \pi\left[\tau_{+}(\beta) q_{B}^{+}(x)+\tau_{-}(\beta) q_{B}^{-}(x)\right] \\
= & -b \int_{\partial \Omega \cap\left\{x_{2}=b / 2\right\}} D_{t} \frac{\partial q}{\partial x_{2}}+v_{0} b \int_{\partial \Omega \cap\left\{x_{2}=b / 2\right\}} q \sin 2 \pi \theta=b F(\Omega ; q),
\end{aligned}
$$

where $F(\Omega ; q)$ is the net flux generated by the distribution $q$ in $\Omega$. Similarly, proceeding as in (D.8) and $(\mathrm{D} .10)$ in the reverse direction, with $\left(p^{\prime}, p_{B}^{+\prime}, p_{B}^{-\prime}\right)$ replaced by $\left(q, q_{B}^{+}, q_{B}^{-}\right)$, we find that

$$
\begin{aligned}
& \int_{\Gamma} f_{1}\left(r_{\mathrm{in}}(\kappa(x))\left[\rho_{+}+\rho_{-}\right] q+D_{t} \frac{\partial q}{\partial n_{\Gamma}}-v_{0} q \cos \beta\right) \\
& -\int_{\gamma} f_{2}^{+}(x)\left(-D_{t} \Delta_{\gamma} q_{B}^{+}+v_{0} \partial_{\gamma} p_{B}^{+}+r_{\mathrm{out}} p_{B}^{+}\right)-\int_{\gamma} f_{2}^{-}(x)\left(-D_{t} \Delta_{\gamma} q_{B}^{-}-v_{0} \partial_{\gamma} p_{B}^{-}+r_{\mathrm{out}} p_{B}^{-}\right) \\
= & -\int_{\Omega} q-\int_{\gamma}\left(q_{B}^{+}+q_{B}^{-}\right)=N\left(\Omega ;\left(q, q_{B}^{ \pm}\right)\right) .
\end{aligned}
$$

Hence, combining (E.14), (E.15) and (E.16), we find

$$
d E_{1}(\Omega ; \tilde{V})-d E_{2}(\Omega ; \tilde{V})=\frac{F(\Omega ; q)}{N(\Omega)}-\frac{F(\Omega) N\left(\Omega ;\left(q, q_{B}^{ \pm}\right)\right)}{N(\Omega)^{2}}=\frac{N\left(\Omega ;\left(q, q_{B}^{ \pm}\right)\right)}{N(\Omega)}\left[E\left(\Omega ;\left(q, q_{B}^{ \pm}\right)\right)-E(\Omega)\right] .
$$

Since $\left(q, q_{B}^{+}, q_{B}^{-}\right)$is a multiple of $\left(p, p_{B}^{+}, p_{B}^{-}\right)$, then $N\left(\Omega ;\left(q, q_{B}^{ \pm}\right)\right)=0$ or $E\left(\Omega ;\left(q, q_{B}^{ \pm}\right)\right)=E(\Omega)$, which implies that $d E_{1}(\Omega ; \tilde{V})=d E_{2}(\Omega ; \tilde{V})$.

Therefore, it suffices to consider the solution $\left(p_{B}^{+}, p_{B}^{-}\right)$as an element in the quotient space $\operatorname{dom}(K-I d) / \operatorname{ker}(K-I d)$; it is well-defined and is linear in $V(0) \cdot n$. We are thus able to define a linear map from $V(0) \cdot n$ to $\left(p^{\prime}, p_{B}^{+\prime}, p_{B}^{-\prime}\right)$. By $(\mathrm{D} .16), d E(\Omega ; \tilde{V})$ is indeed a linear functional of $V(0) \cdot n$. Therefore, $G_{E}(x)$ in (D.4) is well-defined.

\section{F Numerical methods for the shape optimization}

As is discussed in Remark B.2, $E(\Omega)$ increases fastest if $\gamma$ evolves in the direction of $V$ such that $V \cdot n=G_{E}(x)$ for $x \in \gamma$. Note that only the value of $V(t, x)$ for $x \in \gamma$ and $t=0$ will be used in representing $d E(\Omega, \tilde{V})$. We shall omit the $t$-dependence of $V$ and $\tilde{V}$ in the sequel. 
In order to apply steepest ascent method, the explicit form of $G_{E}(x)$ is needed, which means a vectorial representation of $G_{E}$ in the discretized case. Therefore, the numerical method below aims at first establishing a discretized representation of the linear operator $S$ defined in E.12, and then finding out the map from $V \cdot n$ to $\left(p^{\prime}, p_{B}^{+\prime}, p_{B}^{-\prime}\right)$ and $d E(\Omega ; \tilde{V})$ in its discrete form. The vectorial representation of $G_{E}$ then follows. It goes as follows:

1. Fix $\gamma$. We represent $\gamma$ using the same $N$ points $\left\{x_{1}, \cdots, x_{N}\right\} \triangleq X$ as in Section A We also use A.9 - A.11 to evaluate $\alpha(x), n(x)$ and $\kappa(x)$ on the grid points in $X$. In the $\theta$-direction, $[0,1]$ is again discretized evenly using $M$ points $\left\{\theta_{1}, \cdots, \theta_{M}\right\}$ defined in Section A. Let $X_{\Gamma}$ be defined as in A.12.

2. Through the recipe in Section A, matrices $U$ and $W$ can be constructed and a normalized solution $\left(p, p_{B}^{+}, p_{B}^{-}\right)$can be found out. In particular, values of $p$ have been determined on the boundary grid $X_{\Gamma}$ on $\Gamma$, while $p_{B}^{ \pm}$have been solved on $X$. Unnormalized net flux $F(\Omega)$ and the normalizing factor $N(\Omega)=1$ could be evaluated.

Calculate the pseudo-inverse of $(W-U)$, denoted by $(W-U)^{\dagger}$, by singular value decomposition 41. Recall that the pseudo-inverse have the following properties: $(W-U)^{\dagger}(W-U) \xi=\xi$ for $\forall \xi \perp \operatorname{ker}(W-U) \in \mathbb{R}^{2 N} ;$ and range $(W-U)^{\dagger} \perp \operatorname{ker}(W-U)$.

3. Let $\left\{\mathbf{y}_{1}, \cdots, \mathbf{y}_{N}\right\}$ be an orthogonal basis of $\mathbb{R}^{N}$, which are column vectors. They form a basis of all possible vectorial representations of $V \cdot n$ on the grid points in $X$. Denote $Y=\left(\mathbf{y}_{1}, \cdots, \mathbf{y}_{N}\right)$. We take $Y=U_{0}^{T}$ defined in A.13) and A.14.

4. Let $V_{i}$ be a perturbation vector field defined on $\gamma$, whose values on the grid points in $X$ are given by

$$
\left(V_{i}\left(x_{1}\right), \cdots, V_{i}\left(x_{N}\right)\right)^{T}=\left(y_{i, 1} \cdot n\left(x_{1}\right), \cdots, y_{i, N} \cdot n\left(x_{N}\right)\right)^{T} .
$$

Here $y_{i, j} \in \mathbb{R}$ is the $j$-th component of $\mathbf{y}_{i}$. Hence,

$$
\left(\left[V_{i} \cdot n\right]\left(x_{1}\right), \cdots,\left[V_{i} \cdot n\right]\left(x_{N}\right)\right)^{T}=\mathbf{y}_{i} .
$$

We use the central difference scheme to calculate $\partial_{s}\left[V_{i} \cdot n\right]$ and $\partial_{s s}\left[V_{i} \cdot n\right]$ on points in $X$,

$$
\begin{aligned}
\partial_{s}\left[V_{i} \cdot n\right]\left(x_{j}\right) & =\frac{1}{2 \Delta s}\left(y_{i, j+1}-y_{i, j-1}\right), \\
\partial_{s s}\left[V_{i} \cdot n\right]\left(x_{j}\right) & =\frac{1}{(\Delta s)^{2}}\left(y_{i, j+1}-2 y_{i, j}+y_{i, j-1}\right) .
\end{aligned}
$$

Then $\alpha^{\prime}\left(x_{j}\right)$ 's and $\kappa^{\prime}\left(x_{j}\right)$ 's are evaluated using C.12 and C.13 respectively.

5. Given values of $p$ on $X_{\Gamma}$ and values of $p_{B}^{ \pm}$on $X$, we evaluate $\mathcal{L}_{1}\left(\tilde{V}_{i} \cdot n_{\Gamma}\right)$ on $X_{\Gamma}$ by E.5). The tangential divergence $\operatorname{div}_{\Gamma}$ is calculated by central difference scheme in $\theta$ - and $s$-coordinates, where $s$ is the arclength parameter of $\gamma$. Periodic boundary condition of $p$ in the $\theta$-direction on $\Gamma$ is used here. Similarly, we calculate $\mathcal{L}_{2}^{ \pm}\left(V_{i} \cdot n\right)$ on $X$ by E.6).

6. With $\mathcal{L}_{1}\left(\tilde{V}_{i} \cdot n_{\Gamma}\right)$ specified on $X_{\Gamma}$, we solve the following subproblem by COMSOL

$$
\begin{aligned}
& \operatorname{div}\left(D \nabla q_{i}^{\prime}\right)=v_{0}(\cos 2 \pi \theta, \sin 2 \pi \theta, 0) \cdot \nabla q_{i}^{\prime}, \quad(x, \theta) \in \Omega, \\
& D_{t} \frac{\partial q_{i}^{\prime}}{\partial n_{\Gamma}}-v_{0} q_{i}^{\prime} \cos \beta+r_{\text {in }}\left(\rho_{+}+\rho_{-}\right) q_{i}^{\prime}=\mathcal{L}_{1}\left(\tilde{V}_{i} \cdot n_{\Gamma}\right), \quad(x, \theta) \in \Gamma, \\
& q_{i}^{\prime} \text { satisfies periodic boundary condition on } \partial \Omega \backslash \Gamma .
\end{aligned}
$$

We obtain the Dirichlet boundary data of $q_{i}^{\prime}$ on the set $X_{\Gamma}$. 
7. Now consider the equation for $q_{B, i}^{ \pm}$

$$
-D_{t} \Delta_{\gamma} q_{B, i}^{ \pm}{ }^{\prime} \pm v_{0} \partial_{s} q_{B, i}^{ \pm}{ }^{\prime}+r_{\text {out }} q_{B, i}^{ \pm}{ }^{\prime}=\mathcal{L}_{2}^{ \pm}\left(V_{i} \cdot n\right)+\int_{0}^{1} r_{\text {in }} \rho_{ \pm} q_{i}^{\prime} d \theta, \quad x \in \gamma .
$$

The integral on the right hand side is again evaluated on $X$ using the trapezoidal rule as in A.17, with $\tilde{p}_{i}$ replaced by $q_{i}^{\prime}$. Then we solve $\mathrm{F.2}$ for $q_{B, i}^{ \pm}$by the finite difference scheme with grid points $X$. We denote

$$
\left(q_{B, i}^{+}{ }^{\prime}\left(x_{1}\right), \cdots, q_{B, i}^{+}{ }^{\prime}\left(x_{N}\right), q_{B, i}^{-}{ }^{\prime}\left(x_{1}\right), \cdots, q_{B, i}^{-}{ }^{\prime}\left(x_{N}\right)\right)^{T} \triangleq \mathbf{z}_{i} .
$$

In this way, we obtain $\mathbf{z}_{i}=S \mathbf{y}_{i}$, where $S$, with abuse of notations, is a discrete representation of the operator $S$ defined in (E.12). $S$ is not explicitly represented here.

8. Repeating Step 4- Step7for $i=1, \cdots, N$, we form an $2 N \times N$ matrix $Z=\left(\mathbf{z}_{1}, \cdots, \mathbf{z}_{N}\right)=S Y$.

9. Given $V_{i} \cdot n$, values of the corresponding $\left(p_{B, i}^{+}{ }^{\prime}, p_{B, i}^{-}{ }^{\prime}\right)$ on $X$ are given by

$$
\left(p_{B, i}^{+}{ }^{\prime}\left(x_{1}\right), \cdots, p_{B, i}^{+}{ }^{\prime}\left(x_{N}\right), p_{B, i}^{-}{ }^{\prime}\left(x_{1}\right), \cdots, p_{B, i}^{-}{ }^{\prime}\left(x_{N}\right)\right)^{T}=-U(W-U)^{\dagger} \mathbf{z}_{i} .
$$

Indeed, since $U$ is invertible, we assume $\left(p_{B, i}^{+}{ }^{\prime}\left(x_{1}\right), \cdots, p_{B, i}^{+}{ }^{\prime}\left(x_{N}\right), p_{B, i}{ }^{\prime}\left(x_{1}\right), \cdots, p_{B, i}^{-}{ }^{\prime}\left(x_{N}\right)\right)^{T}=$ $U \eta_{i}$ for some $\eta_{i} \in \mathbb{R}^{2 N}$. By (E.13), $\left(K-I_{2 N}\right) U \eta_{i}=-S \mathbf{y}_{i}$, i.e. $(W-U) \eta_{i}=-S \mathbf{y}_{i}$. By the discussion in Section $\mathrm{E}$, it suffices to solve for $U \eta_{i}$ such that $U \eta_{i} \perp \operatorname{ker}\left(K-I_{2 N}\right)$, i.e. $\eta_{i} \perp \operatorname{ker}\left(\left(K-I_{2 N}\right) U\right)=\operatorname{ker}(W-U)$. Hence, $\eta_{i}=-(W-U)^{\dagger} S \mathbf{y}_{i}$, which justifies $($ F.3).

10. To this end, we solve for $p$ through (E.1), E.2 and (E.4) by COMSOL. The values of $V_{i}(0) \cdot n$ and $\left(p_{B, i}^{+}, p_{B, i}{ }^{\prime}\right)$ on $X$ are used in $(E .2)$. In this way, the Dirichlet data of $p^{\prime}$ on $X_{\Gamma}$ is obtained.

11. Use COMSOL to solve for $f_{1}$ and $f_{3}$ by (D.5)-(D.7) and (D.12)-(D.14), respectively. Solve for $f_{2}^{ \pm}$by applying finite difference scheme to D.9 with grid points in $X$.

12. Finally, we evaluate $d E\left(\Omega ; \tilde{V}_{i}\right)$ using D.16) for $i=1, \cdots, N$. The integrals in D.16) are evaluated by the trapezoidal rule. Then under discretization, the vectorial representation of the shape gradient $G_{E}(x)$ in $(\mathrm{D} .4)$, denoted by $\mathbf{G} \in \mathbb{R}^{N}$, is given by

$$
\mathbf{G}=\sum_{i=1}^{N} \frac{d E\left(\Omega ; \tilde{V}_{i}\right)}{\left(\mathbf{y}_{i} \cdot \mathbf{y}_{i}\right) \Delta s} \mathbf{y}_{i} .
$$

We derive this from $d E\left(\Omega ; \tilde{V}_{i}\right)=\left(\mathbf{G} \cdot \mathbf{y}_{i}\right) \Delta s$ and the orthogonality of $\left\{\mathbf{y}_{i}\right\}$.

13. We apply steepest ascent method with fixed step-size to evolve $\gamma$. Choose $\varepsilon$ to be suitably small, and calculate

$$
\tilde{x}_{j}=x_{j}+\varepsilon n\left(x_{j}\right) \cdot G_{j},
$$

where $G_{j}$ is the $j$-th component of G. Note that by Hadamard formula (D.4), only the normal component of the perturbation vector field matters. The new configuration of the post boundary, denoted by $\tilde{\gamma}$, is then given by $\left\{\tilde{x}_{j}\right\}$ and a spline interpolation. In the Remark F.1 below, we shall propose a better way of evolving $\gamma$.

14. Repeat all the above steps to find posts that induce larger and larger normalized net flux. Note that $\left\{\tilde{x}_{j}\right\}$ are not equally-spaced along $\tilde{\gamma}$; a reparameterization is needed before starting a new iteration. 
Remark F.1. Since $\mathbf{y}_{i}$ is a discrete Fourier mode, we use $k_{i}$ to denote its wave number. To be more precise,

$$
\begin{aligned}
& k_{i}=i, \quad i \in\{1, \cdots, N / 2-1\}, \\
& k_{i}=i-N / 2, \quad i \in\{N / 2, \cdots, N\} .
\end{aligned}
$$

By definition, $\mathbf{y}_{i} \sim O(1)$. Then (F.1) implies $\partial_{s s}\left[V_{i} \cdot n\right] \sim O\left(k_{i}^{2}\right)$. By (C.26), it follows that $\kappa^{\prime}(x)$ corresponding to the perturbation vector field $V_{i}$ is of order $1+k_{i}^{2}$. Hence, $\mathcal{L}_{1}\left(\tilde{V}_{i} \cdot n\right)$ and $\mathcal{L}_{2}\left(V_{i} \cdot n\right)$ are going to be huge and highly oscillatory when $k_{i}$ is large, leading to very strong stiffness when solving for $\left(q_{i}^{\prime}, q_{B, i}^{+}{ }^{\prime}, q_{B, i}^{-}{ }^{\prime}\right)$. On the other hand, it also implies that $d E\left(\Omega ; \tilde{V}_{i}\right)$ is of order $1+k_{i}^{2}$, i.e. $E(\Omega)$ is sensitive to perturbations on $\gamma$ with higher frequencies. Combining this with (F.4), we see that the evolution of $\gamma$ in $(\mathrm{F} .5)$ will be mostly governed by the high-frequency modes, and thus the step-size has to be extremely small. Therefore, to remove the stiffness in the steepest ascent method (F.5), we make a modification of $\mathbf{G}$, denoted by $\mathbf{G}^{\prime}$.

Let $N^{\prime} \leq N / 2$ be a positive integer. Define

$$
\mathbf{G}^{\prime}=\sum_{\left\{i: k_{i} \leq N^{\prime}\right\}} \max \left\{1, k_{i}\right\}^{-2} \frac{d E\left(\Omega ; \tilde{V}_{i}\right)}{\left(\mathbf{y}_{i} \cdot \mathbf{y}_{i}\right) \Delta s} \mathbf{y}_{i},
$$

which also gives an ascent direction since $\mathbf{G} \cdot \mathbf{G}^{\prime}>0$. Then we replace $F .5$ by

$$
\tilde{x}_{j}=x_{j}+\varepsilon n\left(x_{j}\right) \cdot \mathbf{G}_{j}^{\prime} .
$$

In other words, high-frequency modes in $\mathbf{G}$ get suppressed or even filtered out. The coefficients $\max \left\{1, k_{i}\right\}^{-2}$ should be understood as preconditioning in the steepest ascent method. This enforces the smoothness of $V$, and also reduces computational costs if $N^{\prime} \ll N / 2$. 